UNIVERSITY OF ROCHESTER

LABORATORY FOR LASER ENERGETICS
Volume 60

July-September 1994

DOE/SF/19460-45
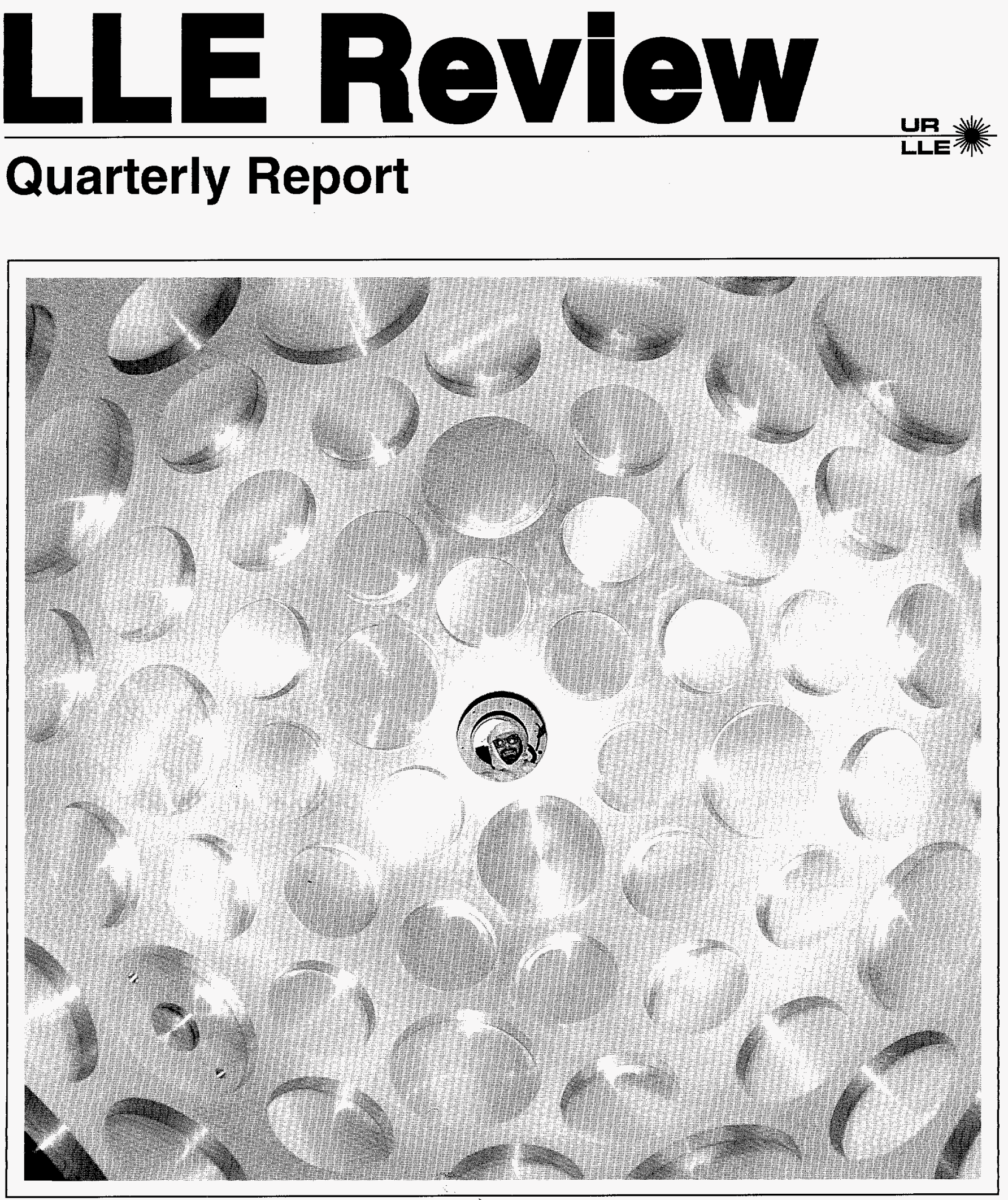
Printed in the United States of America Available from

National Technical Information Services U.S. Department of Commerce 5285 Port Royal Road Springfield, VA 22161

Price codes: Printed Copy A04 Microfiche A01

\section{About the Cover:}

Ken Walsh, an optical engineer in the Materials Sciences Group, is shown looking into the OMEGA Upgrade target chamber through one of the 60 beam ports. This photograph was taken with a wide-angle lens from the opposite beam port. A set of cross-hair fiducials are installed on each pair of 30 beam ports and the corresponding opposite port to define the optical axis for this pair of laser beams. A second set of cross hairs are then installed on the outer end of the hexagonal beam tubes aligned to the beam axis. The hexagonal-beam-tube cross hairs are used as an alignment fiducial for the installation and alignment of the turning mirrors and the targeting mirrors for each beam. The status of the OMEGA Upgrade is part of this LLE Review.

This report was prepared as an account of work conducted by the Laboratory for Laser Energetics and sponsored by New York State Energy Research and Development Authority, the University of Rochester, the U.S. Department of Energy, and other agencies. Neither the above named sponsors, nor any of their employees, makes any warranty, expressed or implied, or assumes any legal liability or responsibility for the accuracy, completeness, or usefulness of any information, apparatus, product, or process disclosed, or represents that its use would not infringe privately owned rights. Reference herein to any specific commercial product, process, or service by trade name, mark, manufacturer, or otherwise, does not necessarily constitute or imply its endorsement, recommendation, or favoring by the United States Government or any agency thereof or any other sponsor. Results reported in the LLE Review should not be taken as necessarily final results as they represent active research. The views and opinions of authors expressed herein do not necessarily state or reflect those of any of the above sponsoring entities.

The work described in this volume includes current research at the Laboratory for Laser Energetics, which is supported by New York State Research and Development Authority, the University of Rochester, the U.S. Department of Energy Office of Inertial Confinement Fusion under Cooperative Agreement No. DE-FC03-92SF19460, and other agencies.

For questions or comments, contact James P. Knauer, Editor, Laboratory for Laser Energetics, 250 East River Road, Rochester, NY 14623-1299, (716) 275-2074. 

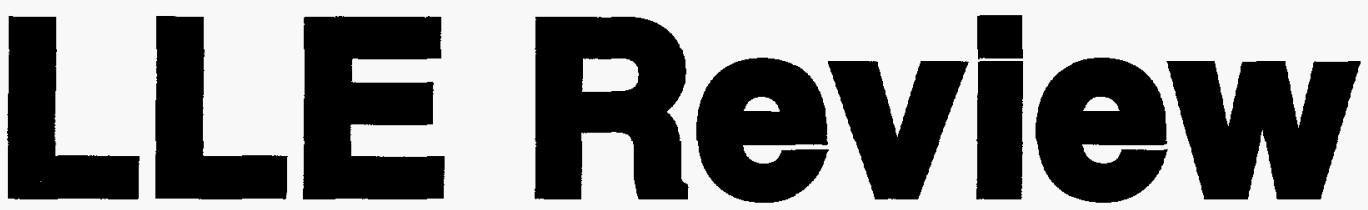

Quarterly Report

\section{Contents}

In Brief

Highly Efficient Second-Harmonic Generation

of Ultra-Intense Nd:Glass Laser Pulses

Multiple Cutoff Wave Numbers of the Ablative

Rayleigh-Taylor Instability

Ultrafast, All-Silicon Light Modulator

Angular Dependence of Stimulated Brillouin

Scattering in Homogeneous Plasma

Femtosecond Excited-State Dynamics of a

Conjugated Ladder Polymer

OMEGA Upgrade Status Report

10/1/93-9/30/94

NLUF News

Publications and Conference Presentations 



\section{DISCLAIMER}

This report was prepared as an account of work sponsored by an agency of the United States Government. Neither the United States Government nor any agency thereof, nor any of their employees, makes any wartanty, express or implied, or assumes any legal liability or responsibility for the accuracy, completeness, or usefulness of any information, apparatus, product, or process disclosed, or represents that its use would not infringe privately owned rights. Reference herein to any specific commercial product, process, or service by trade name, trademark, manufacturer, or otherwise does not necessarily constitute or imply its endorsement, recommendation, or favoring by the United States Government or any agency thereof. The views and opinions of authors expressed herein do not necessarily state or reflect those of the United States Government or any agency thereof. 


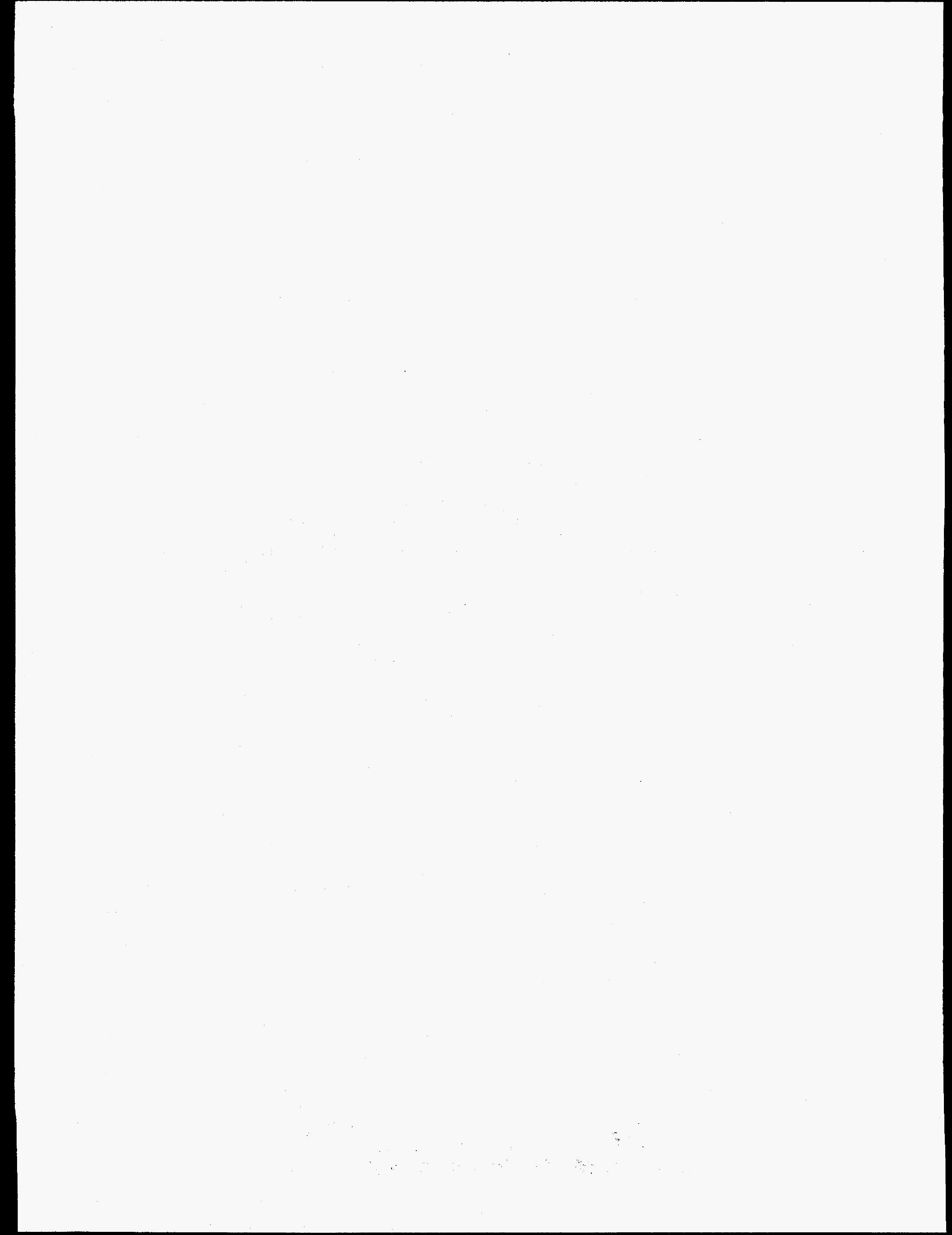




\section{DISCLAIMER}

Portions of this document may be illegible in electronic image products. Images are produced from the best available original document. 



\section{In Brief}

This volume of the LLE Review, covering the period of July-September 1994, contains articles on efficient generation of second-harmonic radiation from short-pulse lasers; calculation of the stabilization cutoff wave numbers for the Rayleigh-Taylor instability; a high-frequency silicon optical modulator; the angular dependence of stimulated Brillouin scattering; and femtosecond dynamics of ladder polymers. Three of these articles-second-harmonic generation, Rayleigh-Taylor cutoff wave numbers, and angular dependence of Brillouin scattering - are directly related to the OMEGA Upgrade, currently under construction. A summary of the status of the OMEGA Upgrade laser facility and the NLUF News for FY94 are included in this volume.

Highlights of the research presented in this issue are

- Second-harmonic conversion efficiency of $80 \%$ has been demonstrated with $0.5-p s, 1.053-\mu \mathrm{m}$ laser pulses. The experimental data are in generally good agreement with simulations and indicate that self-phase modulation and cross-phase modulation may be important.

- The cutoff wave number of the incompressible ablative Rayleigh-Taylor instability is calculated using the physical optics approximation of the WKB theory. A single value for the cutoff wave number can correspond to multiple modes with different eigenfunctions and growth rates.

- The carrier-refraction effect is used to modulate the refractive index of silicon. This should allow one to construct an all-silicon, light-intensity modulator for use as a transmitter of integratedcircuit optical communication. The bandwidth of the proposed modulator will be limited only by the RC time constant and is calculated to be about $40 \mathrm{GHz}$.

- The threshold of the absolute SBS instability and its steady-state spatial growth rate are independent of the scattering angle for homogenous plasmas of relevance to inertial confinement fusion. However, the saturation time of the convective instability does exhibit a strong inverse dependence on the scattering angle.

- Transient photoinduced bleaching of the $\pi-\pi^{*}$ transition accompanied with photoinduced absorption in the optical gap was observed for a thin-film conjugated-ladder polymer. Femtosecond time-resolved measurements of both a 2-eV (620-nm) optical pump and a whitelight-continuum probe show an absorption decay with a fast subpicosecond component and a slow, 20-ps component.

James P. Knauer

Editor 



\section{Highly Efficient Second-Harmonic Generation of Ultra-Intense Nd:Glass Laser Pulses}

Major advances in laser technology at LLE have included the demonstration of high-efficiency frequency doubling and tripling of fusion lasers ${ }^{1}$ and the development of short-pulse, chirped-pulse-amplification (CPA) lasers. ${ }^{2}$ These technologies were combined a few years ago with the demonstration of 75\% efficient frequency doubling of 1.6-ps laser pulses on the CPA T $^{3}$ (table-top terawatt) system at LLE, in collaboration with the Australian National University (ANU). ${ }^{3}$ Excellent agreement with simulations demonstrated that "standard" frequency conversion theory also applies in the picosecond regime, at intensities up to a few $\mathrm{GW} / \mathrm{cm}^{2}$.

This article reports a more recent collaboration with the University of Michigan. In an extensive series of experiments, 500-fs, 1053-nm laser pulses were converted to the second harmonic in KDP crystals at intensities up to $400 \mathrm{GW} / \mathrm{cm}^{2}$, with efficiencies of $\sim 80 \%$, without any obvious damage to the crystals. This result extends the regime of validity of the theory nearly two orders of magnitude higher in intensity. Further, the results provide evidence that, at these intensities, it is necessary to include self- and cross-phase modulation in the theory. Most significant, though, may be the extension of the available wavelength range of CPA systems used for ultra-intense lasermatter interaction experiments.

CPA lasers have been successfully used to generate peak laser powers in the infrared well beyond the terawatt regime. ${ }^{4-7}$ When focused, these lasers can produce intensities exceeding $10^{18} \mathrm{~W} / \mathrm{cm}^{2}$, giving access to new regimes of lasermatter interaction. For solid targets irradiated at these intensities the peak-to-background intensity contrast of the laser pulse should exceed $\sim 10^{9}: 1$ to avoid energy deposition in a plasma created by a much longer prepulse. Second-harmonic generation (SHG) in nonlinear crystals is thus important because, aside from extending the available wavelength range, it significantly improves the contrast ratio. Indeed, high-contrast, second-harmonic laser pulses have been used to produce high-density, high-temperature plasmas that emit short x-ray pulses in the keV region. ${ }^{8}$
The experiments reported here were carried out on a terawatt $\mathrm{Nd}$ :glass laser system. ${ }^{5}$ In these experiments the laser delivered energies up to $1.5 \mathrm{~J}$ with a wavelength of $1.053 \mu \mathrm{m}$ and a temporal full-width at half-maximum (FWHM) of $500 \mathrm{fs}$. The spatial profile was somewhat flatter than Gaussian with a diameter of $2.6 \mathrm{~cm}$ (FWHM). The intensity contrast ratio was $10^{6}: 1$, as measured with a third-order correlator. The pulse energies of the fundamental and its second harmonic were monitored by calorimeters. Three crystal configurations were examined: (a) a type-I crystal, optimum for the SHG of ultrashort $1-\mu \mathrm{m}$ pulses because the fundamental and secondharmonic group velocities are, for this wavelength, almost equal; (b) a type-II crystal, limited by temporal walkoff between the fundamental $o$ and $e$ pulses; and (c) a predelay scheme using two type-II crystals, ${ }^{9}$ in which compensation of this walkoff leads to improved efficiency, as was demonstrated in the collaborative experiments on $\mathrm{T}^{3}$ with the ANU, ${ }^{3}$ and second-harmonic pulse shortening. ${ }^{10}$ Results from the current experiments have included high conversion from the type-I crystal (70\%-80\% throughout the range of 100 to $400 \mathrm{GW} / \mathrm{cm}^{2}$ ) and evidence of pulse shortening (to $\sim 100-\mathrm{fs}$ FWHM) from the predelay scheme.

These experiments have been simulated with the LLE code $M I X E R,{ }^{11}$ enhanced to model group-velocity dispersion and $\chi^{(3)}$ effects. The code integrates equations of the form

$$
\begin{aligned}
\frac{\partial E_{3}}{\partial z}+\frac{1}{V_{g 3}} \frac{\partial E_{3}}{\partial t} & =-\frac{1}{2} \gamma_{3} E_{3}-i K_{3} E_{1} E_{2} \exp (i \Delta k \cdot z) \\
& +i\left\{\alpha_{31}\left|E_{1}^{2}\right|+\alpha_{32}\left|E_{2}^{2}\right|+\alpha_{33}\left|E_{3}^{2}\right|\right\} E_{3},
\end{aligned}
$$

where, for wave $i$ (waves 1 and 2 combining to generate wave 3 ), $E_{i}$ is the electric field, $V_{g i}$ is the group velocity, $\gamma_{i}$ is the linear absorption coefficient, $K_{i}$ is a nonlinear coefficient, and $\alpha_{i j}$ gives the change of refractive index due to the intensity of wave $j$ [resulting from the third-order susceptibility tensor 
$\left.\chi^{(3)}\right]$. The quantity $\Delta k$ is the wave-vector mismatch, $z$ indicates propagation distance, and $t$ denotes time. The coefficients $\alpha_{i j}$ are related to the more convenient coefficients $\gamma_{i j}$ defined via the equation $n_{i}=n_{i o}+\Sigma \gamma_{i j} I_{j}$, which gives the refractive index $n_{i}$ of wave $i$ in terms of its linear index $n_{i o}$ and a nonlinear correction summed over the intensities $I_{j}$ of waves $j$. For an isotropic medium and in the absence of dispersion, $\gamma_{i j}=\gamma_{0}$ for $i=j$ (self-phase modulation); for $i \neq j$ (cross-phase modulation), $\gamma_{i j}=2 \gamma_{0}$ if the polarizations of waves $i$ and $j$ are parallel and $2 \gamma_{0} / 3$ if they are perpendicular. ${ }^{12}$ The simulations have used $\gamma_{0}=0.27 \times 10^{-19} \mathrm{~m}^{2} / \mathrm{W}$ at $1 \mu \mathrm{m} .{ }^{13}$ Since no data is available for $0.5 \mu \mathrm{m}, M I X E R$ has used an ad hoc model where $\gamma_{i j}$ is multiplied by a dispersion factor $F \equiv 1.5$ if both waves are second harmonic and $F^{1 / 2}$ if one is. The propagation and $K_{3}$ terms of Eq. (1) are solved in the frequency domain and the $\chi^{(3)}$ term in the time domain. (In the frequency domain, the group-velocity term is replaced by the simple translation of each Fourier mode with its phase velocity, thus including all orders of dispersion.) Predictions for the experimental energyconversion efficiencies are obtained by averaging solutions to Eq. (1) over the measured two-dimensional spatial profile of the IR laser beam.

Results for the first experiment, doubling in a 4-mm, type-I crystal, are shown in Fig. 60.1(a). Conversion efficiencies of $70 \%-80 \%$ were found from 100 to $400 \mathrm{GW} / \mathrm{cm}^{2}$, with consistency between the two data sets (from different crystals used five months apart). The nominal intensity plotted on the horizontal axis is close to the intensity in the center of the beam at the peak of the pulse. The beam was modeled as a bandwidth-limited Gaussian of FWHM $500 \mathrm{fs}$, consistent with experimental spectra and autocorrelations.

Agreement with the simulation for both $\chi^{(3)}$ and $\Delta \theta_{1}=0$ (dashed curve) is generally reasonable, except for a falloff at high intensities $\left(\geq 100 \mathrm{GW} / \mathrm{cm}^{2}\right)$. One plausible explanation is nonlinear $\chi^{(3)}$-induced mismatch, ${ }^{14}$ modeled here with the solid curves for various angular detunings $\Delta \theta_{1}$ from smallsignal phase matching, measured outside the crystal, with positive $\Delta \theta_{1}$ indicating an increase in the angle between the propagation direction and the optic axis. The curve labeled $\Delta \theta_{1}=0$ would be followed if the small-signal tuning were sufficiently accurate to locate this direction. However, it was found necessary to fine tune the crystal at high intensity, and it is thus possible that one data set followed $\Delta \theta_{1} \approx 0.5-0.7 \mathrm{mrad}$ and the other $0.3 \mathrm{mrad}$. Dispersion in the (unknown) $\gamma_{i j}$ coefficients is critical to this explanation. Without dispersion $(F=1)$, the simulations do not depart significantly from the $\chi^{(3)}=0$ curve even with $\gamma_{0}$ five times bigger; moreover, with
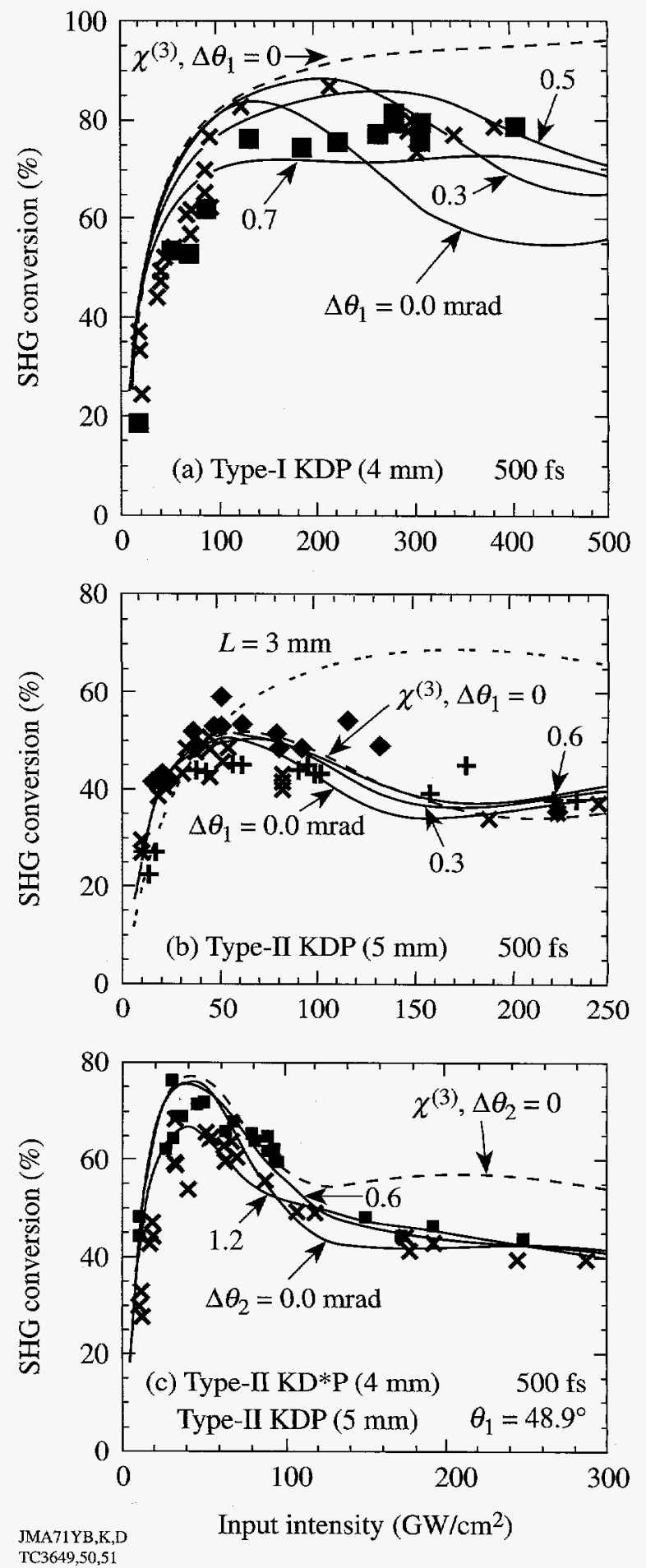

Figure 60.1

SHG efficiency as a function of input laser intensity for (a) a 4-mm, type-I KDP crystal; (b) a 5-mm, type-II KDP crystal; and (c) a 4-mm, detuned, typeII KD*P crystal followed by a 5-mm, type-II KDP crystal. Dashed curves: simulations for $\chi^{(3)}=0$ and no detuning. Solid curves: simulations including $\chi^{(3)}$ with various angle detunings (in mrad away from the optic axis). The double-dotted curve in (b) is for a 3-mm crystal with $\chi^{(3)}=0$. 
more dispersion $(F=2)$, the simulations fall below the data at $400 \mathrm{GW} / \mathrm{cm}^{2}$ for all $\Delta \theta_{1}$. It is worth noting that for any single intensity a $\Delta \theta_{1}$ can be chosen to fully compensate for the nonlinear phase error, ${ }^{15}$ but it is impossible to simultaneously compensate for the full range of intensities in the beam in space and time.

Results for a single, 5-mm-thick, type-II crystal are shown in Fig. 60.1(b) for three data sets. Conversion efficiencies reach $50 \%$ at $50 \mathrm{GW} / \mathrm{cm}^{2}$ and then fall off due to groupvelocity walkoff between the fundamental $o$ and $e$ waves. [This causes incorrect photon ratios (deviating from 1:1) leading to reconversion.] This crystal is much less sensitive to mismatch than the type-I crystal, and the inclusion of $\chi^{(3)}$ terms thus makes little difference. The reconversion seen above $60 \mathrm{GW} / \mathrm{cm}^{2}$ indicates that the crystal was too thick: the doubledotted curve shows that a 3-mm crystal would perform much better at the higher intensities. Thus, a type-I crystal has just a small advantage at $500 \mathrm{fs}$. For shorter pulses, however, it is strongly preferred: at $100 \mathrm{fs}$ and comparable intensities the predicted loss is insignificant for type I but catastrophic for type II.

In the third experiment the 5-mm, type-II KDP crystal was preceded by a 4-mm, type-II KD*P crystal, detuned several degrees from phase matching, that provided a predelay between the fundamental $o$ and $e$ rays. Since the sign of the detuning was unknown, it was assumed that the propagation angle within the $\mathrm{KD} * \mathrm{P}$ was $\theta_{1}=48.9^{\circ}$, giving a predelay of 340 fs. [Predictions for the other possibility $\left(\theta_{1}=58.3^{\circ}\right.$, predelay $=430 \mathrm{fs}$ ) are similar.] Results are shown in Fig. 60.1(c) for experiments carried out on two separate days. Within the data scatter, all curves provide a reasonable fit to the data below $\sim 100 \mathrm{GW} / \mathrm{cm}^{2}$. At higher intensities, however, the data fall significantly below the prediction for $\chi^{(3)}=0$ and lie much closer to the curves including $\chi^{(3)}$. As with the type-I case, there is an optimum positive detuning $\left(\Delta \theta_{2}\right)$ of the KDP crystal, although here the sensitivity to $\Delta \theta_{2}$ is smaller. The role of the $\chi^{(3)}$ terms here is more complicated since phase retardations generated at one time in the pulse affect phase matching at other times due to the various groupvelocity walkoffs.

The input polarization angle $\theta_{p}$ (the angle of the input electric field vector to the $o$ direction of the first crystal ${ }^{11}$ ) was controlled with a half-wave plate. Two conversion scans carried out as a function of $\theta_{p}$ are shown in Fig. 60.2, one for the single type-II crystal and one for the predelay scheme. Agreement with simulations is generally close, confirming

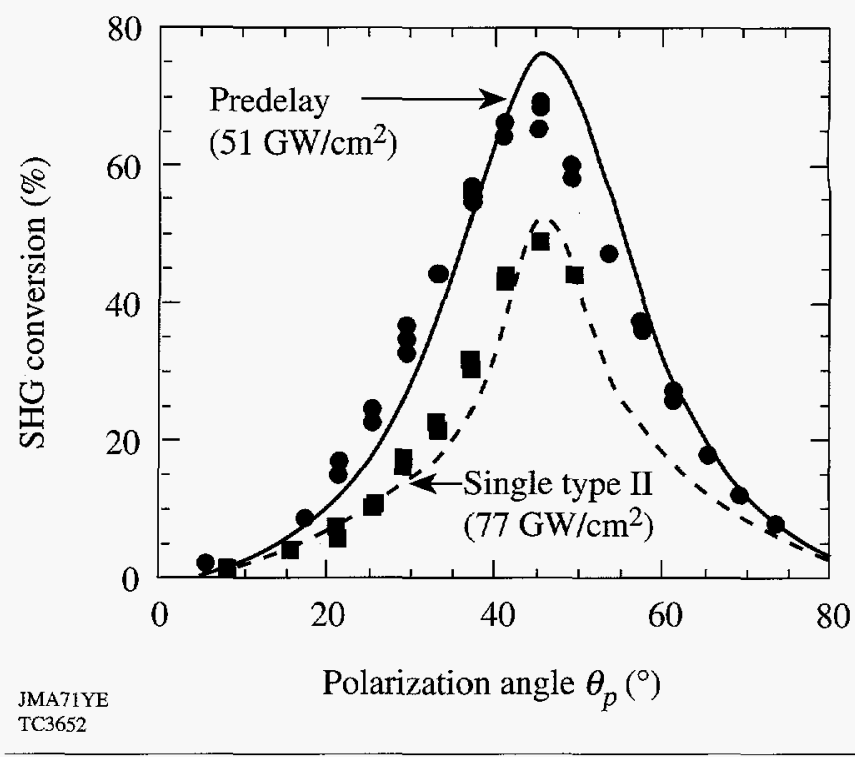

Figure 60.2

Dependence of the SHG efficiency on the polarization angle of the incident laser. Dashed curve: single, $5-\mathrm{mm}$, type-II KDP crystal at $77 \mathrm{GW} / \mathrm{cm}^{2}$. Solid curve: predelay scheme at $51 \mathrm{GW} / \mathrm{cm}^{2}$.

that the polarization state of the laser is substantially correct. The $10 \%$ discrepancy at the peak seen for the predelay scheme is consistent with the experimental scatter at the same intensity in Fig. 60.1(c).

The pulse shape of the second harmonic generated by the predelay scheme using a single-shot autocorrelator has been investigated. ${ }^{16}$ A portion of the second harmonic was split into two beams that were downcollimated and passed through a 100 - $\mu$ m-thick BBO crystal, incident at angles $\pm 10^{\circ}$ to the crystal normal and timed such that the autocorrelation sampled the center of the beam. An experimental fourth-harmonic trace is shown in Fig. 60.3 (a) for an estimated IR intensity of $119 \mathrm{GW} / \mathrm{cm}^{2}$ in the sampled region together with a prediction, including the same $\chi^{(3)}$ model used in Fig. 60.1, for $\Delta \theta_{2}=$ $1.8 \mathrm{mrad}$ (the optimum detuning at this intensity). The match is very close, except in the wings where the asymmetry is probably explained by spatial intensity variations in the beam. The predicted second-harmonic pulse shape generated by the KDP crystal [Fig. 60.3(b)] shows a narrow central spike with an intensity FWHM of $84 \mathrm{fs}$, superposed upon a broader wing at the $\sim 15 \%$ intensity level. [With $\chi^{(3)}=0$, a 49 -fs FWHM is predicted.] The autocorrelation calculation included propagation through $1.5 \mathrm{~cm}$ of BK7 glass (which significantly broadened the pulse to 166-fs FWHM) and group-velocity dispersion within the BBO. It should be cautioned that the $1.8 \mathrm{-mrad}$ detuning was not measured but was chosen to illustrate a possible temporal pulse shape consistent with the measured 


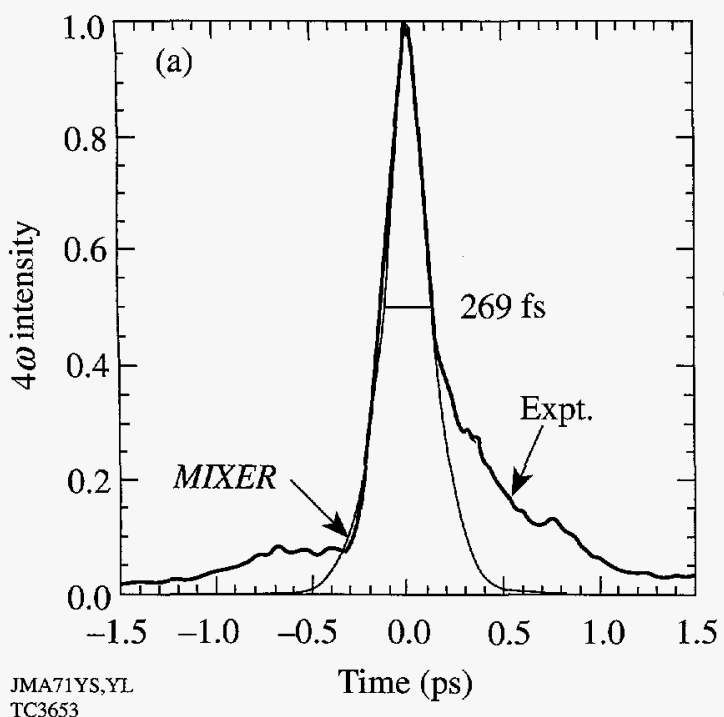

autocorrelation. Moreover, above $\sim 100 \mathrm{GW} / \mathrm{cm}^{2}$, structure was generated in the second-harmonic beam that limited its focusability, and the second-harmonic spectrum was broader than predicted with the $\chi^{(3)}$ model. The first demonstration of pulse shortening using the predelay scheme ${ }^{10}(1.2$ ps to $250 \mathrm{fs}$ at $7 \mathrm{GW} / \mathrm{cm}^{2}$ ) was unlikely to have been affected by $\chi^{(3)}$ terms.

In conclusion, an extensive series of SHG experiments have been conducted using intense, 500-fs laser pulses. Efficiencies of $70 \%-80 \%$ up to $400 \mathrm{GW} / \mathrm{cm}^{2}$ were obtained from a type-I crystal without any obvious damage to the crystal or degradation of focusability. Lower efficiencies were obtained for a type-II crystal. The results for the predelay scheme were consistent with a pulse shortening to $\$ 100$-fs FWHM, although with low contrast. All results are broadly in agreement with predictions of the code $M I X E R$, with improved agreement obtained above $\sim 100 \mathrm{GW} / \mathrm{cm}^{2}$ by including a $\chi^{(3)}$ model that makes $a d$ hoc assumptions about the dispersion of the self- and cross-phase-modulation coefficients. Further experiments are needed to measure these unknown coefficients. The most important practical consequence of the experiments reported here may be the extension of ultra-intense laser-matter experiments to shorter wavelengths.

\section{ACKNOWLEDGMENT}

These experiments were carried out at the University of Michigan by Drs. C. Y. Chien, G. Korn, J. S. Coe, J. Squier, and G. Mourou. This work was supported by the National Science Foundation through the Center for Ultrafast Optical Science under STC PHY 8920108 and by the U.S. Department of Energy Office of Inertial Confinement Fusion under Cooperative Agreement No. DE-FC03-92SF19460, the University of Rochester, and the New York State Energy Research and Development Authority. The support of DOE does not constitute an endorsement by DOE of the views expressed in this article.

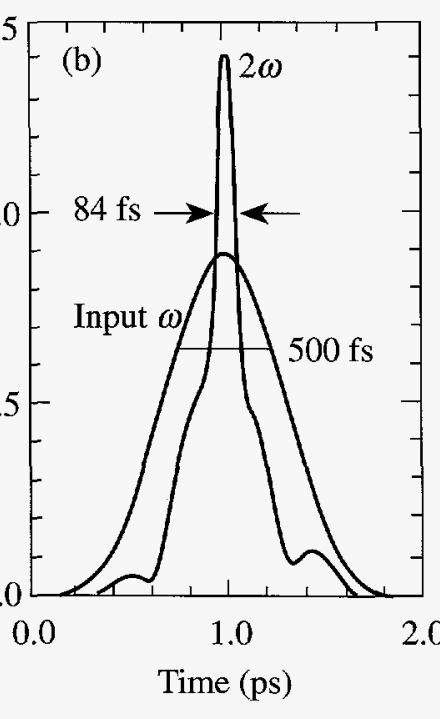

Figure 60.3

(a) Experimental and simulated second-harmonic autocorrelation intensity for the predelay scheme at $119 \mathrm{GW} / \mathrm{cm}^{2}$. The simulation includes $\chi^{(3)}$ effects and assumes a detuning of $1.8 \mathrm{mrad}$. (b) Simulated secondharmonic pulse shape at the output of the KDP crystal (electric field versus time).

\section{REFERENCES}

1. W. Seka, S. D. Jacobs, J. E. Rizzo, R. Boni, and R. S. Craxton, Opt. Commun. 34, 469 (1980).

2. D. Strickland and G. Mourou, Opt. Commun. 56, 219 (1985).

3. Y. Wang, B. Luther-Davies, Y.-H. Chuang, R. S. Craxton, and D. D. Meyerhofer, Opt. Lett. 16, 1862 (1991).

4. F. G. Patterson, R. Gonzales, and M. D. Perry, Opt. Lett. 16, 1107 (1991).

5. Y. Beaudoin et al., Opt. Lett. 17, 865 (1992).

6. G. Rouyer et al., Opt. Lett. 18, 214 (1993).

7. C. N. Danson et al., Opt. Commun. 103, 392 (1993).

8. C. Y. Chien et al., Opt. Lett. 18, 1535 (1993).

9. Y. Wang and R. Dragila, Phys. Rev. A 41, 5645 (1990).

10. Y. Wang and B. Luther-Davies, Opt. Lett. 17, 1459 (1992).

11. R. S. Craxton, IEEE J. Quantum Electron. QE-17, 1771 (1981).

12. G. P. Agrawal, Nonlinear Fiber Optics (Academic Press, San Diego, 1989).

13. D. Milam and M. Weber, Opt. Commun. 18, 172 (1976).

14. L. S. Telegin and A. S. Chirkin, Sov. J. Quantum Electron. 12, 1354 (1982).

15. C. J. McKinstrie and X. D. Cao, J. Opt. Soc. Am. B 10, 898 (1993).

16. F. Salin, P. Georges, G. Roger, and A. Brun, Appt. Opt. 26, 4528 (1987). 


\section{Multiple Cutoff Wave Numbers of the Ablative Rayleigh-Taylor Instability}

The Rayleigh-Taylor instability occurs at the interface between heavy and light fluids when the heavy fluid is accelerated by the light fluid. The classical treatment of a sharp interface shows that a small perturbation at this boundary will grow as $e^{\gamma t}$, where $\gamma$ is the linear growth. ${ }^{1}$ The ablation front of an inertial confinement fusion (ICF) imploding target is subject to this instability because the compressed target is accelerated by the low-density ablating plasma. If small perturbations caused by either target imperfections or illumination nonuniformity grew classically, then these small perturbations would grow to sufficient amplitudes to destroy the shell of the target and degrade the performance of the implosion. It has recently been shown by several authors ${ }^{2-9}$ that the ablation process will actually reduce the Rayleigh-Taylor growth rate at this interface and can, in fact, stabilize the interface at sufficiently short wavelengths. Calculations of the cutoff wave number for a diffuse density profile were carried out by $\mathrm{Kull}^{7}$ and by Bud'ko and Liberman. ${ }^{8}$ Using the assumption that the cutoff occurs at wavelengths shorter that the density-gradient scale length $L=[(1 / \rho)(d \rho / d y)]^{-1}$, Bud'ko and Liberman ${ }^{8}$ used the geometrical optics approximation of the Wentzel-KramersBrillouin (WKB) theory to derive the cutoff wave number in the limit of $V_{a} / \sqrt{g L} \rightarrow 0$, where $V_{a}$ is the ablation velocity of the overdense portion of the target.

The role of the parameter $\Sigma=V_{a} / \sqrt{g L}$ can be easily deduced by using the following intuitive form of the instability growth rate, $\gamma \sim \sqrt{k g /(1+k L)}-k V_{a}$. By setting $\gamma=0$, it is easy to show that for $\Sigma \gg 1$ the cutoff wave number occurs at wavelengths longer than the density-gradient scale length $\left(k_{c} L \sim 1 / \Sigma^{2} \ll 1\right)$. On the contrary, for $\Sigma \ll<$, the cutoff occurs a wavelength shorter than $L\left(k_{c} L \sim 1 / \Sigma \gg 1\right)$. The relative size of the cutoff wavelength to the density-gradient scale length suggests the type of mathematical technique that must be used. It is well known that short-wavelength modes with $k_{c} L \gg>1$ can be investigated using the WKB approximation, and long-wavelength modes $\left(k_{c} L \ll 1\right)$ have a characteristic "boundary layer" structure in the sharp gradient region and can be studied with a sharp boundary model.
In this article, we derive the physical optics approximation of the WKB theory applied to the ablative Rayleigh-Taylor instability for $\Sigma \ll<1$, and we show the existence of multiple branches in the instability spectrum. Each branch has a different cutoff wave number and an eigenfunction characterized by a different number of zeros. Furthermore, since in typical ICF targets the density profiles are rather steep (direct drive) or the ablation velocity is rather large (indirect drive), the parameter $V_{a} / \sqrt{g L}$ is only approximately less than 1 . The physical optics approximation also provides the next-order correction (in $\left.V_{a} / \sqrt{g L}<1\right)$ to the largest cutoff wave number.

\section{The WKB Approximation}

We consider an equilibrium in the frame of reference of the ablation front with the heavy fluid of density $\rho_{h}$ moving with velocity $U_{h}=-V_{a} \mathbf{e}_{y}$ (Fig. 60.4). The density smoothly varies from $\rho_{h}$ to a lower value $\rho_{l}$, and the velocity increases according to the conservation of mass flow ( $\rho U=$ constant). The fluid is subject to a force field $\mathbf{g}=g \mathbf{e}_{y}$ opposite to the density gradient $(g<0)$, and the density profile has a finite densitygradient scale length in the ablation region $(y \approx 0)$ with characteristic value $L_{0}\left[L(0) / L_{0} \sim 1\right]$. To treat the linear stability of ablation fronts, we consider a simplified incompressible model for the perturbation that is valid for subsonic ablation flow $\left(V_{a}\right.$ $\ll C_{s}$, where $C_{s}$ is the sound speed)

$$
\begin{aligned}
& \frac{d \rho}{d t}+\rho \nabla \cdot \mathbf{v}=0 \\
& \rho \frac{d \mathbf{v}}{d t}=-\nabla p+\rho \mathbf{g} \\
& \nabla \cdot(\mathbf{v}-\mathbf{U})=0
\end{aligned}
$$

where $\mathbf{U}$ is the equilibrium velocity and $d / d t=\partial / \partial t+\mathbf{v} \cdot \nabla$.

The set of linearized conservation equations can be written in the following form: 


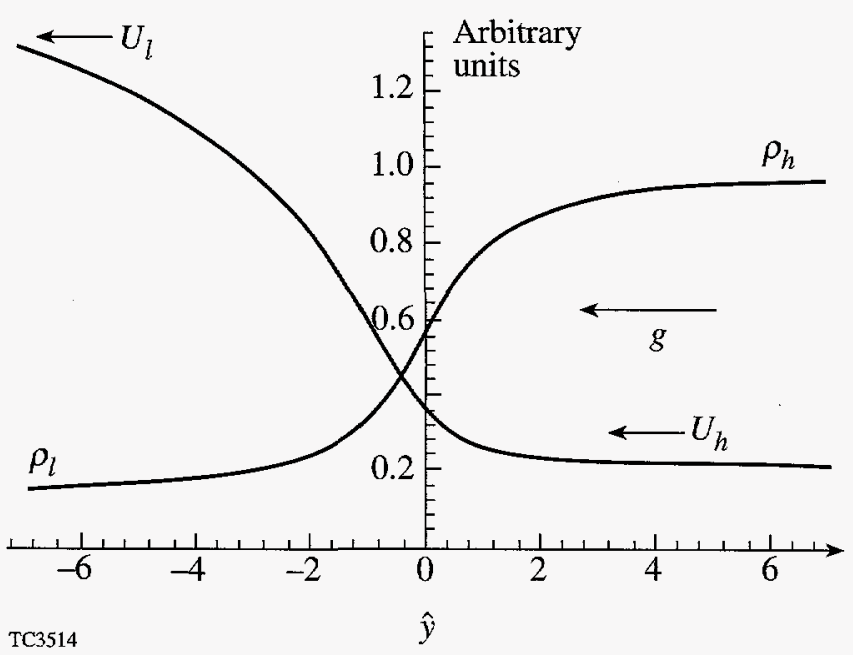

Figure 60.4

Density and velocity profiles. The subscripts $l$ and $h$ indicate the light and heavy fluid respectively.

$$
\begin{gathered}
\left(\frac{\Gamma}{\epsilon}+\partial_{\hat{y}}\right) \tilde{n}=-\tilde{v}_{y} / U \hat{L} \\
\left(\frac{\Gamma}{\epsilon}+\partial_{\hat{y}}\right) \tilde{v}_{x}=-i \tilde{p} / \epsilon \rho U \\
\left(\frac{\Gamma}{\epsilon}+\partial_{\hat{y}}-1 / \hat{L}\right) \tilde{v}_{y}=-\partial_{\hat{y}}(\tilde{p} / \rho U)-\tilde{n} G L_{0} / U \\
\partial_{\hat{y}} \tilde{v}_{y}=-i \tilde{v}_{x} / \epsilon,
\end{gathered}
$$

where $\epsilon=1 /\left(k L_{0}\right), \hat{y}=y / L_{0}, \tilde{n}=\tilde{\rho} / \rho, G=-g-U^{2} / L$, $\hat{L}=L / L_{0}$, and $\Gamma=\gamma / k U$. Equations (2)-(4) can be combined into a single fourth-order ordinary differential equation

$$
\begin{aligned}
& \left(\frac{\Gamma}{\epsilon}+\partial_{\hat{y}}-\alpha / \hat{L}\right) \\
& {\left[\epsilon^{4} \partial_{\hat{y}}\left(\frac{\Gamma}{\epsilon}+\partial_{\hat{y}}\right) \partial_{\hat{y}} \tilde{v}_{y}-\epsilon^{2}\left(\frac{\Gamma}{\epsilon}+\partial_{\hat{y}}-1 / \hat{L}\right) \tilde{v}_{y}\right]} \\
& +\tilde{z}_{y} \sigma^{2} / \hat{L}=0,
\end{aligned}
$$

where $\alpha=-\hat{L} \partial_{\hat{y}} \ln (U / G)$ and $\sigma^{2}=G / k^{2} U^{2} L_{0}$. Since Eq. (5) cannot be solved exactly, we look for an approximate solution when the parameter $\Sigma^{2}=V_{a}^{2} / g L_{0}$ is much less than unity, and we order $\epsilon \sim \Sigma<<1, \sigma \sim 1, G \simeq-g\left[1+O\left(\epsilon^{2}\right)\right]$, and $\alpha \simeq 1+O\left(\epsilon^{2}\right)$. The validity of the chosen ordering will be verified a posteriori. We apply the WKB theory to the fourthorder equation, and we adopt the following ansatz for the perturbation: $\tilde{v}_{y}=A(\hat{y}) \exp [S(\hat{y}) / \epsilon]$, where $A(\hat{y})$ and $S(\hat{y})$ are two slowly varying functions of $\hat{y}$, i.e., $\partial_{\hat{y}} \ln S \sim \partial_{\hat{y}} \ln A \sim 1$. The equation for $S(\hat{y})$ (geometrical optics) can be easily derived by retaining the lowest-order terms $(\sim 1)$ in Eq. (5):

$$
\left(S^{\prime}-\Gamma\right)^{2}\left(S^{\prime 2}-1\right)+\sigma^{2} / \hat{L}=0
$$

This equation is identical to the one derived in Ref. 8. By focusing on the mode corresponding to the cutoff wave number $k_{c}\left[\operatorname{Lim}_{k \rightarrow k_{c}} \gamma(k) \rightarrow 0^{+}\right]$, we solve Eq. (6) for $|\Gamma| \ll 1$ and find the four roots

$$
\begin{aligned}
& \left.S_{1}=(1 / \sqrt{2})\right]_{\frac{y}{y}}^{\hat{y}}\left[\left(q^{+}\right)^{1 / 2}-\tau^{-}\right] d \hat{y} \\
& S_{2}=-(1 / \sqrt{2}) \int_{\bar{y}}^{\hat{y}}\left[\left(q^{+}\right)^{1 / 2}+\tau^{-}\right] d \hat{y} \\
& S_{3}=(1 / \sqrt{2}) \int_{\bar{y}}^{\hat{y}}\left[\left(q^{-}\right)^{1 / 2}-\tau^{+}\right] d \hat{y} \\
& S_{4}=-(1 / \sqrt{2}) \int_{\frac{y}{y}}^{\hat{y}}\left[\left(q^{-}\right)^{1 / 2}+\tau^{+}\right] d \hat{y},
\end{aligned}
$$

where

$$
q^{ \pm}=1 \pm 2 \sqrt{Q}, \quad \tau^{ \pm}=\frac{\Gamma}{\sqrt{2}}\left(1 \pm \frac{1}{2 \sqrt{Q}}\right)
$$

$Q(\hat{y}) \equiv 1 / 4-\sigma^{2} / \hat{L}$, and $\bar{y}$ is an arbitrary point. Observe that Eqs. (7)-(8) are valid for nonvanishing $Q$ and the small $t$ corrections are important only for $|\hat{y}| \rightarrow \pm \infty$, where $q^{-} \rightarrow 0$, and they can be neglected for any other value of $\hat{y}$. If $Q$ vanishes at some point, Eq. (6) can be easily solved in the neighborhood of that point and $\Gamma \rightarrow 0^{-}$yielding $S \cong \pm(\hat{y}-\bar{y}) / \sqrt{2}$. This result can also be recovered from Eqs. (7)-(8) by neglecting $\tau$ even for $Q \approx 0$. We emphasize that the parameter $\tau$ is important for $S_{3}$ and $S_{4}$ only when $|\hat{y}| \rightarrow \infty$. While in Ref. 8 the analysis is limited to the geometrical optics [Eqs.(6)-(8)], here we extend the solution to include the physical optics approximation. By retaining the $\in$ corrections 
in Eq. (6), the following expressions for $A(\hat{y})$ are derived:

$$
\begin{gathered}
A_{1}=a_{1} F^{+}(\hat{y}) \\
A_{2}=a_{2} F^{+}(\hat{y}) \\
A_{3}=a_{3} F^{-}(\hat{y}) \\
A_{4}=a_{4} F^{-}(\hat{y}) \\
F^{ \pm}(\hat{y})=\left(\frac{G}{|U|}\right)^{1 / 4} \frac{1}{Q^{1 / 4}(1 \pm 2 \sqrt{Q})^{1 / 4}} \exp \left[\mathrm{m} \frac{3}{8} \int_{\hat{y}}^{\hat{y}} \frac{d \hat{y}}{\hat{L} \sqrt{Q}}\right] .
\end{gathered}
$$

It is important to recognize that none of the eigenfunctions represented by Eqs. (7)-(10) satisfies the boundary conditions of vanishing amplitude at both $+\infty$ and $-\infty$ simultaneously. This observation is supported by the form of the exponential terms in $S_{j}$ and $A_{j}$ and by the asymptotic behavior of $Q(\hat{y})$ [Fig. 60.5(a)]. It is readily derived from Eqs. (7)-(10) that in order to satisfy the boundary conditions at $y \rightarrow+\infty$, the coefficients $a_{1}, a_{3}, a_{4}$ must vanish $\left(a_{1}=a_{3}=a_{4}=0\right.$ and $a_{2} \neq 0$ ). On the contrary, to satisfy the boundary condition at $y \rightarrow-\infty$, the coefficient $a_{2}$ must vanish $\left(a_{2}=0\right)$. Thus, the solution valid for positive $y$ must be matched to a different

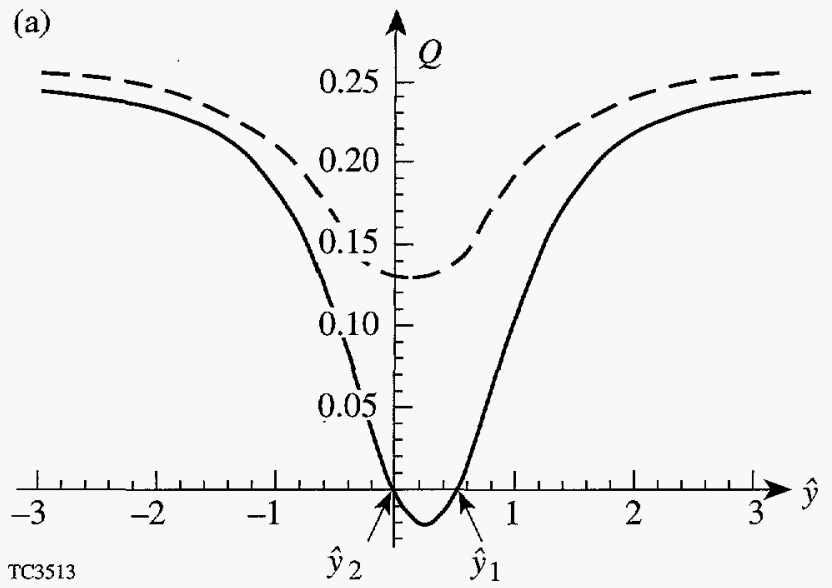

solution valid for negative $y$. The necessary condition for the matching to occur is that $Q(\hat{y})$ vanishes at some point and the $A_{j}$ 's become singular. The solid line of Fig. 60.5(a) represents a possible behavior of $Q(\hat{y})$ that would allow the matching. In general there must be two points (turning points) where $Q$ vanishes $\left(\hat{y}=\hat{y}_{1}\right.$ and $\hat{y}=\hat{y}_{2}$ with $\left.\hat{y}_{1}>\hat{y}_{2}\right)$ and at such points, the WKB approximation breaks down, i.e., the $A_{j}$ 's become singular. By defining $\hat{y}_{0}$ the point of minimum of

$$
Q^{\prime}\left(\hat{y}_{0}\right)=0, Q\left(\hat{y}_{0}\right)<0, Q^{\prime \prime}\left(\hat{y}_{0}\right)>0,
$$

we order $Q\left(\hat{y}_{0}\right) \sim \in$ and $\hat{y}_{1}-\hat{y}_{2} \sim \sqrt{\epsilon}$. This ordering is verified later by the matching conditions of the solution between the turning points with the WKB approximations. As shown in Fig. 60.5(b), three regions can be identified: (1) the first outer region for $\hat{y}>\hat{y}_{1}$, where

$$
\tilde{v}_{y}=A_{2}(\hat{y}) \exp \left[S_{2}(\hat{y}) / \epsilon\right]
$$

(2) the second outer region for $\hat{y}<\hat{y}_{2}$, where

$$
\tilde{v}_{y}=A_{1} \exp \left[S_{1} / \epsilon\right]+A_{3} \exp \left[S_{3} / \epsilon\right]+A_{4} \exp \left[S_{4} / \epsilon\right]
$$

and (3) the inner region between the turning points $\hat{y}_{2}<\hat{y}<\hat{y}_{1}$. To determine the solution in the inner region, we look at the behavior of the solution in the first outer region for $\sqrt{\epsilon} \ll \hat{y}-\hat{y}_{0} \ll<1$ and approximate $Q$ with its Taylor expansion $Q \approx Q_{0}+Q_{0}^{\prime \prime}\left(\hat{y}-\hat{y}_{0}\right)^{2} / 2$. By setting $\bar{y}=\hat{y}_{0}$, a straightforward manipulation yields

(b)

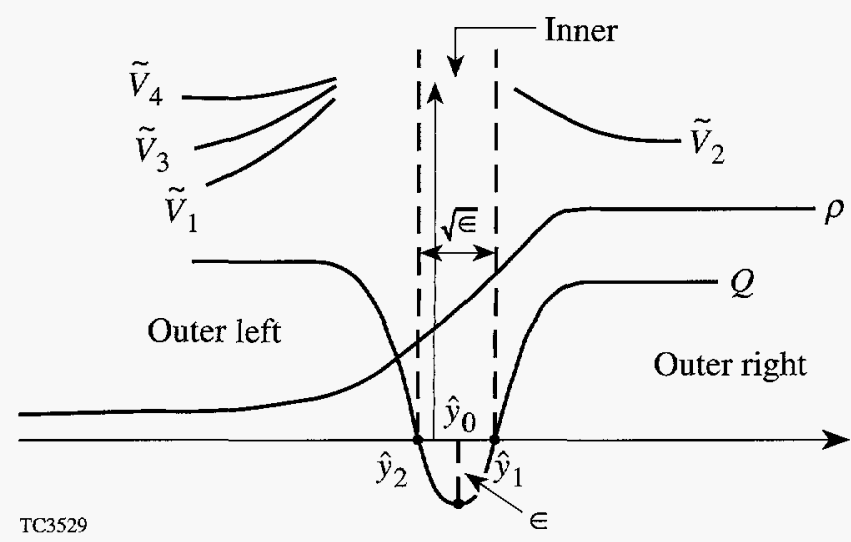

Figure 60.5

(a) Plot of $Q$ versus $\hat{y}$. The dashed line represents a behavior without zeros $\left[\left(\sigma^{2} / \hat{L}\right)_{\max }<1 / 4\right]$. The solid line shows a $Q(\hat{y})$ with two zeros $\left[\left(\sigma^{2} / \hat{L}\right)_{\max }>1 / 4\right]$. For large $|\hat{y}|, Q$ approaches $1 / 4$. (b) Plot of the WKB solutions in the outer regions $\hat{y} \gg \hat{y}_{1}$ and $\hat{y} \ll \hat{y}_{2}$. 


$$
\tilde{v}_{y}\left(\hat{y} \approx \hat{y}_{0}^{-}\right)=\hat{a}_{2} \hat{x}^{v} \exp \left[-\frac{1}{4} \sqrt{Q_{0}^{\prime \prime}} \hat{x}^{2}\right] \exp \left[-\frac{\left(\hat{y}-\hat{y}_{0}\right)}{\sqrt{2} \in}\right],
$$

where $\hat{x}=\left(\hat{y}-\hat{y}_{0}\right) / \sqrt{\epsilon}$, and

$$
\begin{aligned}
& v=-\frac{1}{\sqrt{Q_{0}^{\prime \prime}}}\left(\frac{3}{2^{5 / 2} \hat{L}\left(\hat{y}_{0}\right)}\right)-\frac{1}{2}-\frac{Q_{0}}{\sqrt{Q_{0}^{\prime \prime}}} \frac{1}{2 \epsilon} \\
& \hat{a}_{2}=a_{2} \frac{\sqrt{2}}{Q_{0}^{1 / 4}}\left[\frac{G\left(\hat{y}_{0}\right)}{\left|U\left(\hat{y}_{0}\right)\right|}\right]^{1 / 4}\left(\frac{2 \in Q_{0}^{\prime \prime}}{Q_{0}}\right)^{v / 2} .
\end{aligned}
$$

The function given by Eq. (11) shows a two-scale structure (the two scales being $\in$ and $\sqrt{\epsilon}$ ), different from what is found from the familiar second-order WKB solution near the turning points. Thus, in order to perform the matching, the solution between the turning points must retain the two-scale structure and behave as $\tilde{z}_{y}=\tilde{u}(\hat{x}) \exp \left[-\left(\hat{y}-\hat{y}_{0}\right) / \sqrt{2} \in\right]$. Substituting into the general Eq. (6) and retaining the lowest-order terms in $\epsilon$ leads to the following equation for $\tilde{u}$ :

$$
\left[\partial_{\xi}^{2}+v+\frac{1}{2}-\frac{1}{4} \xi^{2}\right] \tilde{u}=0,
$$

where $\xi=\hat{x}\left(Q_{0}^{\prime \prime}\right)^{1 / 4}$. The equation for $\tilde{u}$ is just a second-order equation (instead of fourth order) and can be exactly solved. The solution of Eq. (13) is the combination of two parabolic cylinder functions $\tilde{u}=B D_{v}(\xi)+C D_{v}(-\xi)$. Matching the inner with the outer solution for $\hat{y}>\hat{y}_{1}$ leads to $C=0$ and $B=\hat{a}_{2} /\left(Q_{0}^{\prime \prime}\right)^{v / 4}$. To match the rapidly varying exponential $\left\{\exp \left[-\left(\hat{y}-\hat{y}_{0}\right) / \sqrt{2} \epsilon\right]\right\}$ of the inner solution with the outer solution in the region $\hat{y}<\hat{y}_{2}$, the coefficients $a_{1}$ and $a_{3}$ of the outer solution must vanish. Therefore, as $\hat{y}$ approaches $\hat{y}_{2}$ $\left(\sqrt{\epsilon} \ll<\hat{y}_{0}-\hat{y} \ll<1\right)$, the outer solution assumes the following form:

$$
\tilde{v}_{y}\left(\hat{y} \approx \hat{y}_{0}^{+}\right)=\hat{a}_{4}(-\hat{x})^{v} \exp \left[-\frac{1}{4} \sqrt{Q_{0}^{\prime \prime}} \hat{x}^{2}\right] \exp \left[-\frac{\left(\hat{y}-\hat{y}_{0}\right)}{\sqrt{2} \in}\right],
$$

where

$$
\hat{a}_{4}=a_{4} \frac{\sqrt{2}}{Q_{0}^{1 / 4}}\left[\frac{G\left(\hat{y}_{0}\right)}{\left|U\left(\hat{y}_{0}\right)\right|}\right]^{1 / 4}\left(\frac{2 \in Q_{0}^{\prime \prime}}{Q_{0}}\right)^{v / 2} .
$$

Focusing on the inner solution, the asymptotic behavior of the parabolic cylinder function is easily derived:

$$
D_{v}(\xi \rightarrow-\infty) \sim \xi^{-v-1} \exp \left[\xi^{2} / 4\right] \quad v \neq n
$$

$$
D_{n}(\xi \rightarrow-\infty) \sim \xi^{n} \exp \left[-\xi^{2} / 4\right] \quad v=n,
$$

where $n$ is an integer. By matching the inner solution with Eq. (15), we immediately deduce that $v$ must be an integer $(v=n$ with $n=0,1,2 \ldots), a_{4}=(-1)^{n} a_{2}$, and

$$
D_{n}(\xi)=2^{-n / 2} \exp \left[-\xi^{2} / 4\right] H_{n}[\xi / \sqrt{2}]
$$

( $H_{n}$ is the Hermite polynomial). The condition $v=n$ represents the equation for the cutoff wave number and can be rewritten in the extended form

$$
\frac{Q_{0}}{\sqrt{Q_{0}^{\prime \prime}}} \frac{1}{2 \epsilon}=-\left(n+\frac{1}{2}\right)-\frac{1}{4 \sqrt{2 Q_{0}^{\prime \prime}}} \frac{3}{\hat{L}\left(\hat{y}_{0}\right)}
$$

where $\epsilon=1 /\left(k_{c} L_{0}\right)$. Observe that Eq. (18) yields $Q_{0} \sim \in$ and $\sigma \sim 1$, in agreement with the initial assumptions. Equation (18) can be solved perturbatively by expanding $k_{c}$ in powers of $\Sigma<1\left(k_{c}=k_{0}-\Sigma k_{1} \ldots\right)$. A short calculation yields the following expression for the cutoff wave number

$k_{c}=k_{0}\left\{1-\beta \Sigma\left[(2 n+1) \sqrt{\frac{d^{2} Q}{d \hat{y}^{2}}\left(\hat{y}_{0}\right)}+\frac{3}{2^{3 / 2}} \frac{1}{\hat{L}\left(\hat{y}_{0}\right)}\right]\right\}$,

where $k_{0}=2\left[|g| / L\left(\hat{y}_{0}\right)\right]^{1 / 2} /\left|U\left(\hat{y}_{0}\right)\right|, \quad \beta=\sqrt{\hat{L}\left(\hat{y}_{0}\right)} \rho_{h} / \rho\left(\hat{y}_{0}\right)$, and $n=0,1,2 \ldots$.

\section{Discussion}

The first important result of Eq. (19) is that multiple cutoff wave numbers exist for different values of $n$. In the $\gamma, k$ plane, this leads to an unstable spectrum characterized by multiple branches lying one below the other. The branch with the largest cutoff and therefore the largest growth rate is for $n=0$. Although the lowest-order cutoff wave number $k_{0}$ was previously found in Ref. 8, we emphasize the importance of the first-order correction to determine the existence of the multiple branches and to provide a more accurate formula for the $n=0$ branch when $\Sigma \leqslant 1$. Table 60.I provides a comparison 
between the cutoff wave number of the $n=0$ branch predicted by Eq. (19) and the one of Ref. 8 for the following form of the density profile

$$
\rho(y)=\frac{\rho_{h}}{1+A}\left[1+\frac{2}{\pi} A \tan ^{-1} \frac{y}{L_{0}}\right]
$$

Table 60.I Cutoff wave number for $n=0$. Comparison of the cutoff wave number for the $n=$ 0 branch predicted by Eq. (19) and Ref. 8 for $A=0.9, g=5 \times 10^{15} \mathrm{~cm} / \mathrm{s}^{2}, v_{\mathrm{a}}=7.5 \times$ $10^{4} \mathrm{~cm} / \mathrm{s}$, and varying $L_{0}$.

\begin{tabular}{|c|c|c|c|}
\hline$L_{0}(\mu \mathrm{m})$ & $\begin{array}{c}\text { Eq. (19) } \\
\left(\mu \mathrm{m}^{-1}\right)\end{array}$ & $\begin{array}{c}\text { Ref. 8 } \\
\left(\mu \mathrm{m}^{-1}\right)\end{array}$ & $\Sigma$ \\
\hline 50.0 & 1.05 & 1.10 & 0.015 \\
\hline 10.0 & 2.22 & 2.66 & 0.034 \\
\hline 5.0 & 2.99 & 3.48 & 0.047 \\
\hline 3.0 & 3.68 & 4.49 & 0.061 \\
\hline 2.0 & 4.28 & 5.50 & 0.075 \\
\hline 1.0 & 5.33 & 7.78 & 0.11 \\
\hline 0.5 & 6.10 & 11.01 & 0.15 \\
\hline
\end{tabular}

Observe that the first-order correction in $\Sigma$ becomes important when the density profile is rather steep (direct-drive ICF) or the ablation velocity is rather large (indirect-drive ICF). Since the eigenfunction in the inner region is proportional to $H_{n}(\xi)$, the integer $n$ determines the number of zeros of the eigenfunction. Figure 60.6 shows the normalized eigenfunction for the $n=0, n=1$, and $n=2$ modes for a smooth density profile. Observe the degradation of the matching between the outer WKB approximations and the inner solution as $n$ increases. This effect is due to the increasing magnitude of the higher-order corrective terms that scale as $\Sigma k_{1}(n) / k_{0}$. Thus we expect that the matching cannot be performed for $\Sigma k_{1}(n) / k_{0}>1$ and the number of branches does not exceed $N$ with $\Sigma k_{1}(N) / k_{0}>1$. Equation (19) has also been solved numerically, and the results have been compared with the analytical predictions. Figure 60.7 shows the unstable spectrum of an equilibrium configuration typical of direct-drive ICF with $g=5 \times 10^{15} \mathrm{~cm} / \mathrm{s}^{2}, V_{a}=7.5 \times 10^{4} \mathrm{~cm} / \mathrm{s}, A=0.9$, and $L_{0}=2 \mu \mathrm{m}$. Three branches have been found with $n=0, n=1$, and $n=2$. For this set of parameters $\Sigma k_{1}(3) / k_{0}=1.01$, and the predicted number of branches is indeed $N=3$. Equation (19) predicts the following values of the cutoff wave numbers: $k_{c}(n=0)=4.28 \mu \mathrm{m}^{-1}, k_{c}(n=1)=2.83 \mu \mathrm{m}^{-1}$, and $k_{c}(n=2)=$ $1.37 \mu \mathrm{m}^{-1}$. As expected the accuracy of Eq. (19) in predicting the cutoff wave number degrades as $n$ increases. For the same equilibrium parameters, the geometrical optics approximation
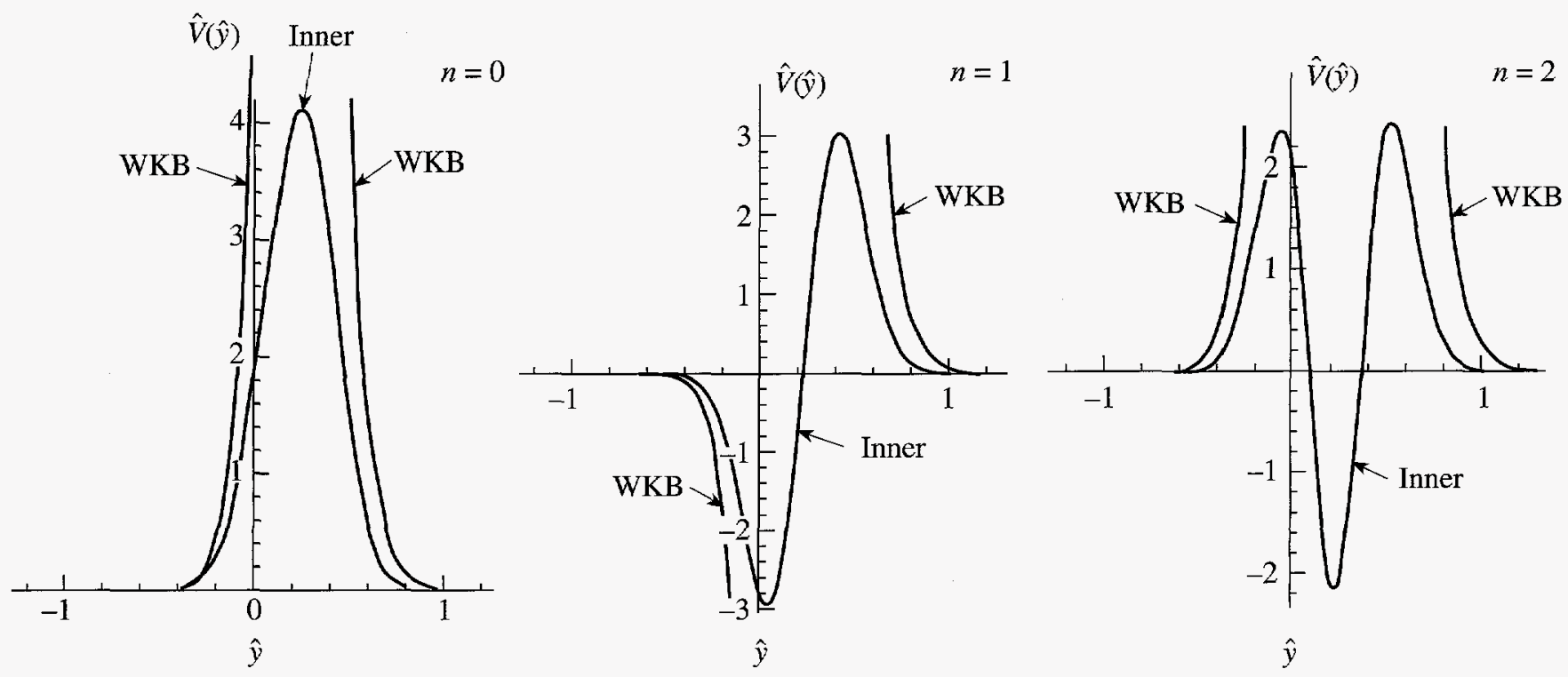

TC3515

Figure 60.6

Plot of the normalized eigenfunction $\hat{V}=\tilde{v}_{y}(\hat{y}) \exp \left[\left(\hat{y}-\hat{y}_{0}\right) / \sqrt{2} \in\right]$, for the $n=0,1,2$ modes and the following equilibrium parameters: $A=0.8, g=10^{15} \mathrm{~cm} / \mathrm{s}^{2}, v_{\mathbf{a}}=10^{4} \mathrm{~cm} / \mathrm{s}$, and $L_{0}=10 \mu \mathrm{m}$. 


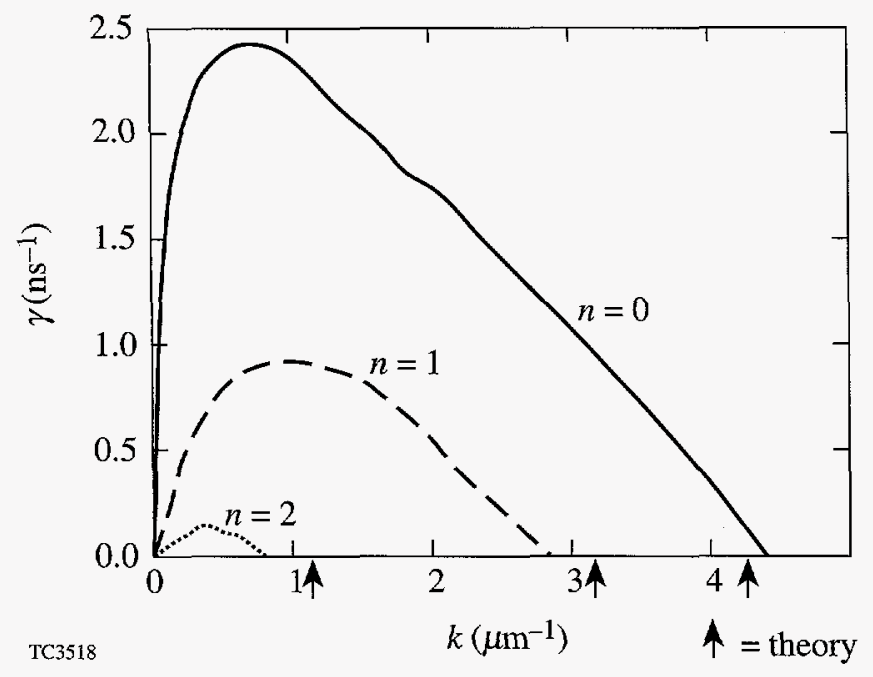

Figure 60.7

Plot of the growth rate versus the wave number for the $n=0,1,2$ branches and the following equilibrium parameters: $A=0.9, g=5 \times 10^{15} \mathrm{~cm} / \mathrm{s}^{2}, v_{\mathrm{a}}=$ $7.5 \times 10^{4} \mathrm{~cm} / \mathrm{s}$, and $L_{0}=2 \mu \mathrm{m}$.

of Ref. 8 predicts a cutoff of the $n=0$ mode at $k=5.5 \mu \mathrm{m}^{-1}$ with a relative error of approximately $30 \%$. To simplify Eq. (19), we focus on typical ICF equilibria with $A \approx 1$ and density profile given by Eq. (20) to determine an approximate value of $\hat{y}_{0}, \hat{y}_{0} \approx 0.25\left(\sqrt{8+\pi^{2}}-\pi\right)$. Substituting $\hat{y}_{0}$ into the expressions for $Q\left(\hat{y}_{0}\right)$ and $\hat{L}\left(\hat{y}_{0}\right)$, we derived the following approximate form of the cutoff wave number:

$$
k_{c}=k_{0}\left[1-2 \sqrt{\pi} \Sigma\left(n+\frac{7}{8}\right)\right] \quad(n=0,1,2 \ldots)
$$

and $k_{0} \simeq(\sqrt{2.17 / \pi}) / \Sigma L_{0}$. In addition to the cutoff wave number, the WKB approximation also provides the position where the short-wavelength modes are localized. The peak of the eigenfunction is located at the point $\hat{y}^{*}$, where $S^{\prime}\left(\hat{y}^{*}\right)=0$. Using Eq. (6) at $\hat{y}=\hat{y}^{*}$ we derive an equation for $\hat{y}^{*}$ :

$$
\Gamma^{2}\left(\hat{y}^{*}\right)=\sigma\left(\hat{y}^{*}\right)^{2} / \hat{L}\left(\hat{y}^{*}\right)
$$

For any given $\Gamma$, Eq. (22) can be solved for the unknown $\hat{y}^{*}$. It follows immediately that the mode corresponding to the cutoff wave number $(\Gamma=0)$ has the peak of the eigenfunction at $-\infty$, where $1 / \hat{L}=0$. More generally, it can be deduced from Eq. (22) that as the wave number of the mode increases and the growth rate decreases, the peak of the eigenfunction is shifted downstream in the light-fluid region. To verify the accuracy of the incompressible model in predicting the unstable spectrum for $\Sigma<1$, we compare the growth rates derived from Eqs. (2)(4) with the numerical results of Ref. 3, where the full set of fluid equations, including thermal transport, has been numerically solved. According to Ref. 3, the growth-rate dependence on the mode wave number is well fit by the following formula:

$$
\gamma=0.9 \sqrt{k g}-\beta k V_{a},
$$

where $\beta$ is an adjustable parameter varying between 3 and 4 . Figure 60.8 shows an unstable spectrum obtained from the numerical solution of the incompressible model for $\Sigma=0.14$ and Takabe's formula for $\beta=3-4$. Observe that the predictions of the incompressible model (for $\Sigma<1$ ) are in good agreement with the more general results of Ref. 3. For the same value of $\Sigma$, Eq. (19) yields the normalized cutoff wave number $\sqrt{k_{c} V_{a}^{2} / g}=0.26$. To check the validity of the incompressible mode for arbitrary equilibria, we also compare the incompressible spectrum for $\Sigma>>1$ with the result of Ref. 3. We find that the incompressible model predicts a larger ablative stabilization than Eq. (23). For $\Sigma \gg 1$, the incompressible growth rate can be written in the following form:

$$
\gamma=\sqrt{A K g}-k V_{a} \frac{1+A}{1-A}
$$

in agreement with the results of Ref. 4 . For $A \simeq 1$, Eq. (24) predicts a large stabilization that is not observed in the numerical simulations. ${ }^{3,5,9}$ We conclude that the incompressibility assumption breaks down for $\Sigma \gg 1$, and the effect of finite thermal conductivity must be retained. ${ }^{7}$

\section{Conclusions}

We have derived the physical optics approximation of the WKB theory applied to the incompressible ablative RayleighTaylor instability, and we have found the existence of multiple branches in the unstable spectrum. The calculated cutoff wave number is also reasonably accurate for configurations with rather steep density gradients or large ablation velocity $(\Sigma \leqslant 1)$. Although this is the first derivation of the multiple unstable branches in the presence of an equilibrium flow, this result is not surprising, as in the classical Rayleigh-Taylor instability, multiple modes also exist. However, no branch experiences a cutoff in the classical treatment, and the growth rate is monotically increasing with the mode wave number. 


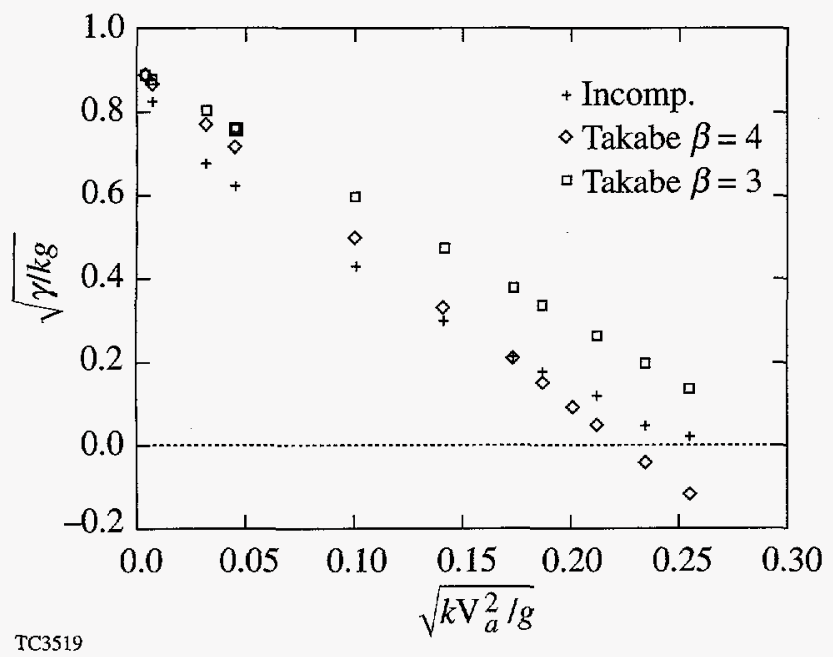

Figure 60.8

Plot of the normalized growth rate $\sqrt{\gamma / k g}$ versus the normalized wave number $\sqrt{k V_{a}^{2} / g}$, for the incompressible model (+), Eq. (23) with $\beta=3$ ( $\square$ ), and Eq. (23) with $\beta=4(\diamond)$. The equilibrium parameters are $L_{0}=0.5 \mu \mathrm{m}$, $g=10^{16} \mathrm{~cm} / \mathrm{s}^{2}, V_{a}=10^{5} \mathrm{~cm} / \mathrm{s}$, and $A=0.95$.

\section{ACKNOWLEDGMENT}

This work was supported by the U.S. Department of Energy Office of Inertial Confinement Fusion under Cooperative Agreement No. DE-FC0392SF19460, the University of Rochester, and the New York State Energy Research and Development Authority. The support of DOE does not constitute an endorsement by DOE of the views expressed in this article.

\section{REFERENCES}

1. Lord Rayleigh, Scientific Papers (Cambridge University Press, Cambridge, England, 1900), Vol. II, p. 200.

2. S. Bodner, Phys. Rev. Lett. 33, 761 (1974).

3. H. Takabe, K. Mima, L. Montierth, and R. L. Morse, Phys. Fluids 28, 3676 (1985).

4. H. J. Kull and S. I. Anisimov, Phys. Fluids 29, 2067 (1986).

5. J. H. Gardner, S. E. Bodner, and J. P. Dahlburg, Phys. Fluids B 3, 1070 (1991).

6. K. O. Mikaelian, Phys. Rev. A 46, 6621 (1992).

7. H. J. Kull, Phys. Fluids B 1, 170 (1989).

8. A. B. Bud'ko and M. A. Liberman, Phys. Rev. Lett. 68, 178 (1992).

9. C. P. Verdon, R. L. McCrory, R. L. Morse, G. R. Baker, D. I. Meiron, and S. A. Orszag, Phys. Fluids 25, 1653 (1982). 


\section{Ultrafast, All-Silicon Light Modulator}

The ability to send high-speed messages between integrated circuit devices requires both high-frequency receivers and transmitters. The vast majority of integrated circuits are made from silicon-based semiconductors. Suitable receivers of silicon integrated circuits can be constructed from either metal-semiconductor-metal photodiodes ${ }^{1}$ or $P$-type, intrinsic, $N$-type (PIN) photodiodes. These receivers are capable of acting as interconnection devices for frequencies up to tens of gigahertz. The transmitter half of this interconnection for silicon has proved to be more problematic. A semiconductor laser has yet to be made from silicon and may prove to be an impossible task. It may be possible to use an optical modulator in place of a semiconductor laser as the transmitter for siliconbased integrated circuits. The refractive index of silicon can be modified by the free-carrier density (the carrier-refraction effect) ${ }^{2}$ therefore, the refractive index can be changed by either the injection or depletion of free-carriers.

This effect has been utilized in all-silicon, light-intensity modulators. ${ }^{3-6}$ These devices have typically two components: one is an optical phase modulator; the other converts the phase modulation to intensity modulation. In the phase-modulation device, carrier injection into a lightly doped layer provides an extended interaction region wherein the index of refraction may be externally controlled. However, using carrier injection poses two undesirable concerns: the injected current density must be high, and the devices are inherently slow because their speed is limited by the carrier lifetime (nanoseconds to microseconds).

For practical applications, a high-speed, low-power-dissipation, high-modulation-depth, small-sized modulator is desirable. In this article we propose an ultrafast light modulator made on silicon-on-insulator (SOI) substrates. It is a field-effect device, accompanied naturally with low-power dissipation. This modulator is based on carrier depletion instead of carrier injection; hence, its speed may be limited only by the device capacitance and is estimated to be as high as $70 \mathrm{GHz}$. Most importantly, this modulator is not based on phase modulation. It is a Bragg reflector induced by electric field, which efficiently converts the small index modulation to light-intensity modulation.

Figure 60.9 shows the schematic view of the modulator. The SOI structure acts as an optical waveguide. On top of the silicon layer are interdigitated metal fingers, which form Schottky contacts with silicon. A microstrip line guides the electrical signal to the metal fingers. When the metal fingers are alternately biased, they form forward- and reverse-biased Schottky junctions with silicon. There are larger depleted regions on the reverse-biased sides, as shown in the shaded part of Fig. 60.10. In a $P$-type silicon layer with a hole concentration of $3 \times 10^{18} / \mathrm{cm}^{3}$, there is a refractive-index difference of $\Delta n=0.005$ between the depleted and undepleted regions, assuming an optical wavelength of $1.55 \mu \mathrm{m} .^{2}$ The alternately depleted and undepleted areas form a Bragg reflector in the optical waveguide. Applying a time-dependent voltage to the metal fingers modulates the shape of the depleted regions and, in turn, the reflectivity of the Bragg reflector and the light intensity.

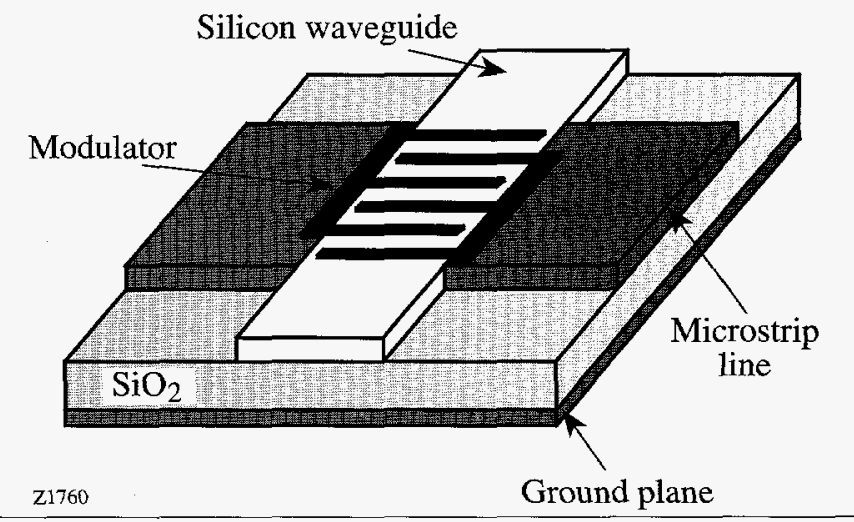

Figure 60.9

A schematic view of the waveguide modulator. The SOI structure acts as an optical waveguide. On top of the silicon layer are interdigitated metal fingers, which form Schottky contacts with silicon. A microstrip line guides the electrical signal to metal fingers. 

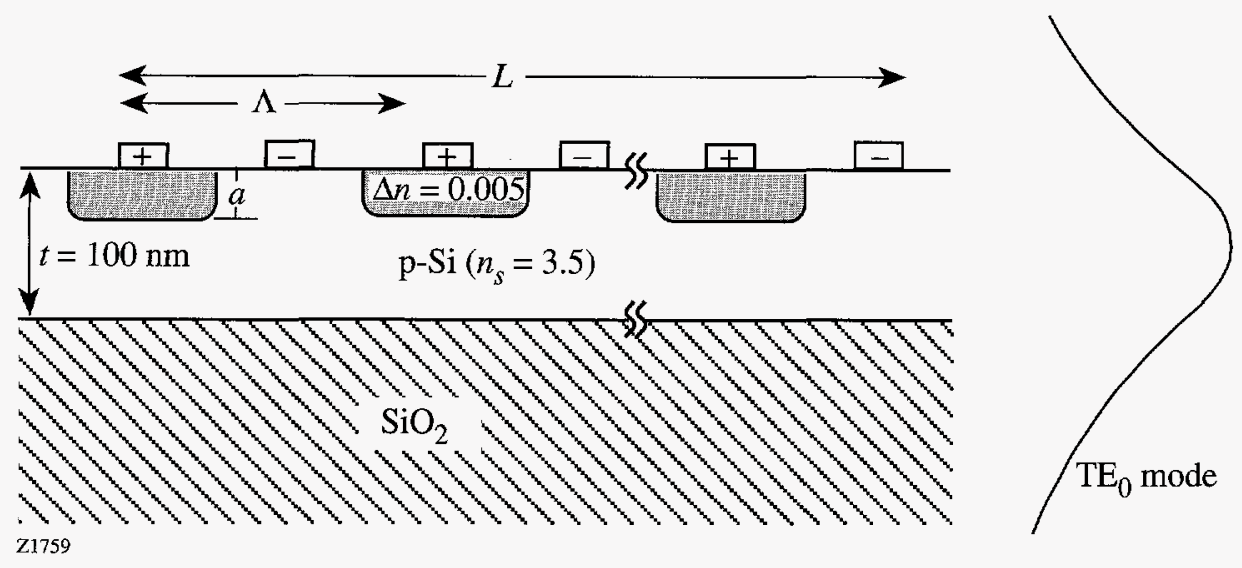

Figure 60.10

The reversed-biased Schottky contacts have larger depleted regions (shaded part), which have a larger refractive index. The alternating depleted and undepleted regions form a Bragg reflector in the waveguide. The electric-field-induced Bragg reflector converts the index modulation to light-intensity modulation. The $\mathrm{TE}_{0}$ mode profile is shown to the right of the modulator.

The reflectivity, or the modulation depth of this device, can be calculated by using coupled-mode theory. ${ }^{7}$ The modulation depth is maximized when the Bragg condition is satisfied, i.e., $\Lambda=\lambda / 2$, where $\Lambda$ is the period of finger electrodes as shown in Fig. 60.10 and $\lambda$ is the light wavelength in the guiding layer. In this case, the reflectivity can be expressed as $R=\tanh ^{2}(\kappa L)$, where $L$ is the interaction length of the modulator and $\kappa$ is the coupling constant. If the depleted region is assumed to have a rectangular shape and equal length with the undepleted region (as shown in Fig. 60.10), then $\kappa$ is given by ${ }^{7}$

$$
\kappa=\frac{\omega \varepsilon_{0}\left[\left(n_{s}+\Delta n\right)^{2}-n_{s}^{2}\right]}{4 \pi} \times \int_{-a}^{0}|E(x)|^{2} d x,
$$

where $\omega$ is the frequency of the optical wave, $E(x)$ is the normalized wave solution of the optical waveguide, $a$ is the depletion width, and $n_{s}$ is the refractive index of the silicon layer. Here, $n_{s}$ is taken to be 3.50 . The indices of air and silicon dioxide are chosen to be 1 and 1.45. The thickness of the guiding layer is chosen to be $100 \mathrm{~nm}$ for single-mode operation and to sufficiently guide the $\mathrm{TE}_{0}$ mode of light with wavelength of $1.55 \mu \mathrm{m}$. (For TM mode, the coupling constant is much smaller, characteristic of Bragg reflectors.) The guided wavelength in this SOI waveguide is $0.72 \mu \mathrm{m}$; thus, to satisfy the Bragg condition the period of the finger electrodes is $360 \mathrm{~nm}$.

Since $a$ is a function of the electrode voltage, the modulation depth is also voltage dependent. The modulation depth as a function of biasing voltage is shown in Fig. 60.11 for several interaction lengths. In this calculation, the hole concentration of the silicon layer is set to be $3 \times 10^{18} / \mathrm{cm}^{3}$, and the built-in voltage of the Schottky contact is $0.5 \mathrm{~V}$, which is typical for metal-semiconductor contacts. It shows that, with $5-\mathrm{V}$ bias, a modulator with interaction length of 300 and

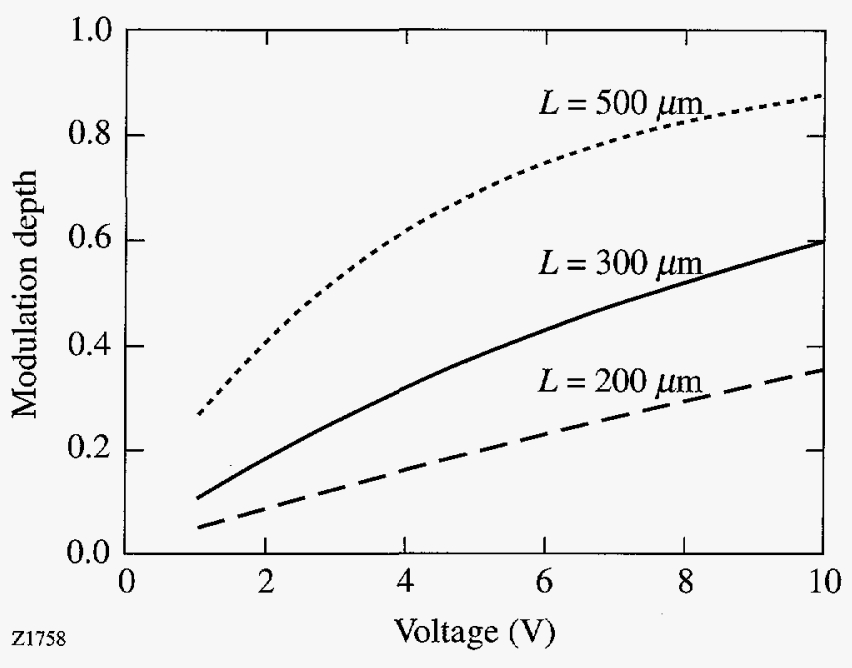

Figure 60.11

The modulation depth of modulators for $\mathrm{TE}_{0}$ mode with different interaction lengths is shown as a function of biasing voltage. The thickness of the guiding layer is $100 \mathrm{~nm}$, and the doping is $P=3 \times 10^{18} / \mathrm{cm}^{3}$.

$500 \mu \mathrm{m}$ has a modulation depth of about $40 \%$ and $70 \%$, adequate for most applications.

In an actual device, the depletion region is, of course, not rectangular. Such a profile has been simulated using the MEDICI program ${ }^{8}$ and is shown in Fig. 60.12. However, since the modulation depth depends only on the fundamental Fourier component of the shape of the depletion region, we expect the simulated results in Fig. 60.11 to closely approximate that of an actual device.

A similar structure has been used by Alferness and $\mathrm{Buhl}^{9}$ on an electro-optic modulator, which provides the conversion between TE and TM modes. However, the co-directional coupling between $\mathrm{TE}$ and $\mathrm{TM}$ modes requires a much larger electrode period to satisfy the Bragg condition. As a result, 


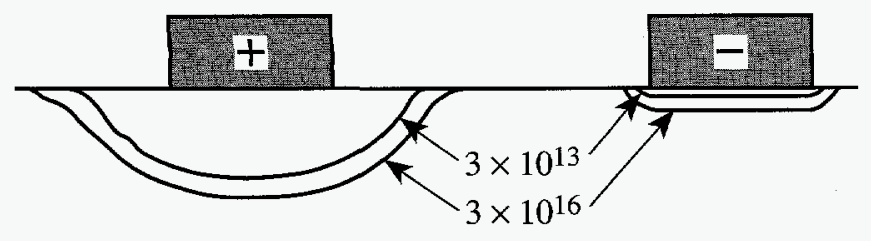

Si, $P=3 \times 10^{18} / \mathrm{cm}^{3}$

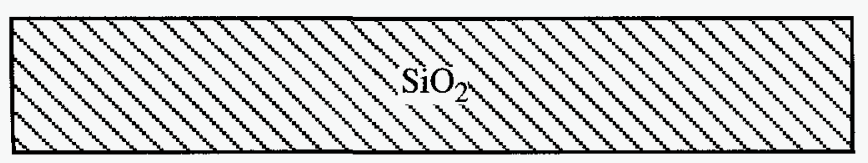

Z1757

Figure 60.12

The shape of the depleted region is examined by using the MEDICI simulation program. There are alternately depleted and undepleted regions under the reverse- and forward-biased finger electrodes. The finger width and spacing are 60 and $120 \mathrm{~nm}$, respectively. The doping of silicon is $P=3 \times 10^{18} / \mathrm{cm}^{3}$; the bias voltage is $5 \mathrm{~V}$.

the size of this TE $\leftrightarrow$ TM modulator is large (of the order of millimeters). Furthermore, Alferness's modulator is not integrable with silicon planar circuits because an external polarizer is required to convert polarization modulation to intensity modulation.

The fabrication of our proposed modulator is feasible with existing fabrication techniques. Bond-and-etchback SOI wafers have proven to be a good optical waveguide material with scattering loss of about $3.3 \mathrm{~dB} / \mathrm{cm}$ for $\lambda=1.55 \mu \mathrm{m} .{ }^{10}$ A grating coupler can be used to couple light into or out of the optical waveguide. The nanometer-sized finger electrodes can be fabricated by using modern electron-beam lithography. ${ }^{11} \mathrm{~A}$ thin silicon-dioxide layer can be placed between the finger electrodes and the silicon layer to increase the breakdown voltage and avoid direct metal loading, which contributes to the attenuation of the guided wave.

The operational speed of this device is predominantly limited by the intrinsic device RC time constant, where $\mathrm{R}$ is the line impedance connecting to the modulator and $\mathrm{C}$ is the modulator capacitance. Parasitic capacitance for this structure is negligible, as shown from our previous measurements on a photodetector with similar structure. ${ }^{11}$ For the inherent RC time constant, there is a trade-off between the modulation depth and the interaction length of the modulator, which affects its bandwidth. A modulator with $60-\mathrm{nm}$ finger width, 120 -nm finger spacing, $3-\mu \mathrm{m}$ finger length, and $300-\mu \mathrm{m}$ interaction length is calculated to have a capacitance of $0.22 \mathrm{pf}^{12}$ (For the interdigitated structure, the capacitance is propor- tional to the interaction length and inversely proportional to the finger period.) If a microstrip line with $10-\Omega$ impedance is used, the bandwidth of this modulator is $1 / 2 \pi \mathrm{RC}$, or about $70 \mathrm{GHz}$. A thinner guiding layer may improve the bandwidth in two ways: by increasing the coupling constant, thus allowing a shorter interaction length, and by increasing the guided wavelength; as a result, the period of the metal fingers is increased and the capacitance is decreased.

For practical applications, the insertion loss to this modulator should be considered but is dependent on the configuration chosen. Free-carrier absorption would also contribute to the loss. For the proposed sample device, this effect is estimated to be about $0.6 \mathrm{~dB}^{2}$. Finally, for this Bragg reflector, an optical isolator such as a directional coupler at the insertion point is clearly necessary to prevent multiple reflections.

In conclusion, an ultrafast, all-silicon, light-intensity modulator has been proposed. An electric-field-induced Bragg reflector efficiently converts the small refractive-index modulation to light-intensity modulation. A modulator with $300-\mu \mathrm{m}$ interaction length is shown to have a modulation depth about $40 \%$ with 5-V bias. Because it is a field-effect device, it has power dissipation dominated by leakage current and the resistance of the metal fingers. The speed of the modulator is limited only by the RC time constant, and a sample device has a bandwidth about $70 \mathrm{GHz}$. The same interdigitated structure has been demonstrated by us ${ }^{13}$ to be usable as a picosecond photodetector. Both device structures are compatible with silicon circuits, lending themselves to potential use in integrated electronic-optoelectronic systems.

\section{ACKNOWLEDGMENT}

This work was supported by the University Research Initiative at the University of Rochester sponsored by the Army Research Office grant No. DAAL03-92-G-0112. We would like to acknowledge Dr. Xing Zhou for his computation of Fig. 60.12 .

\section{REFERENCES}

1. S. Alexandrou, C.-C. Wang, T. Y. Hsiang, M. Y. Liu, and S. Y. Chou, Appl. Phys. Lett. 62, 2507 (1993).

2. R. A. Soref and B. R. Bennett, IEEE J. Quantum Electron. QE-23, 123 (1987).

3. B. R. Hemenway, O. Solgaard, and D. M. Bloom, Appl. Phys. Lett. 55, 349 (1989).

4. B. R. Hemenway, O. Solgaard, A. A. Godil, and D. M. Bloom, IEEE Photonics Technol. Lett. 2, 262 (1990).

5. O. Solgaard, A. A. Godil, B: R. Hemenway, and D. M. Bloom, IEEE Photonics Technol. Lett. 2, 640 (1990). 
6. X. Xiao, J. C. Sturm, K. K. Goel, and P. V. Schwartz, IEEE Photonics Technol. Lett. 3, 230 (1991).

7. A. Yariv and P. Yeh, Optical Waves in Crystals: Propagation and Control of Laser Radiation (Wiley, New York, 1984), Chap. 11.

8. Computer code MEDICI (Technology Modeling Associates, Inc., 3950 Fabian Way, Palo Alto, CA 94303-4605, USA, 1993).

9. R. C. Alferness and L. L. Buhl, Opt. Lett. 5, 473 (1980).

10. A. F. Evans, D. G. Hall, and W. P. Maszara, Appl. Phys. Lett. 59, 1667 (1991).
11. M. Y. Liu, S. Y. Chou, T. Y. Hsiang, S. Alexandrou, and R. Sobolewski, J. Vac. Sci. Technol. B 10, 2932 (1992).

12. Y. C. Lim and R. A. Moore, IEEE Trans. Electron Devices 15, 173 (1968).

13. C.-C. Wang, S. Alexandrou, D. Jacobs-Perkins, and T. Y. Hsiang, "Comparison of the Picosecond Characteristics of Silicon and Siliconon-Sapphire Metal-Semiconductor-Metal Photodiodes," Appl. Phys. Lett. 64, 3578 (1994). 


\section{Angular Dependence of Stimulated Brillouin Scattering in Homogeneous Plasma}

Stimulated Brillouin scattering (SBS) ${ }^{1}$ is the decay of an incident, or pump, light wave (0) into a frequency-downshifted, or Stokes, light wave (1) and an ion-acoustic wave (2). The conservation of energy and momentum in this process is reflected in the frequency and wave-vector matching conditions

$$
\omega_{0}=\omega_{1}+\omega_{2}, \mathbf{k}_{0}=\mathbf{k}_{1}+\mathbf{k}_{2}
$$

the second of which is illustrated in Fig. 60.13(a). For future reference, notice that the ion-acoustic wave number $k_{2}$ is maximal for directly backward scatterering and is equal to zero for directly forward scattering.

(a)

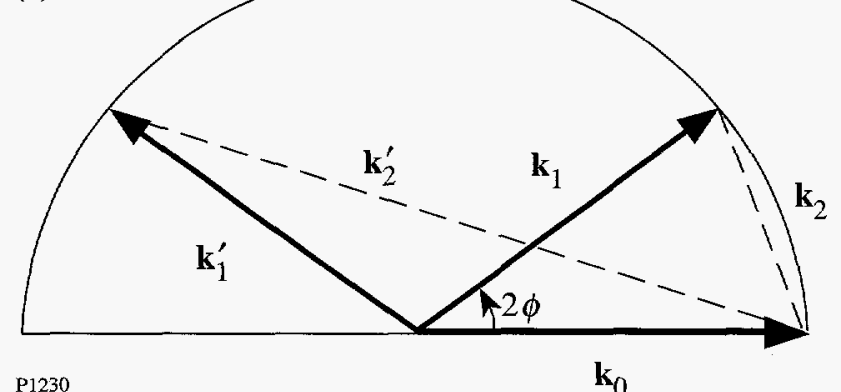

$\mathrm{P} 1230$

$\mathbf{k}_{0}$

(b)

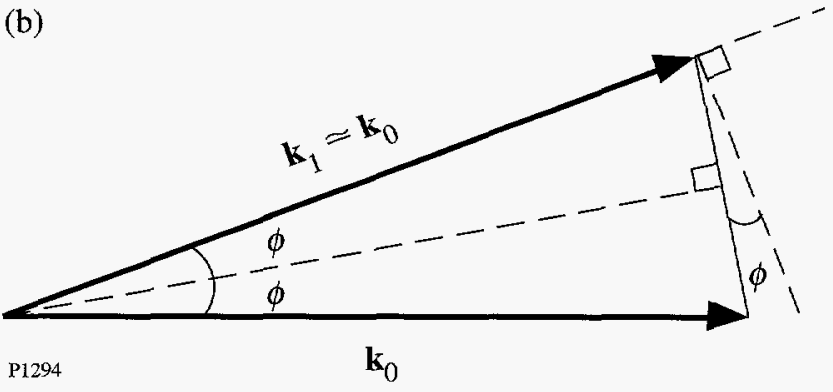

Figure 60.13

Interaction geometry for SBS. The angular dependence of $\gamma_{0}$, the temporal growth rate of SBS, stems from the fact that $k_{2} \approx 2 k_{0} \sin \phi$.
SBS is important in the field of inertial confinement fusion $(\mathrm{ICF})^{2}$ because it can scatter the incident light away from the target, thereby reducing the amount of energy available to drive the compressive heating of the nuclear fuel. ICF experiments have involved plasmas with density and temperature scale lengths shorter than $100 \mu \mathrm{m}$ for much of the last two decades, and analyses of parametric instabilities such as SBS have focused on the effects of these plasma inhomogeneities. ${ }^{3}$ However, current experiments are designed to produce plasma conditions relevant to the proposed National Ignition Facility and involve plasmas with millimeter scale lengths. There has been a resurgence of interest in the predictions of the simpler homogeneous-plasma model because such long scale lengths diminish the importance of the effects of plasma inhomogeneities.

Traditionally, analyses of SBS begin with the determination of $\gamma_{0}$, the temporal growth rate of SBS in an infinite homogeneous plasma. It is well known that $\gamma_{0}$ is maximal for directly backward scattering and is equal to zero for directly forward scattering. ${ }^{1}$ Physically, this behavior occurs because the ponderomotive force that drives the ion-acoustic wave is proportional to the square of the ion-acoustic wave number. Recognition of this behavior has led to the commonly held belief that backward SBS should dominate experiments involving long-scale-length plasmas.

The problem with this conclusion is that SBS cannot grow exponentially in time, with growth rate $\gamma_{0}$, for a time longer than that taken for light to cross the interaction region. ${ }^{4}$ In current experiments, this transit time does not exceed a few picoseconds. In contrast, the temporal pulsewidth of a typical laser is a few nanoseconds, and the initial period of temporal growth is followed by a much longer period of spatiotemporal growth. If the incident laser intensity is less than the threshold intensity for the absolute instability, SBS will eventually saturate due to the convection of the Stokes and ion-acoustic waves out of the interaction region. If this convective saturation occurs on a nanosecond time scale, the angular dependence of the scattered light will be determined predominantly 
by the physics of steady-state amplification. The goal of the work presented in this article is to determine how the convective amplification of the Stokes wave depends on the scattering angle and the conditions under which a steady-state analysis is applicable.

\section{Governing Equations}

The starting point for this investigation is Maxwell's wave equation

$$
\left(\partial_{t t}^{2}+\omega_{e}^{2}-c^{2} \nabla^{2}\right) \mathbf{A}_{h}=-\omega_{e}^{2} n_{l} \mathbf{A}_{h}
$$

for the vector potential, together with the ion-acoustic wave equation

$$
\left(\partial_{t t}^{2}-c_{s}^{2} \nabla^{2}\right) n_{l}=\frac{1}{2} c_{s}^{2} \nabla^{2}\left\langle\mathbf{A}_{h} \cdot \mathbf{A}_{h}\right\rangle
$$

These equations were derived ${ }^{5,6}$ under the assumption that the backgound plasma is uniform and at rest. The vector potential is normalized in such a way that $\mathbf{A}_{h}$ represents the velocity of electrons oscillating in the high-frequency electric field divided by a characteristic speed that is approximately equal to the electron thermal speed and $n_{l}$ represents the low-frequency electron-density fluctuation associated with the ion-acoustic wave divided by the background elecron density. The symbol $\omega_{e}$ denotes the electron plasma frequency, ${ }^{6}$ and the symbol $c_{s}$ denotes the ion-acoustic speed. ${ }^{7}$ Only the low-frequency plasma response to the ponderomotive force was retained, as is signified by the \langle\rangle in Eq. (3).

SBS involves the interaction of two light waves and an ionacoustic wave. These waves are coupled because of the electron current and the ponderomotive force, both of which are nonlinear. Because the effects of this coupling manifest themselves as slow spatial and temporal modulations of the wave amplitudes, the vector potential is written as

$$
\begin{aligned}
\mathbf{A}_{h}(\mathbf{r}, t) & =\mathbf{z}\left\{A_{0} \exp \left[i\left(\mathbf{k}_{0} \cdot \mathbf{r}-\omega_{0} t\right)\right]\right. \\
& \left.+A_{1}(x, y, t) \exp \left[i\left(\mathbf{k}_{1} \cdot \mathbf{r}-\omega_{1} t\right)\right]\right\},
\end{aligned}
$$

where $\mathbf{k}_{0}$ and $\mathbf{k}_{1}$ are parallel to the $x y$ plane, $\left(\omega_{0}, \mathbf{k}_{0}\right)$ and $\left(\omega_{1}, \mathbf{k}_{1}\right)$ satisfy the dispersion relation $\omega^{2}=\omega_{e}^{2}+c^{2} k^{2}$, and

$$
\nabla A_{1} \ll \mathbf{k}_{1} A_{1}, \partial_{t} A_{1} \ll \omega_{1} A_{1}
$$

In this linearized analysis of the initial evolution of SBS, the amplitude of the incident wave is held fixed. The electrondensity fluctuation is written as

$$
n_{l}(\mathbf{r}, t)=A_{2}(x, y, t) \exp \left[i\left(\mathbf{k}_{2} \cdot \mathbf{r}-\omega_{2} t\right)\right]
$$

where $\omega_{2}=c_{s} k_{2}$ and $A_{2}$ satisfies an equation similar to Eq. (5). By substituting Ansaetze (4) and (6) into Eqs. (2) and (3), and collecting terms of like frequency and wave vector, one finds that

$$
\begin{aligned}
& \left(\partial_{t}+\mathbf{v}_{1} \cdot \nabla+v_{1}\right) B_{1}=\gamma_{0} B_{2}, \\
& \left(\partial_{t}+\mathbf{v}_{2} \cdot \nabla+v_{2}\right) B_{2}=\gamma_{0} B_{1}
\end{aligned}
$$

In Eqs. (7) the dependent variables

$$
B_{1}=\omega_{1}^{1 / 2} A_{1}, \quad B_{2}=-i \omega_{e} A_{2}^{*} / \omega_{2}^{1 / 2}
$$

are proportional to the action amplitudes of the Stokes and ionacoustic waves, respectively, and the group velocities

$$
\mathbf{v}_{1}=c^{2} \mathbf{k}_{1} / \omega_{1}, \quad \mathbf{v}_{2}=c_{s} \mathbf{k}_{2} / k_{2}
$$

Phenomenological damping terms were added to each wave equation. The Stokes wave is damped by electron-ion collisions $^{6}$ and

$$
v_{1}=\omega_{e}^{2} v_{e i} / 2 \omega_{1}^{2}
$$

For plasmas in which $\left(T_{i} / m_{i}\right)^{1 / 2} \ll \omega_{2} / k_{2} \ll\left(T_{e} / m_{e}\right)^{1 / 2}$, the Landau contribution to the ion-acoustic damping rate ${ }^{7}$ is given by

$$
\frac{v_{2}}{c_{s} k_{2}} \approx\left(\frac{\pi}{8}\right)^{1 / 2}\left[\left(\frac{Z T_{e}}{T_{i}}\right)^{3 / 2} \exp \left(-\frac{Z T_{e}}{2 T_{i}}-\frac{3}{2}\right)+\left(\frac{Z m_{e}}{m_{i}}\right)^{1 / 2}\right]
$$

Relation (11) is based on the assumption that the ion-acoustic wavelength is much longer than the electron Debye length, an assumption that is also inherent in Eq. (3). The coupling parameter 


$$
\gamma_{0}=\frac{\omega_{e} c_{s} k_{2} A_{0}}{2\left(\omega_{1} c_{s} k_{2}\right)^{1 / 2}}
$$

where the peak amplitude of the incident wave is given by the convenient formula

$$
2 A_{0} \approx \frac{1.9 \times 10^{-8}\left[I\left(\mathrm{~W} / \mathrm{cm}^{2}\right)\right]^{1 / 2} \lambda_{0}(\mu \mathrm{m})}{\left[T_{e}(\mathrm{keV})\right]^{1 / 2}\left[1+3\left(T_{i} / Z T_{e}\right)\right]^{1 / 2}}
$$

It is clear from Fig. 60.13(b) that $k_{2} \approx 2 k_{0} \sin \phi$. Thus, one can exhibit the angular dependence of the coupling parameter and the ion-acoustic damping rate by writing

$$
\gamma_{0}(\phi)=\gamma_{b}(\sin \phi)^{1 / 2}, \quad v_{2}(\phi)=v_{b} \sin \phi
$$

where the subscript $b$ denotes values appropriate for backward SBS. In a similar vein,

$$
v_{2 x}=-c_{s} \sin \phi
$$

\section{Steady-State Amplification of the Stokes Wave}

In experiments typical of ICF the growth of the Stokes wave is initiated within the plasma by ion-acoustic fluctuations. This process is referred to in the nonlinear optics literature as Stokes generation and has been analyzed by Boyd, Rzazewski, and Narum. ${ }^{8}$ By neglecting the convection of the acoustic wave, they showed that the time-asymptotic Stokes output can be written as the product of two terms: a source factor that depends on the amplitude of the acoustic fluctuations and an exponential gain factor that does not depend on the amplitude of the acoustic fluctuations. Furthermore, the exponent of the gain factor is identical to that for the Stokes amplification process, in which an externally generated Stokes wave is amplified convectively as it propagates through the medium.
A preliminary analysis of Stokes generation in a plasma, which includes the convection of the ion-acoustic wave, has been made by McKinstrie et al. ${ }^{9}$ The inclusion of ion-acoustic wave convection allows SBS to be absolutely unstable when the laser intensity is sufficiently high. However, in the convectively unstable regime, the Stokes output tends to a time-asymptotic steady state, and the gain exponents for Stokes generation and Stokes amplification are identical, as one should expect. Thus, one can avoid the mathematical difficulties associated with the analysis of Stokes generation and still obtain useful information regarding the angular dependence of SBS by analyzing Stokes amplification in the convectively unstable regime.

The interaction geometry for Stokes amplification is shown in Fig. 60.14(a). Initially,

$$
B_{1}(x, y, 0)=0, B_{2}(x, y, 0)=0 .
$$

At time $t=0$ the leading edge of the externally generated Stokes wave enters the plasma with unit amplitude and initiates the instability. The ion-acoustic wave has zero amplitude at its entrance boundaries. For simplicity, we assume that the plasma is square and that its boundaries are aligned with the $x$ and $y$ axes. Thus, for $t>0$,

$$
B_{1}(0, y, t)=1, B_{2}(x, 0, t)=0, B_{2}(l, y, t)=0 .
$$

For a plasma with sides of length $100 \mu \mathrm{m}$, the transit time of the Stokes wave is $0.3 \mathrm{ps}$. In contrast, the transit time of the ion-acoustic wave is of the order of $7\left(m_{i} / m_{e}\right)^{1 / 2}$ ps for an electron temperature of $1 \mathrm{keV}$. Since the duration of a typical laser pulse is of the order of $1 \mathrm{~ns}$, the information that a side boundary is present at $y=0$ will not reach the plasma interior until the later stages of a typical experiment. Since the amplitude of the Stokes wave at its entrance boundary is independent of $y$, the initial evolution of SBS in the plasma interior is approximately one-dimensional and is governed approximately (a)

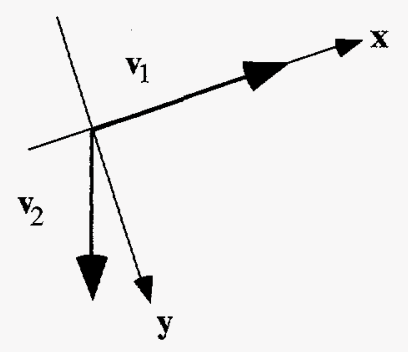

$\mathrm{P} 1234$ (b)

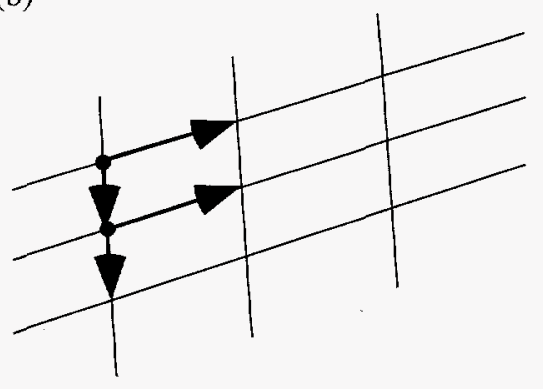

Figure 60.14

Group-velocity geometry for SBS. (a) The $x$ component of $\mathbf{v}_{2}$ is always negative. (b) The initial evolution of SBS is approximately one-dimensional because the convection of portions of the ion-acoustic wave away from a particular Stokes ray is compensated by the convection of neighboring portions of the ion-acoustic wave toward that Stokes ray. 
by the equations

$$
\begin{aligned}
& \left(\partial_{t}+v_{1 x} \partial_{x}+v_{1}\right) B_{1}=\gamma_{0} B_{2}, \\
& \left(\partial_{t}-v_{2 x} \partial_{x}+v_{2}\right) B_{2}=\gamma_{0} B_{1},
\end{aligned}
$$

where $v_{2 x}$ is defined to be positive.

Physically, this simplification occurs because the convection of portions of the ion-acoustic wave away from a particular Stokes ray is compensated by the convection of neighboring portions of the ion-acoustic wave toward that Stokes ray, as shown in Fig. 60.14(b). Notice that the preceding argument did not depend sensitively on the shape or alignment of the plasma.

The consequences of the one-dimensional model [Eqs. (18)] will now be studied, under the assumption that the instability grows and saturates convectively in a time that is short compared to the duration of the laser pulse. In steady state, the boundary conditions are

$$
B_{1}(0)=1, \quad B_{2}(l)=0
$$

It is not difficult to show that

$$
\begin{aligned}
& B_{1}(x)=\frac{\left\{\beta \cosh [\beta(l-x)]+\alpha_{s} \sinh [\beta(l-x)]\right\} \exp \left(\alpha_{d} x\right)}{\left[\beta \cosh (\beta l)+\alpha_{s} \sinh (\beta l)\right]}, \\
& B_{2}(x)=\frac{\gamma_{0} \sinh [\beta(l-x)] \exp \left(\alpha_{d} x\right)}{v_{2 x}\left[\beta \cosh (\beta l)+\alpha_{s} \sinh (\beta l)\right]},
\end{aligned}
$$

where the auxiliary parameters

$$
\begin{aligned}
& \alpha_{1}=v_{1} / v_{1 x}, \alpha_{2}=v_{2} / v_{2 x}, \\
& \alpha_{s}=\left(\alpha_{2}+\alpha_{1}\right) / 2, \alpha_{d}=\left(\alpha_{2}-\alpha_{1}\right) / 2,
\end{aligned}
$$

and

$$
\beta=\left(\alpha_{s}^{2}-\gamma^{2}\right)^{1 / 2}, \quad \gamma=\gamma_{0} /\left(v_{1 x} v_{2 x}\right)^{1 / 2}
$$

The wave amplitudes $B_{1}(x)$ and $\left(v_{2 x} / v_{1 x}\right)^{1 / 2} B_{2}(x)$ are plotted in Fig. 60.15 for the case in which $\alpha_{1} l=0, \alpha_{2} l=52$, and $\gamma l=17$. These parameters, which are used throughout most of this article, correspond to a singly ionized plasma with a background electron density of $10^{19} / \mathrm{cm}^{3}$, an electron temperature of $1 \mathrm{keV}$, an ion temperature of $0.1 \mathrm{keV}$ and a length of $100 \mu \mathrm{m}$, and a laser intensity of $5 \times 10^{14} \mathrm{~W} / \mathrm{cm}^{2}$.

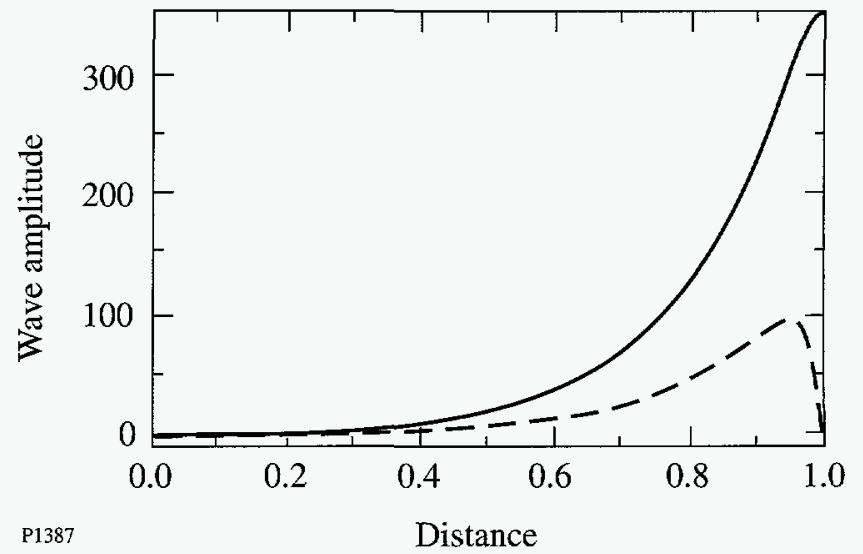

Figure 60.15

Steady-state amplitudes of the Stokes (solid line) and ion-acoustic (broken line) waves [Eqs. (20)] plotted as functions of distance for the case in which the damping parameters $\alpha_{1} l=0$ and $\alpha_{2} l=52$, and the pump-strength parameter $\gamma l=17$. These parameters are all independent of the scattering angle $2 \phi$. Distance is measured in units of the plasma length $l$.

It follows from the first of Eqs. (20) that the convective gain associated with the Stokes amplification process, which is defined as $B_{1}(l) / B_{1}(0)$, is given by ${ }^{10}$

$$
G=\frac{\beta \exp \left(\alpha_{d} l\right)}{\beta \cosh (\beta l)+\alpha_{s} \sinh (\beta l)} .
$$

It follows from Eqs. (14), (21), and (22) that the auxiliary parameters used in Eq. (23) are all independent of the scattering angle $2 \phi$. This fact has two important consequences.

First, although $\gamma_{0}$, the temporal growth rate of SBS, is proportional to $(\sin \phi)^{1 / 2}$, the steady-state convective gain associated with this one-dimensional model of Stokes amplification is independent of the scattering angle. ${ }^{11}$ Typically, $\alpha_{1} \ll \alpha_{2}$. When $\beta l \ll 1$ one can write

$$
G \propto \exp (g l)
$$

where the spatial growth rate

$$
g \approx \frac{v_{b}}{2 v_{2}}-\left[\left(\frac{v_{b}}{2 v_{2}}\right)^{2}-\frac{\gamma_{b}^{2}}{v_{1} v_{2}}\right]^{1 / 2} .
$$


In typical nonlinear optics experiments the low acoustic speed results in strong spatial damping of the acoustic wave and

$$
g \approx \gamma_{b}^{2} / v_{1} v_{b}
$$

Equation (26) is independent of the acoustic speed, as one should expect. The exact and approximate gain exponents [Eqs. (25) and (26)] are plotted as functions of the pumpintensity parameter $(\gamma l)^{2}$ in Fig. 60.16 for the case in which $\alpha_{2} l=52$. For a pump-intensity parameter of 600 , which corresponds to a laser intensity of approximately $10^{15} \mathrm{~W} / \mathrm{cm}^{2}$, use of the approximate gain exponent leads to an estimate of the intensity of the Stokes output that is five orders of magnitude too low! This result shows the importance of retaining the effects of ion-acoustic wave convection in analyses of SBS in plasmas. ${ }^{9}$

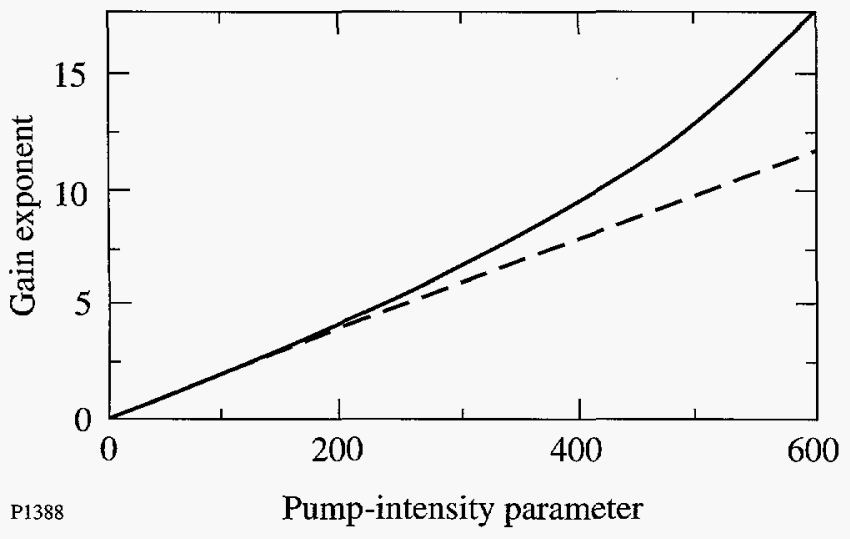

Figure 60.16

Exact (solid line) and approximate (broken line) gain exponents of SBS [Eqs. (25) and (26)] plotted as functions of the pump-intensity parameter $(\gamma l)^{2}$ for the case in which $\alpha_{1} l=0$ and $\alpha_{2} l=52$. The large discrepancy between the gain exponents demonstrates the importance of ion-acoustic wave convection. This result distinguishes plasmas from most other nonlinear optical media.

It is well known that the steady-state convective gain becomes infinite when the denominator of Eq. (23) vanishes. This singularity can occur when $\gamma>\alpha_{2} / 2$ and signifies the onset of absolute instability. The second important consequence of the fact described after Eq. (23) is that, in the context of this one-dimensional model, the absolute instability condition for SBS is independent of the scattering angle. ${ }^{11}$ Physically, the existence of absolute instability stems from the fact that the component of the ion-acoustic velocity in the direction of the Stokes velocity is always negative [Eq. (15)].

The results described in the preceding two paragraphs are in apparent contradiction to the well known fact that $\gamma_{0} \rightarrow 0$ as $\phi \rightarrow 0$ and, hence, that directly forward SBS does not exist. One can resolve this apparent contradition by realizing that the response of the acoustic wave to the ponderomotive force of the pump and Stokes waves is like that of a driven harmonic oscillator, in which the oscillator takes several damping times to attain its steady-state response. Since $v_{2}$ is proportional to $\sin \phi$, the saturation time associated with this one-dimensional model of SBS tends to infinity as the scattering angle tends to zero, and use of the steady-state gain formula is inappropriate in this limit.

\section{Transient Evolution of SBS}

The exact solutions of Eqs. (18) have been obtained for a finite plasma by Bobroff and Haus ${ }^{12}$ and by Williams and McGowan. ${ }^{13}$ However, these solutions are written in terms of infinite sums of modified Bessel functions, and, because of their complexity, a different approach is taken here. Since the duration of a typical laser pulse is comparable to the transit time of the acoustic wave, one might suspect that the initial evolution of SBS does not depend sensitively on the finiteplasma boundary conditions. In this vein, consider the evolution of SBS in an infinite plasma. The arrival of an externally generated Stokes wave at $x=0$ is modeled by adding a source term

$$
S_{1}(x, t)=v_{1 x} \delta(x) H(t)
$$

to the first of Eqs. (18). The corresponding temporal growth and saturation of the Stokes output $B_{1}(l, t)$ will be studied analytically. The results obtained from this approximate analysis will then be verified by solving Eqs. (18) numerically for a finite plasma.

It is shown in the Appendix that, consistent with Eq. (27), the Stokes wave evolves according to

$$
B_{1}(x, t)=v_{1 x} \int_{0}^{t} G_{11}\left(x, t^{\prime}\right) d t^{\prime},
$$

where 


$$
\begin{aligned}
G_{11}(x, t) & =\frac{\gamma_{0}}{v_{1 x}+v_{2 x}}\left(\frac{x+v_{2 x} t}{v_{1 x} t-x}\right)^{1 / 2} \\
& \times \quad I_{1}\left(\frac{2 \gamma_{0}\left[\left(x+v_{2 x} t\right)\left(v_{1 x} t-x\right)\right]^{1 / 2}}{v_{1 x}+v_{2 x}}\right) \\
& \times \exp \left[-\frac{v_{1}\left(x+v_{2 x} t\right)}{v_{1 x}+v_{2 x}}-\frac{v_{2}\left(v_{1 x} t-x\right)}{v_{1 x}+v_{2 x}}\right] \\
& \times H\left(x+v_{2 x} t\right) H\left(v_{1 x} t-x\right) \\
& +H\left(x+v_{2 x} t\right) \delta\left(v_{1 x} t-x\right) \exp \left(-v_{1} t\right)
\end{aligned}
$$

is the Green function that describes the effect on the Stokes wave at the point $(x, t)$ of an impulse applied to it at the point $(0,0)$. The impulse response $G_{11}(l, t)$ is displayed in Fig. 60.17 for the case in which $\alpha_{1} l=0, \alpha_{2} l=52$, and $\gamma l=17$. The impulse response grows in time until it attains its maximal amplitude at $t=t_{*}$. Subsequently, it decays in a time comparable to the growth time. Since the time-asymptotic Stokes output is proportional to the area under the impulse response curve [Eq. (28)], it is reasonable to define the saturation time $t_{s}$ as $2 t_{*}$. Using the fact that

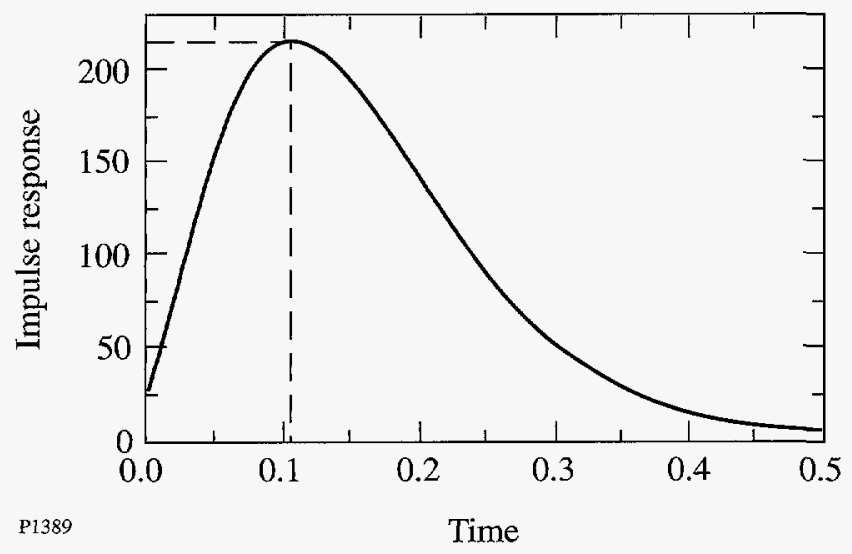

Figure 60.17

Impulse response of the Stokes wave at its exit boundary $x=l[\mathrm{Eqs}$. (28) and (29)] plotted as a function of time for the case in which $\alpha_{1} l=0, \alpha_{2} l=52$, and $\gamma l=17$. Time is measured in units of the ion-acoustic transit time $l / v_{2 x}$.

$$
I_{1}(z) \sim \exp (z) /(2 \pi z)^{1 / 2}
$$

as $z \rightarrow \infty$, one can see that

$$
\begin{array}{r}
G_{11}(x, t) \propto \exp \left(\frac{2 \gamma_{0}\left[\left(x+v_{2 x} t\right)\left(v_{1 x} t-x\right)\right]^{1 / 2}}{v_{1 x}+v_{2 x}}\right. \\
\left.-\frac{v_{1}\left(x+v_{2 x} t\right)}{v_{1 x}+v_{2 x}}-\frac{v_{2}\left(v_{1 x} t-x\right)}{v_{1 x}+v_{2 x}}\right)
\end{array}
$$

for large values of $t$. Let $\psi(x, t)$ be the exponent in Eq. (31). Then $t_{*}$, the time of maximal growth, is found from the condition

$$
\partial_{t}[\psi(l, t)]_{t_{*}}=0
$$

It is not difficult to show that

$$
t_{*}=\frac{\left(\xi_{2}+\xi_{1}\right) \alpha_{s}-\left(\xi_{2}-\xi_{1}\right)\left(\alpha_{s}^{2}-\gamma^{2}\right)^{1 / 2}}{2\left(\alpha_{s}^{2}-\gamma^{2}\right)^{1 / 2}}
$$

where

$$
\xi_{1}=l / v_{1 x}, \quad \xi_{2}=l / v_{2 x}
$$

It can also be shown that

$$
B_{1}\left(l, t_{*}\right) \propto \exp \left[\left(\alpha_{d}-\beta\right) l\right]
$$

in keeping with Eqs. (23)-(25).

The preceding analysis is consistent with previous analyses in the plasma physics literature, ${ }^{4}$ in which Fourier analysis was used to determine the impulse response function $\gamma\left(v_{x}\right)$, defined as the time-asymptotic temporal growth rate of the portion of the impulse response that convects with $x$ velocity $v_{x}$. It follows from Eq. (31) that

$$
\lim _{t \rightarrow \infty} G_{11}\left(v_{x} t, t\right) \propto \exp \left[\gamma\left(v_{x}\right) t\right]
$$


where

$$
\begin{aligned}
\gamma\left(v_{x}\right) & =\frac{2 \gamma_{0}\left[\left(v_{x}+v_{2 x}\right)\left(v_{1 x}-v_{x}\right)\right]^{1 / 2}}{v_{1 x}+v_{2 x}} \\
& -\frac{v_{1}\left(v_{x}+v_{2 x}\right)}{v_{1 x}+v_{2 x}}-\frac{v_{2}\left(v_{1 x}-v_{x}\right)}{v_{1 x}+v_{2 x}} .
\end{aligned}
$$

The impulse response function $\gamma\left(v_{x}\right)$ is displayed in Fig. 60.18. Consider the portion of the impulse response that convects with velocity $v_{x}$. This portion of the impulse response travels a distance $l$ in a time $l / v_{x}$, at which point it has grown by a factor proportional to $\exp \left[\gamma\left(v_{x}\right) l / v_{x}\right]$. Thus, $\gamma\left(v_{x}\right) / v_{x}$ is the spatial growth rate of this portion of the impulse response. In the figure this spatial growth rate is the slope of a straight line from the origin to the point $\left(v_{x}, \gamma\left(v_{x}\right)\right)$ on the impulse response curve. It follows from the figure that the amplitude of the impulse response at $x=l$ increases with time until $t_{*}=l / v_{*}$, where $v_{*}$, the velocity of maximal spatial gain, is found from the condition

$$
\frac{d}{d v_{x}}\left[\frac{\gamma\left(v_{x}\right)}{v_{x}}\right]_{v_{*}}=0
$$

Subsequently, the amplitude of the impulse response decreases with time. It is clear physically that definition (38) is identical

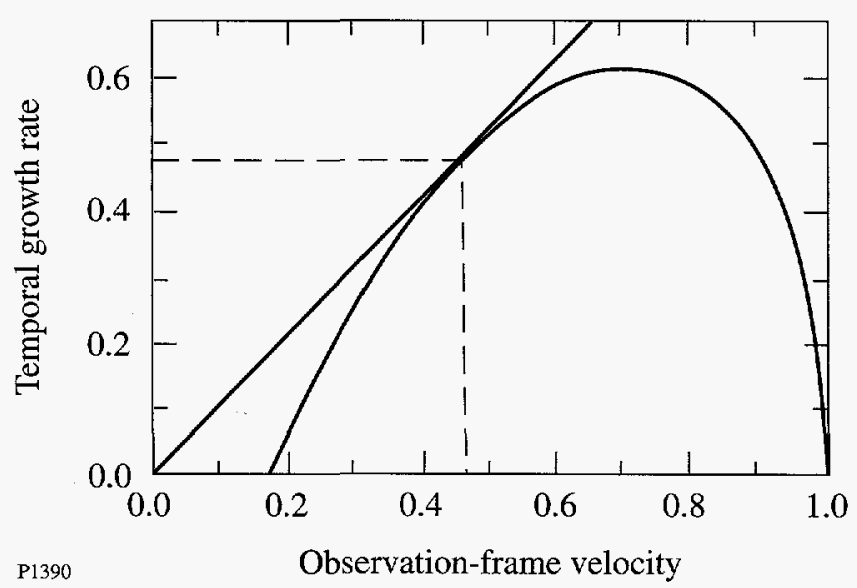

Figure 60.18

Sketch of the apparent temporal growth rate of the impulse response of the Stokes wave [Eq. (37)]. The temporal growth rate is measured in units of $\gamma_{0}$, and the observation-frame velocity is measured in units of $v_{1 x}$. to definition (32). To establish their equivalence mathematically, one need only observe that, with $x$ fixed,

$$
\frac{d}{d v_{x}}\left[\frac{\gamma\left(v_{x}\right)}{v_{x}}\right]=\frac{d}{d(x / t)}\left[\frac{\gamma(x / t) t}{x}\right]=-\frac{t^{2}}{x^{2}} \frac{\partial \psi}{\partial t}
$$

Since $v_{2} \ll v_{1}, \xi_{1} \ll \xi_{2}$ for all scattering angles, and it follows from Eqs. (15) and (33) that $t_{s}$ is proportional to $l / \sin \phi .^{11}$ This result verifies the statement that the saturation time tends to infinity as the scattering angle tends to zero. The angular dependence of the saturation time is displayed in Fig. 60.19(a). For a scattering angle of $30^{\circ}$, the saturation time is longer than that for directly backward scattering by a factor of approximately 4 . For a scattering angle of $10^{\circ}$, this factor is approximately equal to 11 . Typically, $\alpha_{1} \ll \alpha_{2}$, and it follows from Eq. (33) that

$$
\frac{v_{2 x} t_{s}}{l} \approx \frac{\alpha_{2}-\left(\alpha_{2}^{2}-4 \gamma^{2}\right)^{1 / 2}}{\left(\alpha_{2}^{2}-4 \gamma^{2}\right)^{1 / 2}} .
$$

The coefficient of the saturation time appearing on the right side of Eq. (40) is independent of the plasma length and the scattering angle. It is plotted as a function of $\gamma l$ in Fig. 60.19(b) for the case in which $\alpha_{2} l=52$. Provided that the value of $\gamma$ is not too close to its absolute threshold value of $\alpha_{2} / 2$, the saturation time is much less than the transit time of the ionacoustic wave, and our use of the infinite-medium Green function seems reasonable. As a further check of the validity of this approximation, Eqs. (18) were solved numerically for a finite plasma by a computer code based on the method of characteristics. The numerically determined Stokes output is plotted as a function of time in Fig. 60.20 for the case in which $\alpha_{2} l=52$ and $\gamma l=17$. The saturation time of $0.30 l / v_{2 x}$ predicted by Eq. (40) is consistent with the numerically determined Stokes output displayed in Fig. 60.20. The coefficient of the saturation time is also plotted as a function of the electron density and temperature in Figs. 60.21(a) and 60.21(b), respectively. The assumption that backward SBS grows and saturates convectively in a time that is short compared to the duration of the laser pulse is valid for the parameters chosen to illustrate the results of this article. [See the description of Fig. 60.15 that follows Eq. (22).] However, if the plasma length, the background electron density, or the laser intensity is significantly longer, or higher, than the value chosen for this article, or the electron temperature is significantly lower, backward SBS does not saturate and the steady-state results of the section on steady-state amplification do not apply. In this 

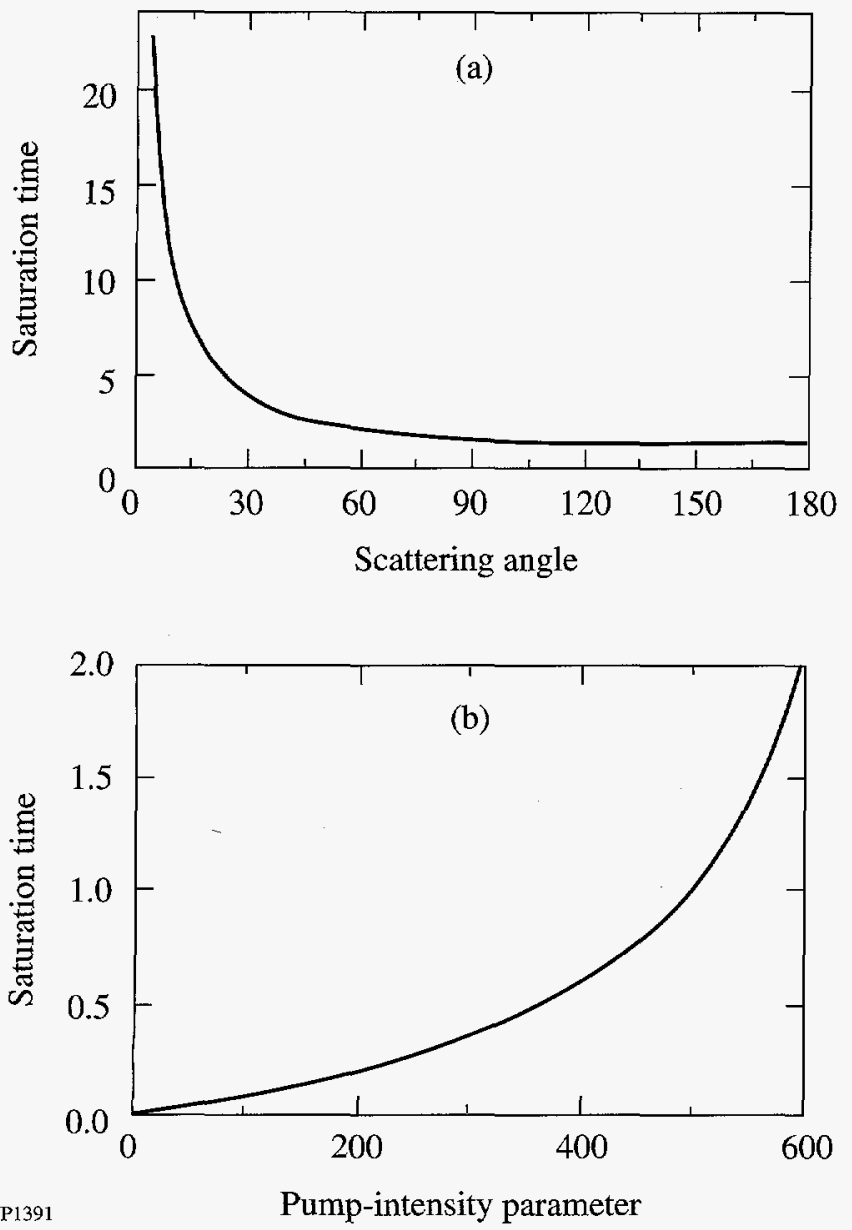

Figure 60.19

Characteristics of the convective saturation time of SBS [Eq. (40)]. (a) The saturation time, normalized to the saturation time of backward SBS, is plotted as a function of the scattering angle $2 \phi$. (b) The saturation time of backward SBS, normalized to the transit time of the ion-acoustic wave, is plotted as a function of the pump-intensity parameter $(\gamma l)^{2}$.

case, a detailed spatiotemporal analysis of $\operatorname{SBS}^{12,13}$ is required. In the strong damping regime typical of nonlinear optics experiments, Eq. (40) reduces to

$$
v_{2} t_{s} \approx 2 \gamma_{b}^{2} l / v_{1} v_{b}
$$

The saturation time is still proportional to $l / \sin \phi$, as it must be, but is independent of the acoustic speed.

As discussed in the preceding paragraph, the saturation time associated with the one-dimensional model described herein tends to infinity as the scattering angle tends to zero. It is reasonable to conclude that, at any instant of time, the Stokes output decreases as the scattering angle decreases. However, a detailed spatiotemporal analysis of SBS in a finite plasma ${ }^{12,13}$ is required to quantify the angular dependence of the Stokes output. Furthermore, as the scattering angle decreases and the one-dimensional saturation time increases, other saturation mechanisms for SBS become more important. In the context of linear theory, the only other saturation mechanism is the convection of the ion-acoustic wave in the $y$ direction. McKinstrie et al. ${ }^{14}$ have shown that this lateral convection of the ion-acoustic wave can saturate forward SBS on a time scale short compared to the one-dimensional saturation time and that the importance of lateral convection increases as the scattering angle decreases. However, as early saturation reduces the Stokes output, the general conclusion of this article-that the Stokes output decreases as the scattering angle decreases-is still correct.

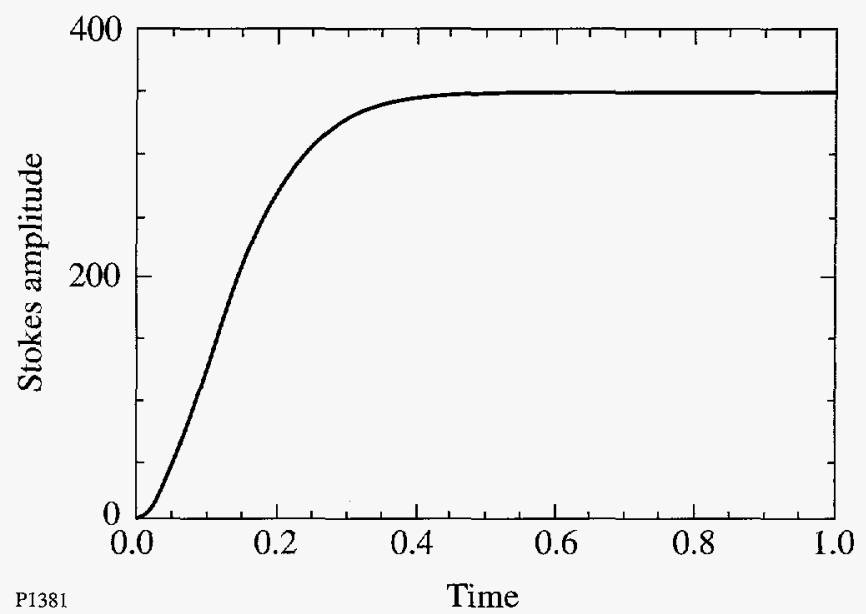

Figure 60.20

Stokes amplitude at $x=l$ plotted as a function of time for the case in which $\alpha_{1} l$ $=0, \alpha_{2} l=52$, and $\gamma l=17$. Time is measured in units of the ion-acoustic transit time. The evolution of the Stokes wave was determined by solving Eqs. (18) numerically for a finite plasma. The convective saturation time observed in this simulation is consistent with the prediction of Eq. (40).

Finally, it should be mentioned that numerical simulations of SBS in homogeneous plasma have been made by Amin et al. ${ }^{15,16}$ for the complimentary case in which the ion-acoustic wave is subject to viscous damping. In principle, the preceding analysis applies also to this case; however, the second of Eqs. (14) should read $v_{2}=v_{b} \sin ^{2} \phi$. The consequences of this modified angular dependence for the one-dimensional model described herein are profound: First, forward SBS is absolutely unstable for arbitrary laser intensity. Second, even if the laser intensity is low enough that sideward and backward SBS are not absolutely unstable, the convective saturation time for 

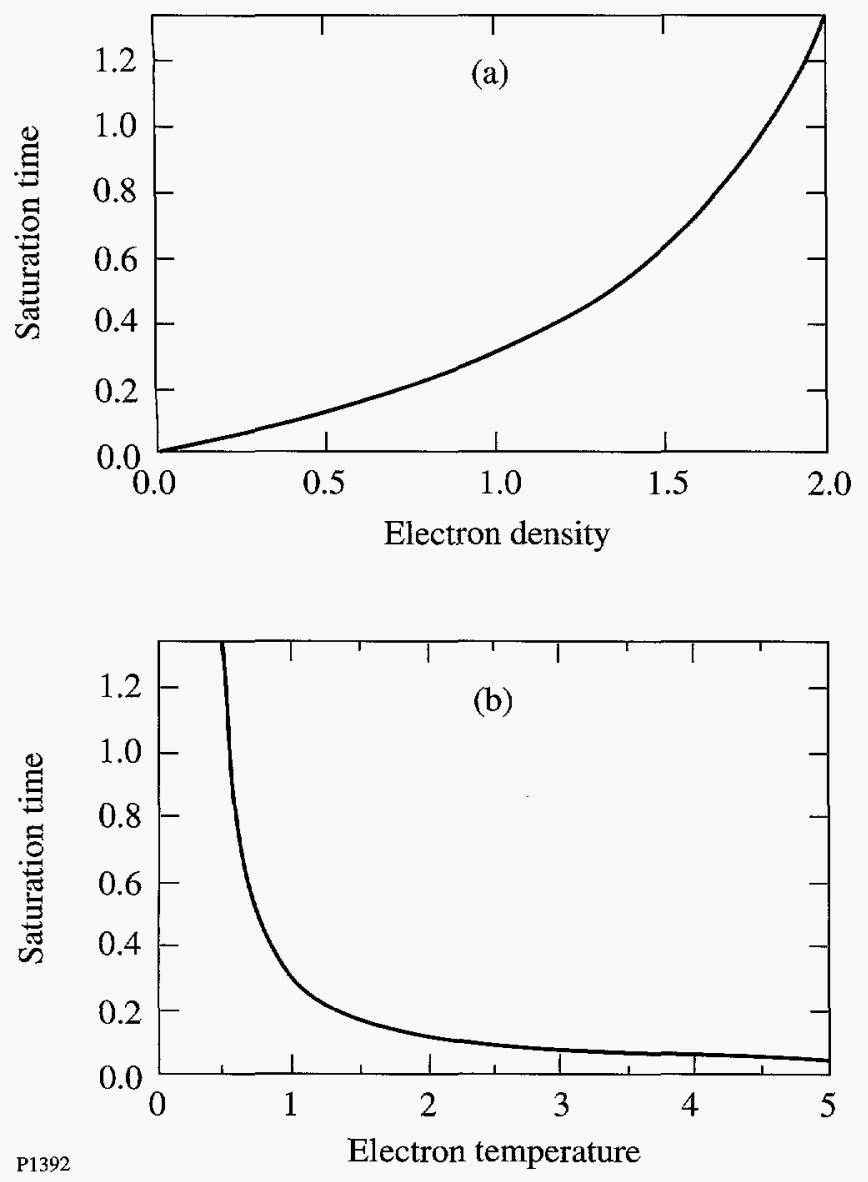

Figure 60.21

Characteristics of the convective saturation time of SBS [Eq. (40)]. (a) The saturation time, normalized to the transit time of the ion-acoustic wave, is plotted as a function of the background electron density, in units of $1019 / \mathrm{cm}^{3}$, for an electron temperature of $1 \mathrm{keV}$. (b) The saturation time, normalized to the transit time of the ion-acoustic wave, is plotted as a function of the electron temperature, in $\mathrm{keV}$, for a background electron density of $1019 / \mathrm{cm}^{3}$. In both cases the ratio of the ion and electron temperatures is 0.1 , and the laser intensity is $5 \times 10^{14} \mathrm{~W} / \mathrm{cm}^{2}$.

sideward SBS is likely to be comparable to the temporal pulsewidth of the laser. In both cases a transient two-dimensional analysis of $\mathrm{SBS}^{14}$ is required.

\section{Summary}

The angular dependence of stimulated Brillouin scattering (SBS) in a finite homogeneous plasma was studied. For parameters typical of current ICF experiments, the initial evolution of SBS is well approximated by a one-dimensional model. In the context of this model, the threshold intensity of the absolute instability and the steady-state spatial growth rate of the convective instability are both independent of the scattering angle. However, the saturation time of the convective instability exhibits a strong inverse dependence on the scattering angle: Forward SBS always occurs in the transient regime, and the intensity of the scattered light is less than that predicted by a steady-state analysis. In particular, no light is emitted in the direction parallel to the wave vector of the incident wave. Thus, the commonly held belief that backward SBS should dominate experiments involving long-scale-length plasmas is correct, but for reasons other than that on which it was originally based.

Finally, although the analysis of this article was directed at SBS, Eqs. (7) apply to other parametric instabilities such as stimulated Raman scattering (SRS). Thus, subject to the constraints described in the previous section, the main analytical results of this article should be valid for SRS.

\section{ACKNOWLEDGMENT}

This work was supported by the National Science Foundation under Contract No. PHY-9057093, the U. S. Department of Energy (DOE) Office of Inertial Confinement Fusion under Cooperative Agreement No. DE-FC0392SF19460, the University of Rochester, and the New York State Energy Research and Development Authority. The support of DOE does not constitute an endorsement by DOE of the views expressed in this article.

\section{Appendix: Green-Function Analysis of SBS in an Infinite Plasma}

The wave amplitudes $B_{1}$ and $B_{2}$ defined in the section on governing equations evolve according to the equations

$$
\begin{aligned}
& \left(\partial_{t}+v_{1} \partial_{x}+v_{1}\right) B_{1}=\gamma_{0} B_{2}+S_{1}(x, t), \\
& \left(\partial_{t}-v_{2} \partial_{x}+v_{2}\right) B_{2}=\gamma_{0} B_{1}+S_{2}(x, t),
\end{aligned}
$$

where $S_{1}$ and $S_{2}$ are phenomenological source terms that model the way in which SBS is initiated. Equations (A1) can be rewritten in the matrix form

$$
L B=S,
$$

where

$$
L=\left[\begin{array}{cc}
\left(\partial_{t}+v_{1} \partial_{x}+v_{1}\right) & -\gamma_{0} \\
-\gamma_{0} & \left(\partial_{t}-v_{2} \partial_{x}+v_{2}\right)
\end{array}\right]
$$

and

$$
B=\left[\begin{array}{l}
B_{1}(x, t) \\
B_{2}(x, t)
\end{array}\right], \quad S=\left[\begin{array}{l}
S_{1}(x, t) \\
S_{2}(x, t)
\end{array}\right] .
$$


The Green matrix

$$
G=\left[\begin{array}{ll}
G_{11}(x, t) & G_{12}(x, t) \\
G_{21}(x, t) & G_{22}(x, t)
\end{array}\right]
$$

satisfies the related equation

$$
L G=D,
$$

where

$$
D=\left[\begin{array}{cc}
\delta(x) \delta(t) & 0 \\
0 & \delta(x) \delta(t)
\end{array}\right]
$$

It is not difficult to verify that the solution of Eq. (A2) is

$$
B(x, t)=\int_{-\infty}^{\infty} \int_{-\infty}^{\infty} G\left(x-x^{\prime}, t-t^{\prime}\right) S\left(x^{\prime}, t^{\prime}\right) d x^{\prime} d t^{\prime}
$$

It follows from Eq. (A8) that the Green function $G_{i j}\left(x-x^{\prime}, t-t^{\prime}\right)$ describes the effect on wave $i$ at the position $(x, t)$ of an impulse applied to wave $j$ at the position $\left(x^{\prime}, t^{\prime}\right)$.

It is well known that the Green functions defined by Eqs. (A5)-(A7) can be written in terms of modified Bessel functions. However, as we are unaware of any derivation of this result published in the plasma physics literature, a simple derivation is included in this appendix for the benefit of the reader.

The determination of the Green matrix is facilitated by rewriting Eq. (A6) in terms of the characteristic variables

$$
\xi=x+v_{2} t, \eta=v_{1} t-x .
$$

By using these variables and the fact that

$$
\delta(x) \delta(t)=\delta(\xi) \delta(\eta) J(\xi, \eta ; x, t)
$$

where

$$
J(\xi, \eta ; x, t)=v_{1}+v_{2}
$$

is the Jacobian determinant associated with the transformation from $(\xi, \eta)$ to $(x, t)$, one can rewrite Eq. (A6) as

$$
\left[\begin{array}{cc}
\left(\partial_{\xi}+\alpha_{1}\right) & -\gamma \\
-\gamma & \left(\partial_{\eta}+\alpha_{2}\right)
\end{array}\right]\left[\begin{array}{cc}
G_{11} & G_{12} \\
G_{21} & G_{22}
\end{array}\right]
$$

$$
=\left[\begin{array}{cc}
\delta(\xi) \delta(\eta) & 0 \\
0 & \delta(\xi) \delta(\eta)
\end{array}\right]
$$

where

$$
\begin{aligned}
& \alpha_{1}=v_{1} /\left(v_{1}+v_{2}\right), \\
& \alpha_{2}=v_{2} /\left(v_{1}+v_{2}\right), \\
& \gamma=\gamma_{0} /\left(v_{1}+v_{2}\right) .
\end{aligned}
$$

One can eliminate the damping terms $\alpha_{1}$ and $\alpha_{2}$ by defining

$$
G(\xi, \eta)=\bar{G}(\xi, \eta) \exp \left(-\alpha_{1} \xi-\alpha_{2} \eta\right)
$$

The Green matrix $\bar{G}(\xi, \eta)$ satisfies the conservative equation

$$
\left[\begin{array}{cc}
\partial_{\xi} & -\gamma \\
-\gamma & \partial_{\eta}
\end{array}\right]\left[\begin{array}{ll}
\bar{G}_{11} & \bar{G}_{12} \\
\bar{G}_{21} & \bar{G}_{22}
\end{array}\right]=\left[\begin{array}{cc}
\delta(\xi) \delta(\eta) & 0 \\
0 & \delta(\xi) \delta(\eta)
\end{array}\right],
$$

which is the simplest form of Eq. (A6).

The Green functions $\bar{G}_{11}(\xi, \eta)$ and $\bar{G}_{21}(\xi, \eta)$ satisfy the equations

$$
\partial_{\xi} \bar{G}_{11}=\gamma \bar{G}_{21}+\delta(\xi) \delta(\eta), \partial_{\eta} \bar{G}_{21}=\gamma \bar{G}_{11}
$$

It follows from Eqs. (A16) and the theory of characteristics that $\bar{G}_{11}(\xi, \eta)$ and $\bar{G}_{21}(\xi, \eta)$ can only be nonzero for $\xi \geq 0$ and $\eta \geq 0$. In other words, one has to solve an initial value problem in the retarded time variable $\eta$ on the half-space $\xi \geq 0$. A natural way to solve such a problem is by Laplace transforming in the variable $\eta$. It is not difficult to show that

$$
\begin{aligned}
\bar{G}_{21}(\xi, \eta) & =\mathcal{L}^{-1}\left\{\frac{\gamma \exp \left(\gamma^{2} \xi / s\right)}{s}\right\} \\
& =\gamma I_{0}\left[2 \gamma(\xi \eta)^{1 / 2}\right] H(\xi) H(\eta) .
\end{aligned}
$$


The Laplace transform tables of Abramowitz and Stegun ${ }^{17}$ were used to invert the Laplace transform, and the Heaviside step functions $H(\xi)$ and $H(\eta)$ were added to ensure that the Green function equals zero for points outside the domain of influence of the source point. Similarly,

$$
\begin{aligned}
\bar{G}_{11}(\xi, \eta) & =\mathcal{L}^{-1}\left\{s \frac{\exp \left(\gamma^{2} \xi / s\right)}{s}-1+1\right\} \\
& =\partial_{\eta} I_{0}\left[2 \gamma(\xi \eta)^{1 / 2}\right]+\delta(\eta) \\
& =\gamma(\xi / \eta)^{1 / 2} I_{1}\left[2 \gamma(\xi \eta)^{1 / 2}\right] H(\xi) H(\eta)+H(\xi) \delta(\eta)
\end{aligned}
$$

Notice that $\bar{G}_{11}(\xi, \eta)$ is related to $\bar{G}_{21}(\xi, \eta)$ by the second of Eqs. (A16), as it must be. It follows from Eq. (A15) that $\bar{G}_{12}(\xi, \eta)=\bar{G}_{21}(\eta, \xi)$ and $\bar{G}_{22}(\xi, \eta)=\bar{G}_{11}(\eta, \xi)$.

In terms of the original variables $x$ and $t$, the conservative Green functions are

$$
\begin{aligned}
\bar{G}_{11}(x, t) & =\frac{\gamma_{0}}{v_{1}+v_{2}}\left(\frac{x+v_{2} t}{v_{1} t-x}\right)^{1 / 2} I_{1}\left(\frac{2 \gamma_{0}\left[\left(x+v_{2} t\right)\left(v_{1} t-x\right)\right]^{1 / 2}}{v_{1}+v_{2}}\right) \\
& \times H\left(x+v_{2} t\right) H\left(v_{1} t-x\right)+H\left(x+v_{2} t\right) \delta\left(v_{1} t-x\right), \\
\bar{G}_{12}(x, t) & =\bar{G}_{21}(x, t)=\frac{\gamma_{0}}{v_{1}+v_{2}} I_{0}\left(\frac{2 \gamma_{0}\left[\left(x+v_{2} t\right)\left(v_{1} t-x\right)\right]^{1 / 2}}{v_{1}+v_{2}}\right) \\
& \times H\left(x+v_{2} t\right) H\left(v_{1} t-x\right), \\
\bar{G}_{22}(x, t) & =\frac{\gamma_{0}}{v_{1}+v_{2}}\left(\frac{v_{1} t-x}{x+v_{2} t}\right)^{1 / 2} I_{1}\left(\frac{2 \gamma_{0}\left[\left(x+v_{2} t\right)\left(v_{1} t-x\right)\right]^{1 / 2}}{v_{1}+v_{2}}\right) \\
& \times H\left(x+v_{2} t\right) H\left(v_{1} t-x\right)+\delta\left(x+v_{2} t\right) H\left(v_{1} t-x\right) .
\end{aligned}
$$

According to Eq. (A14), the dissipative Green functions are related to the conservative Green functions by
$G_{i j}(x, t)=\bar{G}_{i j}(x, t) \exp \left[-\frac{v_{1}\left(x+v_{2} t\right)}{v_{1}+v_{2}}-\frac{v_{2}\left(v_{1} t-x\right)}{v_{1}+v_{2}}\right]$.

\section{REFERENCES}

1. J. F. Drake et al., Phys. Fluids 17, 778 (1974),

2. R. S. Craxton, R. L. McCrory, and J. M. Soures, Sci. Am. 255, 68 (1986).

3. C. S. Liu, in Advances in Plasma Physics, edited by A. Simon and W. B. Thompson (Wiley, New York, 1976), Vol. 6, p. 121.

4. A. Bers, in Basic Plasma Physics, edited by A. A. Galeev and R. N. Sudan, Handbook of Plasma Physics (North Holland, Amsterdam, 1983), Vol. 1, p. 45 and references therein.

5. C. J. McKinstrie and M. V. Goldman, J. Opt. Soc. Am. B 9, 1778 (1992).

6. W. L. Kruer, The Physics of Laser Plasma Interactions, Frontiers in Physics (Addison-Wesley, CA, 1988), Vol. 73, Chap. 8.

7. S. Ichimaru, Basic Principles of Plasma Physics: A Statistical Approach, Frontiers in Physics (Benjamin, Reading, MA, 1973), p. 71 .

8. R. W. Boyd, K. Rzazewski, and P. Narum, Phys. Rev. A 42, 5514 (1990).

9. C. J. McKinstrie, J. S. Li, R. Betti, and E. A. Williams, Paper 103, presented at the 23rd Annual Anomalous Conference, Wintergreen, VA, 21-25 June 1993. This work is being prepared for submittal to Physics of Plasmas.

10. C. J. McKinstrie and A. Simon, Phys. Fluids 29, 1959 (1986) and references therein.

11. C. J. McKinstrie and R. E. Giacone, Bull. Am. Phys. Soc. 37, 1440 (1992); R. E. Giacone, C. J. McKinstrie, R. Betti, and H. Chen, Bull. Am. Phys. Soc. 38, 1914 (1993).

12. D. L. Bobroff and H. A. Haus, J. Appl. Phys. 38, 390 (1967).

13. E. A. Williams and R. R. McGowan, in Research Trends in Physics: Inertial Confinement Fusion, edited by K. A. Brueckner (American Institute of Physics, New York, 1992), p. 325.

14. C. J. McKinstrie, R. Betti, R. E. Giacone, T. Kolber, and J. S. Li, Phys. Rev. E 50, 2182 (1994).

15. M. R. Amin et al., Phys. Rev. Lett. 71, 81 (1993).

16. M. R. Amin et al., Phys. Fluids B 5, 3748 (1993).

17. M. Abramowitz and I. A. Stegun, eds., Handbook of Mathematical Functions (National Bureau of Standards, Washington, D.C., 1964), Chap. 29. 


\section{Femtosecond Excited-State Dynamics of a Conjugated Ladder Polymer}

Among the known classes of nonlinear optical materials, $\pi$-conjugated polymers are very attractive because of their large, third-order optical susceptibilities and ultrafast response times. ${ }^{1-5}$ The large optical nonlinearity of conjugated polymers has long been recognized as arising from the $\pi$-electron delocalization along the polymer chains. ${ }^{2}$ Recently, aromatic heterocyclic ladder-type polymers, such as poly (benzimidazobenzophenanthroline) ladder (BBL) and semi-ladder (BBB), have received growing attention as a new class of nonlinear optical (NLO) polymers ${ }^{3-5}$ because of their desirable physical properties and their ready processibility into good opticalquality thin films. ${ }^{6}$ For example, BBL has an excellent thermal stability up to $700^{\circ} \mathrm{C}$ in a nitrogen atmosphere $\left(-650^{\circ} \mathrm{C}\right.$ in air) and has good mechanical properties in the form of films or fibers; ${ }^{6}$ both of these features suggest that the optical damage threshold may be very high. The rigid, planar, "double stranded," and quasi-two-dimensional structure of BBL (inset of Fig. 60.22) suggests maximum $\pi$-electron delocalization, leading to a large, third-order optical susceptibility $\chi^{(3)}$ as found in picosecond degenerate four-wave mixing (DFWM) ${ }^{4}$ and picosecond third-harmonic-generation (THG) experiments. ${ }^{5}$ However, the temporal resolution of the nonlinear optical response of BBL was limited to $30 \mathrm{ps}$ by the experimental equipment in both of these prior studies. 4,5

The excited-state dynamics of conjugated polymers has been extensively studied following the theoretical prediction that new intragap states can be generated by photoexcitation within an optical phonon cycle $(<100 \mathrm{fs}) .{ }^{7}$ Because of the normally strong electron-phonon coupling in conjugated polymers, self-localized excited states are formed upon photoexcitation. Examples of these structurally relaxed states include solitons, polarons, bipolarons, and excitons. ${ }^{8-10}$ These photoexcitations play a critical role in the nonlinear optical behavior of the conjugated polymers, which are often considered to be quasi-one-dimensional systems. Ultrafast transient spectroscopy studies of photoexcitations in conjugated polymers have been widely reported on single-stranded, non-ladder-type polymers such as trans-polyacetylene (PA), polydiacetylenes (PDA's), polythiophenes (PT's), poly(p-phe- nylene vinylenes) (PPV's), and poly(thiophene vinylenes) (PTV's). ${ }^{11-19}$ The unique topological features of BBL among the known conjugated polymers give rise to novel charge transport properties. ${ }^{20}$ This and the recent interest in its nonlinear optical and optoelectronic properties make this ladder polymer an excellent candidate for a detailed investigation of its ultrafast excited-state dynamics.

In this article we report studies of the time-resolved femtosecond ( $\mathrm{fs}$ ) dynamics following photoexcitation in a BBL thin film. The experimental results reveal a sub-picosecond response of the optical nonlinearity. We show that the nonlinear excitations in the ladder polymer are similar to those observed in single-stranded conjugated polymers.

The measurements were performed at room temperature on a BBL thin-film sample. The sample was spin coated on a sapphire substrate and was approximately $500 \AA$ A thick. The details of the synthesis of $\mathrm{BBL}$ and fabrication of thin films have been described elsewhere. ${ }^{6}$ An amplified colliding-pulse mode-locked (CPM) laser system was employed for the pump and probe measurements. Light pulses of approximately $100 \mathrm{fs}$ centered at $620 \mathrm{~nm}$ were generated from the CPM laser and further amplified by a copper vapor laser-pumped dye amplifier to the energy level of about $1 \mu \mathrm{J}$ per pulse at a repetition rate of $8.7 \mathrm{kHz}$. Ninety percent of the amplified pulses were focused onto an ethylene glycol jet to generate a white-light continuum that provided probe pulses tunable from $500 \mathrm{~nm}$ to $900 \mathrm{~nm}$. The remaining $10 \%$ were used as pump pulses. The intensity of the pump pulses on the sample was $\sim 1 \mathrm{GW} / \mathrm{cm}^{2}$. A cross-polatization arrangement of the pump and probe beams was used to eliminate the coherent artifact. The BBL sample exhibited excellent stability throughout the experiments, and no signal degradation was found.

Figure 60.22 shows the room-temperature linear absorption spectrum and the chemical structure of BBL. The onset of major optical absorption occurs at $690 \mathrm{~nm}(1.8 \mathrm{eV})$. The pump pulses $(2 \mathrm{eV})$ used in the experiments were well above the optical gap. The results of the pump- and continuum-probe 
experiment on BBL are shown in Fig. 60.23. The transient photoinduced probe transmission change $(\Delta T / T)$ was measured as a function of the probe time delay. For probe wavelengths above the optical gap ( $\lambda=620 \mathrm{~nm}$ and $650 \mathrm{~nm})$, increased transmission (bleaching) was observed. For probe below the gap $(\lambda>690 \mathrm{~nm})$, photoinduced absorption was observed. Both bleaching and absorption signals appear promptly (within our time resolution) following the excitation. Figure 60.24 shows the fit of the bleaching and absorption signals for three different probe wavelengths. Neither bleaching nor absorption decay can be fitted by a single exponential function. The decay of the bleaching signal is best fitted to a biexponential function and a constant term of the form $\Delta T / T=A \exp \left(-t / \tau_{1}\right)+B \exp \left(-t / \tau_{2}\right)+C$. For the $620-\mathrm{nm}$ probe, the initial fast decay has a time constant $\tau_{1}=0.39 \mathrm{ps}$, whereas the slow component is $\tau_{2}=2.11 \mathrm{ps}$. The best fit to all the transient absorption traces is a biexponential function with a fast component and a slow component of the form $\Delta T / T=A \exp \left(-t / \tau_{1}\right)+B \exp \left(-t / \tau_{2}\right)$. The decay of the photoinduced absorption is much slower than that of the photo-induced bleaching and is also wavelength dependent. The slowest decay occurs at $770 \mathrm{~nm}$ when $\tau_{1}=1.03 \mathrm{ps}$ and $\tau_{2}=$ $29.5 \mathrm{ps}$. The results of fitting the photoinduced bleaching and absorption data are summarized in Table 60.II.

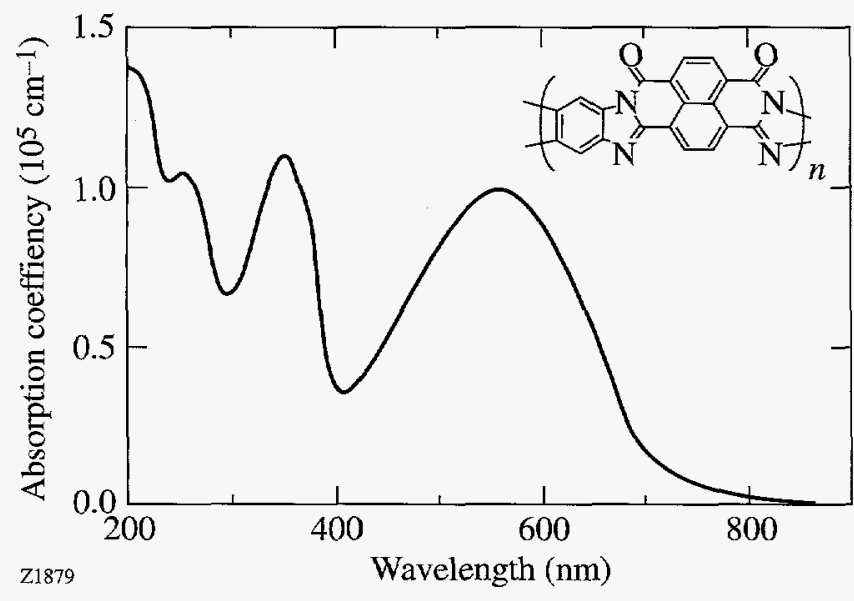

Figure 60.22

The room-temperature absorption spectrum and the chemical structure (inset) of BBL.

The ultrafast dynamics can be explained by the photogeneration and decay of self-trapped excitons (STE's) or polaron-excitons. ${ }^{16,17}$ In nondegenerated ground-state polymers, photogenerated electron-hole pairs are confined through the preferred sense of bond alternation on the polymer chain and cannot be totally separated. The main product of intra-

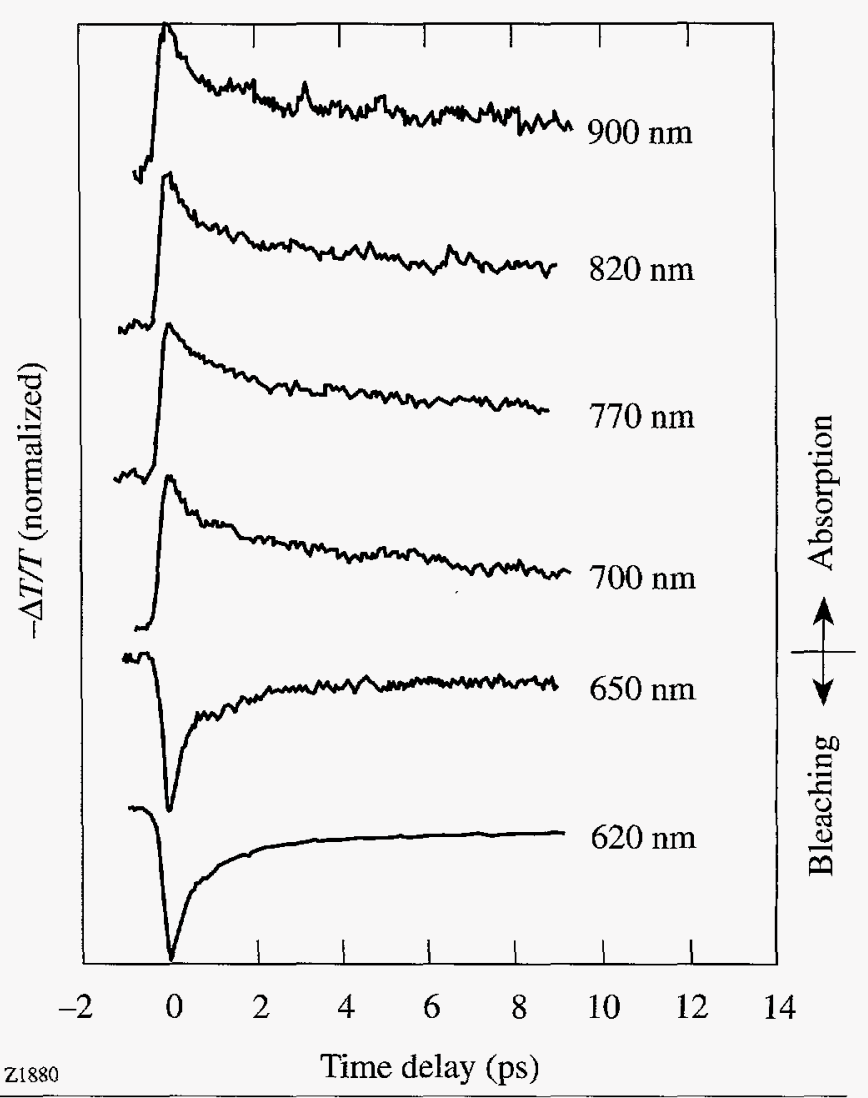

Figure 60.23

Transient photoinduced transmission change $(\Delta T / T)$ measured as a function of the probe delay time at different probe wavelengths following excitation at $620 \mathrm{~nm}$. The traces have been displaced vertically for clarity. Bleaching is observed above $E_{g}$ and induced absorption below $E_{g}$.

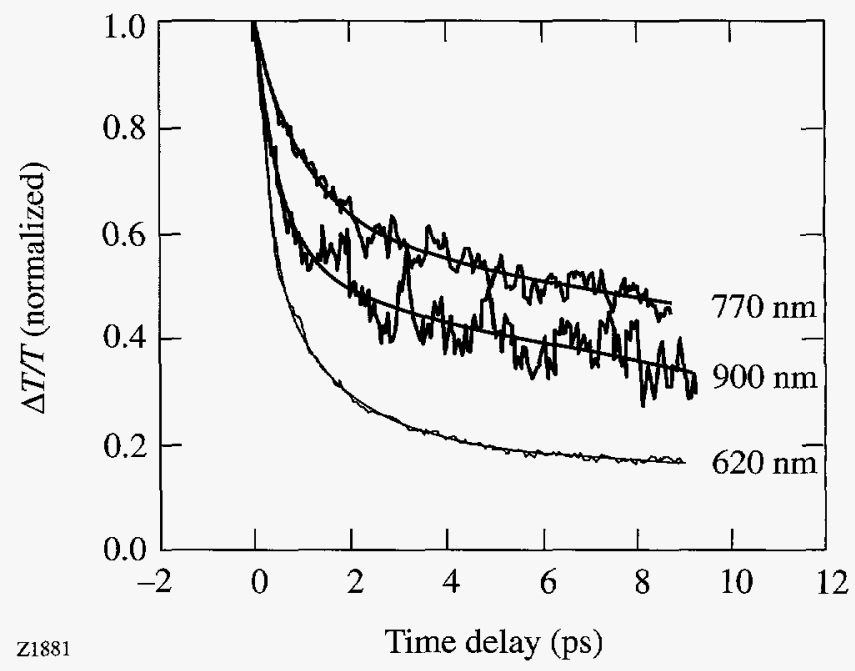

Figure 60.24

Fitting of the decay curves of the bleaching signal (probe at $620 \mathrm{~nm}$ ) and absorption signals (probe at $770 \mathrm{~nm}$ and $900 \mathrm{~nm}$ ). The smooth curves are fits as described in the text. 
Table 60.II The lifetimes resulting from fitting of the decay curves at different probing wavelengths $\lambda$.

\begin{tabular}{|c|c|c|}
\hline$\lambda(\mathrm{nm})$ & $\tau_{1}(\mathrm{ps})$ & $\tau_{2}(\mathrm{ps})$ \\
\hline 620 & $0.39 \pm 0.01$ & $2.11 \pm 0.1$ \\
\hline 650 & $0.25 \pm 0.02$ & $1.46 \pm 0.1$ \\
\hline 700 & $0.60 \pm 0.05$ & $15.3 \pm 0.5$ \\
\hline 770 & $1.03 \pm 0.06$ & $29.5 \pm 2.2$ \\
\hline 820 & $0.59 \pm 0.05$ & $18.9 \pm 1.0$ \\
\hline 900 & $0.59 \pm 0.05$ & $20.5 \pm 1.6$ \\
\hline
\end{tabular}

chain photoexcitation is neutral, self-trapped excitons. ${ }^{10}$ Kobayashi et al. studied photoexcitations in polydiacetylene and explained their results by the self-trapped exciton model. ${ }^{16,17}$ Greene et al. have studied the excitonic absorption saturation effect in polydiacetylene and explained their results by exciton phase-space filling. ${ }^{21}$ Recently, Samuel et al. have studied the picosecond photoinduced absorption in PTV and PPV and have assigned the observed signals to polaronexcitons. ${ }^{22}$ Since BBL has a nondegenerated ground state and the observed photoinduced absorption and bleaching signals in BBL resemble those in PDA and PPV, we attribute them to STE's. Other excitations such as bipolarons or triplet excitons have very little contribution to the signals within the time window (10 ps) of our experiments.

Figure 60.25 shows the adiabatic potential energy curves of the ground and excited states of the system as functions of the lattice deformation $Q$. The ground and excited states are represented by parabolic curves. The equilibrium lattice deformation $Q=0$ corresponds to a perfect dimerized lattice resulting from the Peierls instability. Free excitons are created by $\pi-\pi^{*}$ photoexcitation. Due to the strong electron-phonon coupling, free excitons are unstable and undergo self-trapping quickly after creation. Since in a quasi-one-dimensional system there is no barrier between the potential curves of the free excitons and the STE's, self-trapping takes place within the coupled phonon period ( $<100 \mathrm{fs}$ ) and produces hot STE's. ${ }^{23,24}$ The hot STE's then thermalize toward the bottom of the STE potential surface by emission of phonons. Simultaneously, the hot STE's can relax directly to the ground state. This occurs via an oscillation passing over the point where the STE potential crosses the ground state potential. ${ }^{16,17}$ The initial decay of the bleaching is due to the combination of these two processes. The thermalized excitons then decay to the ground state either

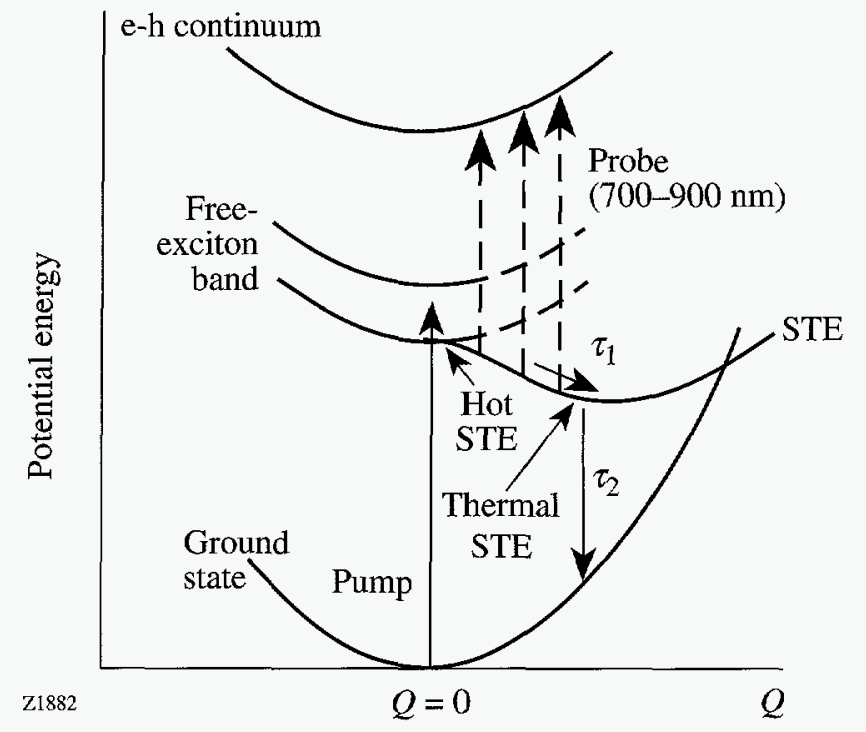

Figure 60.25

The adiabatic potential energy curves of the excited and ground states plotted against the lattice deformation $Q$.

radiatively or nonradiatively. We find that the characteristic decay time of STE's is of the order of 10 ps, which is much shorter than the expected radiative lifetime of STE's ( $1 \mathrm{~ns}$ ), indicating that the nonradiative pathways play a significant role. This is consistent with our observation of the low photoluminescence quantum yield in BBL. The nonradiative decay of STE's is considered to be either tunneling between the potential energy curves from the exciton state to the ground state or the evolution of excitons to form new intermediate species between the exciton and the ground state, such as excimers. ${ }^{25}$ There is a constant component in bleaching signals that accounts for about $20 \%$ of the maximum signal. This constant component may be associated with the formation of new species, which results in a very slow recovery of the ground-state populations.

The induced absorption is assigned to the transitions from the self-trapped excitons to higher excited continuum states. Although both the bleaching signal and the absorption signal contain a fast decay component and a slow decay component, the decay of the absorption is much slower than that of the bleaching. The decay of the photoinduced absorption is also wavelength dependent, with the slowest decay at $770 \mathrm{~nm}$. One possible reason for this can be seen by examining the thermalization and cooling of the hot STE's. The hot excitons reach the bottom of the potential curve in two steps. They thermalize first to form quasi-thermalized STE's by phonon emission. These quasi-thermalized excitons have still not reached the bottom of the potential curve; they cool to the bottom by 
coupling with phonon modes of lower frequencies. ${ }^{16}$ Thus by probing at different wavelengths, we follow the evolution of the STE's as shown in Fig. 60.25. The bleaching signal that monitors the disappearance of free excitons is due to both hot STE's and quasi-thermalized STE's. The absorption signal, however, comes mainly from thermalized or cold STE's, depending on the probing wavelength. Since the thermalized excitons are closer to the bottom of the potential curve than the hot excitons, their decay to the ground state is by tunneling through a higher and thicker potential barrier and is expected to be slower than that of hot excitons. When probing at longer wavelengths $(820 \mathrm{~nm}, 900 \mathrm{~nm})$, the signal comes from excitons that are hotter than those seen by probing at $770 \mathrm{~nm}$; therefore, the decay should be faster, in agreement with the experimental data.

In conclusion, the dynamics of the photoexcitations in the conjugated ladder polymer BBL has been studied for the first time by femtosecond time-resolved absorption measurements. Photoinduced bleaching of the $\pi-\pi^{*}$ absorption band and intragap photoinduced absorption were observed. The observed excited-state decay dynamics is consistent with the self-trapped exciton model and is tentatively explained by the generation and decay of the STE's. The observed signals arise within our time resolution and feature both a fast and a slow component. The fast component is attributed to the selftrapping and thermalization of free excitons, while the slow component was assigned to the relaxation of the quasi-thermalized and/or cold STE's to other excited state species or the ground state. The difference between the decay dynamics of bleaching and absorption has been explained by the thermalization and cooling of hot STE's.

\section{ACKNOWLEDGMENT}

This work was supported in part by the National Science Foundation (CTS 9311741) and the Center for Photoinduced Charge Transfer. Y. Kostoulas acknowledges support of the Link Foundation.

\section{REFERENCES}

1. Materials for Nonlinear Optics: Chemical Perspectives, edited by S. R. Marder, J. E. Sohn, and G. D. Stucky (American Chemical Society, Washington, DC, 1991).
2. G. P. Agrawal, C. Cojan, and C. Flytzanis, Phys. Rev. B 17, 776(1978).

3. A. K. Agrawal et al., J. Phys. Chem. 96, 2837 (1992).

4. J. R. Lindle et al., Appl. Phys. Lett. 56, 712 (1990).

5. S. A. Jenekhe et al., Mater. Res. Soc. Symp. Proc. 214, 55 (1991).

6. S. A. Jenekhe and P. O. Johnson, Macromolecules 23, 4419 (1990).

7. W. P. Su and J. R. Schrieffer, Proc. Natl. Acad. Sci. USA. 77, 5626 (1980).

8. K. Fesser, A. R. Bishop, and D. K. Campbell, Phys. Rev. B 27, 4804 (1983).

9. A. J. Heeger et al., Rev. Mod. Phys, 60, 781 (1988).

10. R. H. Friend, D. D. C. Bradley, and P. D. Townsend, J. Phys. D: Appl. Phys. 20, 1367 (1987).

11. C. V. Shank et al., Phys. Rev. Lett. 49, 1660 (1982).

12. J. Orenstein, in Handbook of Conducting Polymers, edited by T. A. Skothein (Marcel Dekker, New York, 1986), Vol. 2, p. 1297.

13. G. M. Carter et al., Appl. Phys. Lett. 49, 998 (1986).

14. B. I. Green et al., Chem. Phys. Lett. 139, 381 (1987).

15. G. J. Blanchard et al., Chem. Phys. Lett. 158, 329 (1989).

16. T. Kobayashi et al., J. Opt. Soc. Am. B 7, 1558 (1990).

17. M. Yoshizawa, A. Yasuda, and T. Kobayashi, Appl. Phys. B 53, 296 (1991).

18. J. M. Huxley et al., Appl. Phys. Lett. 56, 1600 (1990).

19. B. C. Hess et al., Phys. Rev. Lett. 66, 2364 (1991).

20. H. Mizes and E. Conwell, Phys. Rev. B 44, 3963 (1991).

21. B. I. Greene et al., Phys. Rev. Lett. 61, 325 (1988).

22. I. D. Samuel et al., Synth. Met. 55-57, 15-21 (1993).

23. E. I. Rashba and M. D. Sturge, in Excitons, edited by E. I. Rashba and M. D. Sturge (North-Holland, Amsterdam, 1982), p. 578.

24. K. Nasu, J. Lumin. 38, 90 (1987).

25. S. A. Jenekhe and J. A. Osaheni, Science 265, 765 (1994). 


\section{OMEGA Upgrade Status Report 10/1/93-9/30/94}

The design effort for the OMEGA Upgrade project was finished and the project transitioned to the manufacturing phase during the first two quarters of FY94. The manufacturing of the OMEGA Upgrade consists of fabrication, assembly and testing of all components installed into the laser system, and then integration of all subsystems into a complete facility. The status of the project is indicated by the DOE milestones completed and started during FY94.

DOE project milestones completed this fiscal year are

KD3' design

Operations readiness review, facility

Laser drivers design

Pulse-generation room activation

Target chamber installation

KD3a transition to operations (pending DOE approval)

Delivery of

- laser bay structures

- target bay structures

- disk amplifier power conditioning

DOE project milestones started this fiscal year are

Assembly of rod and disk amplifiers

Laser bay integration

Power conditioning installation

Target bay integration

Installation of laser and target structures

Target bay optomechanical assemblies (in-house mfg.)

Laser optics installation

Delivery of KDP crystals

A total of 104 mechanical structures are required to mount the optical assemblies on the OMEGA Upgrade laser facility. All of these laser and target bay structures and optical tables have been received, aligned, and grouted into their final positions. The 243 spatial filter tube sections were received, cleaned, and installed into their support frames.
The contractor delivered, assembled, and resistive load tested all 120 SSA (disk amplifier) power conditioning units (PCU's). These units are now ready for live-load testing and amplifier activation. The project received and assembled 11 of the 17 rod amplifier PCU's needed for the first three stages of amplification (stages A, B, and C), and completed mechanical assembly of all rod PCU's needed for the fourth amplification stage (stage D-the final rod amplifiers).

The hardware to transport the main driver pulse from the pulse-generation room (PGR) to the driver line (preamplifier section) was installed, tested, and activated. The main-pulse driver line construction and testing was completed and has delivered a 10-J laser pulse at the output of the driver line ready for injection into the power amplifier section of the laser. Transport from the PGR and driver line for the foot pulse is in work and is scheduled for completion by the end of FY94. These tasks include the construction and testing of large-aperture ring amplifiers (LARA's) used in both the main- and foot-pulse driver lines.

The design for the rod and disk amplifiers resulted in approximately 500,000 parts. The fabrication procurements for these parts were placed and nearly over-subscribed the local machine shops. There are 97 rod amplifiers, $6015-\mathrm{cm}$ SSA amplifiers, and $6020-\mathrm{cm}$ SSA amplifiers needed for the OMEGA Upgrade laser system. We have installed 38 rod, 15 15-cm SSA, and one 20-cm SSA amplifier assemblies into their structures on the laser bay floor.

The Thin Film Coating Group has processed through their facility 1047 of the 2430 optics required for the laser system. The remaining 1383 optics are due for delivery to the installation teams by mid-December. The optics are being supplied on an as-needed schedule to minimize the need for clean room storage space. 
For FY94 the laser bay integration alignment progress follows closely behind the installation of optical components delivered to the facility. This effort began in early March with the installation and activation of a continuous IR laser source and the optics required for a full-aperture alignment beam. Laser beamline alignment requires the installation of several alignment diagnostics, including a collimation sensor capable of measuring the system collimation at any point in the IR laser between the 243 Upgrade spatial filters. All optics are installed and aligned through the stage- $\mathrm{C}$ alignment sensor packages (ASP's) at the conclusion of FY94. The stage-C ASP is midchain alignment diagnostic installed just prior to the final beam splitters (C-D splitter). The C-D splitter is currently under alignment as are beamlines starting at the stage-D rod amp and propagating to the stage- $\mathrm{F}$ alignment sensor packages (F-ASP's). Most optics have been installed in the ten-beam cluster-3 assemblies, and alignment is progressing smoothly in this cluster.

The target bay integration alignment has concentrated on the ultraviolet alignment table (UVAT), periscope mirror assembly (PMA), and the F-ASP. These three subsystems are required to establish the baseline alignment for all optics in the laser and target bays. The UVAT is fully operational, as are the PMA's. The F-ASP's have all 60 primary and secondary grouting frames installed and aligned. The cluster- 3 F-ASP has all ten alignment relay subsystems installed and is fully supported by a control system and alignment image processing.

The activation process of a system stage closely follows the integration alignment of that stage. The driver line is fully activated and calibrated and is operational to support the daily activation requirements. Cluster-3 beamline 9 (39) was se- lected as the first line to be activated. Amplifiers A3, B34, and C34 have been test fired, fully activated, and calibrated to support the activation of this beam line. All the splitters in beam line 34 have been optically balanced with ratiometers. Full activation for beam line 39 is to be completed by midOctober. Lessons learned from beamline 39 will increase the efficiency of activation for the remaining 59 beamlines.

The project schedule called for the delivery of an Acceptance Test Plan by the end of the third quarter of FY94 and a Final Safety Analysis Document by the end of the second quarter of FY95. These are related to a final operations readiness review and the Key Decision \#4 "Project Complete Start Operations" milestone. These are embodied in two project documents that were completed and submitted to DOE in advance of the Acceptance Test Plan milestone.

The Operational Readiness Review Plan (S-AA-M-11) sets up an LLE "Operations and Safety Review Committee" that will conduct an extensive, independent review of the safety aspects of the design and operation of the OMEGA facility. The committee will prepare a report summarizing its activities and findings that will be provided to DOE in advance of the DOE Final Project review.

The Acceptance Test Plan (S-AA-M-08) sets up a system performance review process that is similar to the operations review: An LLE "System Performance Review Committee" will monitor the activation of the system and review test data to verify that the project performance baselines have been met. The committee's report will be provided to DOE in advance of the DOE Final Project review. 


\section{NLUF News}

Six NLUF proposals for FY94 were submitted to DOE for the consideration of the Steering Committee (see Table 60.III). All of the principal investigators, representing four universities and two government laboratories, have had previous proposals approved for experiments at LLE. Three of the experiments involve $\mathrm{x}$-ray or XUV spectroscopy, one is a plasma interaction experiment, one a diagnostics development, and one a target fabrication effort.

On 1 October 1993 these proposals were reviewed via conference call by the Steering Committee and Dr. James Knauer (NLUF Manager). Voting members included Dr. Michael Boyle, Dr. Phillip Goldstone, Prof. Chandrasakar Joshi, and Dr. Robert Turner. The Steering Committee evaluated the proposals for their technical merit and submitted their recommendation to the Oakland office of DOE.

DOE decided to fund all of the submitted proposals. The monies were allocated according to the technical ranking provided by the Steering Committee. These proposals were primarily directed to the development of diagnostics for the
OMEGA Upgrade laser system. Two projects that were to use the GDL laser system were either conducted at other laser systems or are awaiting the availability of the GDL system.

A brief summary of each proposal funded for FY94 follows:

\section{Proposal 180}

"Measurements of Quantum Electrodynamic Sensitive Transitions in Na-like and CU-like Ions"

Principal Investigator:

\section{J. Reader, National Institute of Standards and Technology (NIST)}

The Principal Investigator proposes to use GDL to measure QED effects in high- $Z$ ions. There are 11 proposed targets ranging in $Z$ from 47 to 92 . The GDL laser system will be used to study Na-like and $\mathrm{Cu}$-like ions of these elements. The primary diagnostic is a $2.2-\mathrm{m}$ Rowland Spectrograph owned by NIST with its own attached target chamber. The optics used for Dr. Reader's previous experiments are not usable on the current GDL system because of the increased beam diameter and energy. We do have optics available but the focusing lens assembly must be adapted to his target chamber.

Table 60.III: The numerical listing of NLUF proposals.

\begin{tabular}{|c|l|l|l|}
\hline $\begin{array}{c}\text { Proposal } \\
\text { Number }\end{array}$ & \multicolumn{1}{|c|}{ Investigator } & \multicolumn{1}{|c|}{ Institution } & \multicolumn{1}{c|}{ Proposal Title } \\
\hline 180 & J. Reader & $\begin{array}{l}\text { National Institute of Standards } \\
\text { and Technology }\end{array}$ & $\begin{array}{l}\text { Measurements of Quantum Electrodynamic Sensitive } \\
\text { Transitions in Na-like and Cu-like Ions }\end{array}$ \\
\hline 181 & J. F. Seely & Naval Research Laboratory & Normal-Incidence Multilayer Mirror X-Ray Microscope \\
\hline 182 & A. Honig & Syracuse University & $\begin{array}{l}\text { Temperature-Dependent Tensile Strength, Surface } \\
\text { Roughness Diagnostics, and Magnetic Support and } \\
\text { Positioning of Polymer ICF Shells at Temperatures } \\
\text { between 4K and 300K }\end{array}$ \\
\hline 183 & K. Mizuno & $\begin{array}{l}\text { Plasma Physics Research } \\
\text { Institute } \\
\text { University of California, Davis }\end{array}$ & $\begin{array}{l}\text { The Ion Acoustic Decay Instability in a Large-Scale, } \\
\text { Hot Plasma Relevant to Direct-Drive Laser } \\
\text { Fusion-Applications to a Critical Surface Diagnostic } \\
\text { and Thermal Smoothing }\end{array}$ \\
\hline 184 & C. F. Hooper Jr. & $\begin{array}{l}\text { University of Florida } \\
\text { Plasma Spectroscopy: Theoretical and Experimental } \\
\text { Diagnostic Development / Tests }\end{array}$ \\
\hline 185 & H. R. Griem & University of Maryland & $\begin{array}{l}\text { Development of Density and Temperature Profile } \\
\text { Diagnostics for ICF Targets }\end{array}$ \\
\hline
\end{tabular}


Proposal 181

"Normal-Incidence Multilayer Mirror X-Ray Microscope"

Principal Investigator:

\section{J. F. Seely, Naval Research Laboratory (NRL)}

Dr. Seeley's group at NRL is building a Cassegrain-type microscope for use at a wavelength of $33.7 \AA$. The work builds upon the successful fielding of a similar instrument on the OMEGA laser system in 1992 and development work supported by NASA. The microscope will have a $1.8-\mu$ m resolution at the target and a magnification of 10 , and will use a $1024 \times$ $1024 \mathrm{CCD}$ as the detector. Most of the work is being done at NRL. The microscope fits into a LLNL SIM cart, allowing it to be tested on either GDL or NOVA.

\section{Proposal 182}

"Temperature-Dependent Tensile Strength, Surface Roughness Diagnostics, and Magnetic Support and Positioning of Polymer ICF Shells at Temperatures between $4 \mathrm{~K}$ and $300 \mathrm{~K} "$

\section{Principal Investigator:}

\section{A. Honig, Syracuse University}

There are three independent tasks to this proposal for work to be done at Syracuse University. The first task will study the material properties of $\mathrm{CH}$ shells at cryogenic temperatures; the second task will use the measured shell properties with precharacterized shells to determine if there is a correlation between accommodation coefficients and surface roughness; and the third task will develop the use of ferrite-doped plastic shells for magnetic levitation and positioning. Ferrite-doped plastics have been delivered to LLE for shell fabrication.

\section{Proposal 183}

"The Ion Acoustic Decay Instability in a Large-Scale, Hot Plasma Relevant to Direct-Drive Laser Fusion-Applications to a Critical Surface Diagnostic and Thermal Smoothing" Principal Investigator:

\section{K. Mizuno, Plasma Physics Research Institute (UCD)}

This group is studying the critical surface using the ionacoustic decay instability. The development of this diagnostic for the OMEGA Upgrade is being done at PPRI; subsequent testing of thermal-smoothing techniques is to be done on GDL. There is a second task that will use the GDL facility to test the second-harmonic diagnostic and $\mathrm{x}$ rays emitted from flat targets to study thermal smoothing and lateral heat transport. This second task requires that GDL have full pulse shaping and smoothing by spectral dispersion capability. The GDL tasks have been delayed until the laser system is available for experiments.

\section{Proposal 184}

"Plasma Spectroscopy: Theoretical and Experimental Diagnostic Development/Tests"

\section{Principal Investigator:}

\section{F. Hooper Jr., University of Florida}

The Principal Investigator continues to develop atomic physics computer codes to calculate the effects of high temperature and density on $\mathrm{x}$-ray line emission and transport. There are three tasks associated with this work. The analysis of $L$-shell spectra is considered (by the Principal Investigator) the next step in the extension of the atomic models with ion dynamics formalism. It is also proposed to extend the "multielectron line broadening theory" to conditions expected for OMEGA Upgrade target implosions. The third task is to develop micro-dot spectroscopy to study laserplasma interactions.

\section{Proposal 185}

"Development of Density and Temperature Profile Diagnostics for ICF Targets"

\section{Principal Investigator:}

\section{H. R. Griem, University of Maryland}

The work done primarily at the University of Maryland is to extend the development of previous work in x-ray and XUV spectroscopy to OMEGA Upgrade target conditions. The diagnostics are being constructed and tested before the experiments can be done on the OMEGA Upgrade. The Trident laser system at the Los Alamos National Laboratory is being used to test the instrumentation. 


\section{PUBLICATIONS AND CONFERENCE PRESENTATIONS}

\section{Publications}

M. J. Cumbo and S. D. Jacobs, "Determination of NearSurface Forces in Optical Polishing Using Atomic Force Microscopy," Nanotechnology 5, 70 (1994).

J. Delettrez, D. K. Bradley, and C. P. Verdon, "The Role of the Rayleigh-Taylor Instability in Laser-Driven Burnthrough Experiments," Phys. Plasmas 1, 2342 (1994).

E. M. Epperlein and R. W. Short, "Generalized Electron Fluid Equations in the Presence of Laser Irradiation," Phys. Plasmas 1, 3003 (1994).

E. M. Epperlein and R. W. Short, "Nonlocal Electron Transport in the Presence of High-Intensity Laser Irradiation," Phys. Rev. E 50, 1697 (1994).

D. Gupta, W. R. Donaldson, and A. M. Kadin, "A LaserTriggered, Inductive Opening Switch Using High-Temperature Superconducting Thin Films," in Advances in Cryogenic Engineering, edited by P. Kittel (Plenum Press, New York, 1994), Vol. 39B, pp. 2015-2020.

J. W. Herman and H. E. Elsayed-Ali, "Time-Resolved Structural Studies of the Low-Index Faces of Lead," Phys. Rev. B 49, 4886 (1994).

B. S. W. Kuo and A. W. Schmid, "Effects of Thin-Film Thermal Conductivity on the Optical Damage Threshold of a-Si Film on c-Si Substrate at 1064 nm," J. Appl. Phys. 74, 5159 (1993).

P. W. McKenty, S. Skupsky, J. H. Kelly, and C. T. Cotton, "Numerical Investigation of the Self-Focusing of Broad-
Bandwidth Laser Light with Applied Angular Dispersion," J. Appl. Phys. 76, 2027 (1994).

C. J. McKinstrie, R. Betti, R. E. Giacone, T. Kolber, and J. S. Li, "Two-Dimensional Stimulated Brillouin Scattering," Phys. Rev. E 50, 2182 (1994).

S. Papernov and A. W. Schmid, "Atomic Force Microscopy Studies of Laser-Triggered Morphology Changes in $\mathrm{Y}_{2} \mathrm{O}_{3}$ Monolayer Coatings," in Laser-Induced Damage in Optical Materials: 1993 (SPIE, Bellingham, WA, 1994), Vol. 2114, pp. 381-386.

J. Peatross, J. L. Chaloupka, and D. D. Meyerhofer, "HighOrder Harmonic Generation with an Annular Laser Beam," Opt. Lett. 19, 942 (1994).

C. J. Twomey, S.-H. Chen, T. Blanton, A. W. Schmid, and K. L. Marshall, "Solid Polymers Doped with Rare Earth Metal Salts. II. Thermal Behavior and Morphology of the Neodymium Acetate-Poly(Ethylene Oxide) System," J. Polym. Sci. B, Polym. Phys. Ed. 31, 647 (1993).

C.-C Wang, M. Currie, S. Alexandrou, and T. Y. Hsiang, "Ultrafast, All-Silicon Light Modulator," Opt. Lett. 19, 1453 (1994).

W. Xiong, W. Kula, R. Sobolewski, and J. R. Gavaler, "Laser Writing: A New Technique for Fabrication of Electronic and Optoelectronic Y-Ba-Cu-O Devices and Circuits," in Superconductive Devices and Circuits, edited by R. A. Buhrman, J. Clarke, K. Daly, R. H. Koch, J. A. Luine, and R. W. Simon (SPIE, Bellingham, WA, 1994), Vol. 2160, pp. 16-24. 


\section{Forthcoming Publications}

U. Alon, J. Hecht, D. Ofer, and D. Shvarts, "Power Laws and Similarity of Rayleigh-Taylor and Richtmyer-Meshkov Mixing Fronts at All Density Ratios," to be published in Physical Review Letters.

S. Augst and D. D. Meyerhofer, "Field Ionization of Noble Gas Atoms with a Keldysh Adiabaticity Parameter of One," to be published in Laser Physics.

R. Betti, V. Goncharov, R. L. McCrory, E. Turano, and C. P. Verdon, "Multiple Cutoff Wave Numbers of the Ablative Rayleigh-Taylor Instability," to be published in Physical Review Letters.

T. R. Boehly, R. S. Craxton, T. H. Hinterman, J. H. Kelly, T. J. Kessler, S. A. Kumpan, S. A. Letzring, R. L. McCrory, S. F. B. Morse, W. Seka, S. Skupsky, J. M. Soures, and C. P. Verdon, "The Upgrade to the OMEGA Laser System," to be published in the Review of Scientific Instruments.

T. R. Boehly, R. S. Craxton, T. H. Hinterman, P. A. Jaanimagi, J. H. Kelly, T. J. Kessler, R. L. Kremens, S. A. Kumpan, S. A. Letzring, R. L. McCrory, S. F. B. Morse, W. Seka, S. Skupsky, J. M. Soures, and C. P. Verdon, "The Upgrade to the OMEGA Laser System," to be published in the Proceedings of the American Nuclear Society.

D. K. Bradley, P. M. Bell, O. L. Landen, J. D. Kilkenny, and J. Oertel, "Development and Characterization of a Pair of 30-40 ps X-Ray Framing Cameras," to be published in the Review of Scientific Instruments.

X. D. Cao, D. D. Meyerhofer, and G. P. Agrawal, "Optimization of Optical Beam Steering in Nonlinear Kerr Media Via Spatial Phase Modulation," to be published in the Journal of the Optical Society of America B.

X. D. Cao, L. Zheng, and D. D. Meyerhofer, "A Novel Method for the Measurement of Temporal Walk-Off of Short Pulses in Nonlinear Crystals," to be published in Optics Letters.

$\checkmark$ X. D. Cao and D. D. Meyerhofer, "All-Optical Switching Via Collisions of Spatial Vector Solitons," to be published in Optics Letters.
X. D. Cao and D. D. Meyerhofer, "Frequency-Domain Interferometer for Measurement of the Polarization Modal Dispersion in Single-Mode Optical Fibers," to be published in Optics Letters.

S. H. Chen, "Novel Low Molar Mass Glass-Forming Liquid Crystals: Synthesis, Characterization, and Morphological Stability," to be published in the Proceedings of the Society of Polymer Science, Japan.

S. H. Chen and S. Krishnamurthy, "Some Fundamental Issues Governing Thermotropic Chiral Nematic Copolymers," to be published in the Proceedings of the 42nd Society for Polymer Science, Kyoto, Japan, 31 May-2 June 1993.

C. Y. Chien, G. Korn, J. S. Coe, J. Squier, G. Mourou, and R. S. Craxton, "Highly Efficient Second-Harmonic Generation of Ultra-Intense Nd:Glass Laser Pulses," to be published in Optics Letters.

C. T. Cotton, "The Design of an All-Spherical, Three-Mirror, Off-Axis Telescope Objective," to be published in the OSA Proceedings of the International Optical Design Conference '94.

M. J. Cumbo, D. Fairhurst, S. D. Jacobs, and B. E. Puchebner, "Slurry Particle Size Evolution During the Polishing of Optical Glass," to be published in Applied Optics.

W. R. Donaldson and L. Mu, "Effect of Illumination Uniformity on GaAs Photoconductive Switches," to be published in the IEEE Journal of Quantum Electronics.

D. Fried, R. E. Gilena, J. D. B. Featherstone, and W. Seka, “The Nature of Light Scattering in Dental Enamel and Dentin at Visible and Near Infrared Wavelengths," to be published in Applied Optics.

R. E. Giacone, C. J. McKinstrie, and R. Betti, "Angular Dependence of Stimulated Brillouin Scattering in Homogeneous Plasma," to be published in Physics of Plasmas.

W. Gob, W. Lang, W. Kula, and R. Sobolewski, “Transport Properties and Superconducting Fluctuations in Oxygen Deficient Y-Ba-Cu-O Thin Films," to be published in Physica $C$. 
D. Gupta, W. R. Donaldson, and A. M. Kadin, "Energy Extraction from Superconducting Magnets Using Optically Activated $\mathrm{YBa}_{2} \mathrm{Cu}_{3} \mathrm{O}_{7-\mathrm{x}}$ Switches," to be published in Optically Activated Switching $I V$.

J. Hecht, D. Ofer, U. Alon, D. Shvarts, S. A. Orszag, and R. L. McCrory, "Three-Dimensional Simulations and Analysis of the Nonlinear Stage of the Rayleigh-Taylor Instability," to be published in Laser and Particle Beams.

P. A. Jaanimagi, R. C. Elton, B. L. Welch, Y. Leng, and H. R. Griem, "Extending X-Ray Streak Camera Operation to VUV Wavelengths," to be published in the Review of Scientific Instruments.

S. D. Jacobs, K. L. Marshall, and A. Schmid, "Liquid Crystals for Laser Applications," to be published in the CRC Handbook of Laser Science and Technology, Supplement 2: Optical Materials.

J. P. Knauer, R. L. Kremens, M. A. Russotto, and S. Tudman, "Using Cosmic Rays to Monitor Large Scintillator Arrays," to be published in the Review of Scientific Instruments.

E. M. Korenic, K. L. Marshall, and J. A. Maiolo, "Blending Polysiloxane 'Glass Resins' to Produce Optical Films with a Specific Refractive Index," to be published in Optics and Photonics News.

E. M. Korenic and S. D. Jacobs, "Cholesteric Liquid Crystal Inks and Paints," to be published in Optics and Photonics News.

W. Kula and R. Sobolewski, "Effect of Hydrogen Doping on Electrical Properties of Y-Ba-Cu-O Thin Films," to be published in Physica $C$.

W. Lang, G. Heine, W. Kula, and R. Sobolewski, "Study of Superconducting Fluctuations in $\mathrm{Bi}_{2} \mathrm{Sr}_{2} \mathrm{Ca}_{2} \mathrm{Cu}_{3} \mathrm{O}_{\mathrm{x}}$ Thin Films: Paraconductivity, Excess Hall Effect, and Magnetoconductivity," to be published in Physical Review B.

Y. Lin and T. J. Kessler, "Raman Scattering: A Four-Dimensional System," to be published in Applied Optics.

R. S. Marjoribanks, F. W. Budnik, H. Chen, and D. D. Meyerhofer, "Plasma Electron Temperature in Picosecond Laser Plasmas from Quasi-Steady Ratio of Isoelectronic Lines," to be published in Physical Review Letters.
K. L. Marshall, S. D. Jacobs, and J. E. Miller, "Mid-Infrared Modulation Using Field-Induced Scattering in Ferroelectric Liquid Crystals," to be published in Applied Optics.

F. J. Marshall and B. Yaakobi, "Quantitative Measurements with X-Ray Microscopes in Laser-Fusion Experiments," to be published in the Review of Scientific Instruments.

R. L. McCrory, "Progress Toward Ignition with Direct Drive," to be published in Concerning Major Systems in Science and Technology.

R. L. McCrory, J. M. Soures, C. P. Verdon, T. R. Boehly, D. K. Bradley, R. S. Craxton, J. A. Delettrez, R. Epstein, P. A. Jaanimagi, S. D. Jacobs, R. L. Keck, J. H. Kelly, T. J. Kessler, H. Kim, J. P. Knauer, R. L. Kremens, S. A. Kumpan, S. A. Letzring, F. J. Marshall, P. J. McKenty, S. F. B. Morse, A. Okishev, W. Seka, R. W. Short, M. D. Skeldon, S. Skupsky, M. Tracy, and B. Yaakobi, "Direct-Drive Laser Fusion Experimental Program at the University of Rochester Laboratory for Laser Energetics," to be published in the Proceedings of the Conference on Plasma Physics and Controlled Nuclear Fusion Research.

K. Mizuno, R. Bahr, R. S. Craxton, J. S. DeGroot, R. P. Drake, W. Seka, and B. Sleaford, "Direct Measurements of the Ion Acoustic Decay Instability in a Laser-Produced, Large Scale, Hot Plasma," to be published in Physical Review Letters.

L. Mu, W. Donaldson, J. C. Adams, and R. A. Falk, "Electromagnetic Wave Interaction with Laser-Induced Plasma in GaAs," to be published SPIE's Optically Activated Switching IV.

J. Z. Roach, A. Ninkov, S. W. Swales, and T. Morris, "Design and Evaluation of a Screen CCD Imaging System," to be published in Optical Engineering.

W. Seka, D. Fried, J. D. B. Featherstone, and S. F. Borzillary, "Light Deposition and Thermal Response in Dental Hard Tissue," to be published in the Journal of Dental Research.

H. Shi and S.-H. Chen, "Novel Glassy Nematic and Chiral Nematic Oligomers Derived from 1,3,5-Cyclohexanetricarboxylic and (1R,3S)-(+)-Camphoric Acids," to be published in Liquid Crystals.

H. Shi and S.-H. Chen, "Novel Glass-Forming Liquid Crystals. 2. Systems Containing High Optical Birefringence Moiety 
1-(Phenyl)-2-(6-Cyanonaphth-2-yl)Ethyne," to be published in Liquid Crystals.

M. D. Skeldon, A. Okishev, S. A. Letzring, W. R. Donaldson, and W. Seka, "Optically Activated Switches for the Generation of Complex Electrical Waveforms with Multigigahertz Bandwidth," to be published in SPIE's Optically Activated Switching $I V$.

D. J. Smith, J. F. Anzellotti, A. W. Schmid, S. Papernov, Z. R. Chrzan, and S. J. Van Kerkhove, "Damage Fluence at $1054 \mathrm{~nm}$ and $351 \mathrm{~nm}$ of Coatings Made with Hafnium Oxide Evaporated," to be published in the Proceedings of the XXVI Annual Symposium on Optical Materials for High Power Lasers.

R. Sobolewski and T. Y. Hsiang, "Progress in Ultrafast Superconducting Electronics," to be published in the Proceedings of the International Workshop on Superconductivity and Particle Detection. (invited)

C. J. Twomey, T. N. Blanton, K. L. Marshall, S. H. Chen, and S. D. Jacobs, "Some Dynamic Features of the Preparation of Liquid Crystalline Elastomers," to be published in Liquid Crystals.

C. P. Verdon and R. L. McCrory, "Direct-Drive Capsule Physics," to be published in the Proceedings of ECLIM '94.

C.-C. Wang, M. Currie, R. Sobolewski, and T. Y. Hsiang, "Subpicosecond Electrical Pulse Generation by Edge Illumination of Semiconductor Photoconductive Switches," to be published in Applied Physics Letters.
M. D. Wittman, R. Q. Gram, H. Kim, C. K. Immesoete, S. G. Noyes, and S. Scarantino, "Increased Retention Time for Hydrogen and Other Gases by Polymer Shells Using Optically Transparent Aluminum Layers," to be published in the Journal of Vacuum Science and Technology.

B. Yaakobi, Q. Su, F. J. Marshall, and R. Epstein, "Monochromatic Backlighting as a Laser-Fusion Diagnostic," to be published in the Journal of X-Ray Science Technology.

B. Yaakobi, D. Shvarts, F. J. Marshall, R. Epstein, and Q. Su, "Target Imaging and Backlighting Diagnosis," to be published in the Review of Scientific Instruments.

B. Yaakobi, R. Epstein, F. J. Marshall, D. K. Bradley, P. A. Jaanimagi, and Q. Su, "New Diagnostic Features in the Laser Implosion of Argon-Filled Targets," to be published in the Review of Scientific Instruments.

B. Yaakobi, F. J. Marshall, R. Epstein, and Q. Su, "New Diagnostic Features in the Laser Implosion of Argon-Filled Targets," to be published in Optics Communications.

X. Zhou, S. Alexandrou, and T. Y. Hsiang, "Monte Carlo Investigation of the Mechanism of Subpicosecond Pulse Generation by Nonuniform Gap Illumination," to be published in Applied Physics Letters.

J. D. Zuegel and W. Seka, "Direct Measurements of LowerLevel Lifetime in Nd:YLF," to be published in the Bulletin of the American Physical Society. 


\section{Conference Presentations}

The following presentations were made at $\mathrm{M}_{2} \mathrm{~S}$-HTSC IV, Grenoble, France, 5-9 July 1994:

W. Kula and R. Sobolewski, "Effect of Hydrogen Doping on Electrical Properties of Y-Ba-Cu-O Thin Films."

D. Wu, W. Kula, and R. Sobolewski, "Hopping Transport in YBa-Cu-O Films with Different Oxygen Content."

E. M. Korenic and S. D. Jacobs, "Selective Reflection Properties of Cholesteric Liquid Crystal Polymers," 3rd Annual Symposium on Materials Research, Rochester, NY, 16 August 1994.

The following presentations were made at the High Field Interactions and Short Wavelength Generation Topical Meeting, St. Malo, France, 21-25 August 1994:

D. D. Meyerhofer, C. I. Moore, and J. P. Knauer, "Forward Ponderomotive Acceleration of Electrons from the Focus of a High-Intensity Laser."
D. D. Meyerhofer, J. Chaloupka, and J. Peatross, "High-Order Harmonics Emitted from Low-Density Gas Targets."

J. L. Peatross, J. Chaloupka, and D. D. Meyerhofer, "HighOrder Harmonic Generation with an Annular Laser Beam."

C. P. Verdon and R. L. McCrory, "Direct-Drive Capsule Physics," ECLIM '94, Oxford, England, 19-23 September 1994.

R. L. McCrory, J. M. Soures, C. P. Verdon, T. R. Boehly, D. K. Bradley, R. S. Craxton, J. A. Delettrez, R. Epstein, P. A. Jaanimagi, S. D. Jacobs, R. L. Keck, J. H. Kelly, T. J. Kessler, H. Kim, J. P. Knauer, R. L. Kremens, S. A. Kumpan, S. A. Letzring, F. J. Marshall, P. J. McKenty, S. F. B. Morse, A. Okishev, W. Seka, R. W. Short, M. D. Skeldon, S. Skupsky, M. Tracy, and B. Yaakobi, "Direct-Drive Laser Fusion Experimental Program at the University of Rochester Laboratory for Laser Energetics," 15th International Conference on Plasma Physics and Controlled Nuclear Fusion Research, Madrid, Spain, 26 September-1 October 1994. 
